\title{
Artificial intelligence for imaging in immunotherapy
}

Citation for published version (APA):

Trebeschi, S. (2021). Artificial intelligence for imaging in immunotherapy. [Doctoral Thesis, Maastricht University]. Maastricht University. https://doi.org/10.26481/dis.20210322st

Document status and date:

Published: 01/01/2021

DOI:

10.26481/dis.20210322st

Document Version:

Publisher's PDF, also known as Version of record

\section{Please check the document version of this publication:}

- A submitted manuscript is the version of the article upon submission and before peer-review. There can be important differences between the submitted version and the official published version of record.

People interested in the research are advised to contact the author for the final version of the publication, or visit the DOI to the publisher's website.

- The final author version and the galley proof are versions of the publication after peer review.

- The final published version features the final layout of the paper including the volume, issue and page numbers.

Link to publication

\footnotetext{
General rights rights.

- You may freely distribute the URL identifying the publication in the public portal. please follow below link for the End User Agreement:

www.umlib.nl/taverne-license

Take down policy

If you believe that this document breaches copyright please contact us at:

repository@maastrichtuniversity.nl

providing details and we will investigate your claim.
}

Copyright and moral rights for the publications made accessible in the public portal are retained by the authors and/or other copyright owners and it is a condition of accessing publications that users recognise and abide by the legal requirements associated with these

- Users may download and print one copy of any publication from the public portal for the purpose of private study or research.

- You may not further distribute the material or use it for any profit-making activity or commercial gain

If the publication is distributed under the terms of Article $25 \mathrm{fa}$ of the Dutch Copyright Act, indicated by the "Taverne" license above, 
Doctoral thesis

\section{ARTIFICIAL INTELLIGENCE FOR IMAGING IN IMMUNOTHERAPY}

Stefano Trebeschi

2021 



\title{
ARTIFICIAL INTELLIGENCE FOR IMAGING IN IMMUNOTHERAPY
}

\author{
Dissertation
}

To obtain the degree of Doctor at Maastricht University, on the authority of the Rector Magnificus, Prof. Dr. R.M. Letschert, in accordance with the decision of the Board of Deans, to be defended in public on Monday 22nd of March, at 16.00 hours

by

Stefano Trebeschi 


\section{Promotor}

Prof. Regina G.H. Beets-Tan, MD PhD

Prof. Hugo Aerts, PhD

\section{Assessment Committee}

Prof. dr. Dirk de Ruysscher (chairman)

Prof. dr. Paul Hofman

Dr. Nicky Peters

Prof. dr. Paul Baas

The Netherlands Cancer Institute \& Leiden University

Prof. dr. Klaus Maier-Hein

German Cancer Research Center

Copyright (C) Stefano Trebeschi, Maastricht 2021.

All rights reserved. No part of this publication may be reproduced, stored in a retrieval system or transmitted in any form or by any means, electronic, mechanical, photocopying, recording or otherwise, without prior written permission of the author.

The publication of this thesis was financially supported by the Netherlands Cancer Institute and Maastricht University.

Cover $\quad$ Elmar van Zyl $\mid$ www.elmar.design

Production Gildeprint Enschede | www.gildeprint.nl

ISBN 978-94-6419-161-5 


\section{Contents}

1 Introduction 1

$\begin{array}{lll}2 & \text { Lesion response prediction to immunotherapy } & 7\end{array}$

2.1 Introduction . . . . . . . . . . . . . . . . . 9

2.2 Material and methods . . . . . . . . . . . . . . 10

2.3 Results . . . . . . . . . . . . . . . . 18

2.4 Discussion . . . . . . . . . . . . . . 22

2.5 Conclusions . . . . . . . . . . . . . . . . . . . 24

3 Lesion diagnosis of therapy-induced lung disease 27

3.1 Introduction . . . . . . . . . . . . . . . . . . . . . 29

3.2 Materials and methods . . . . . . . . . . . . . . 31

3.3 Results . . . . . . . . . . . . . . . . . 37

3.4 Discussion . . . . . . . . . . . . . . 42

3.5 Conclusions . . . . . . . . . . . . . . . 46

4 Prognostic value of chest imaging monitoring 53

4.1 Introduction . . . . . . . . . . . . . . . . . 55

4.2 Materials and methods . . . . . . . . . . . . . . 57

4.3 Results . . . . . . . . . . . . . . . 67

4.4 Discussion . . . . . . . . . . . . . . . 75

4.5 Conclusion . . . . . . . . . . . . . . . 79

5 Whole-body imaging-based prognostic monitoring 83

5.1 Introduction . . . . . . . . . . . . . . . 85

5.2 Materials and methods . . . . . . . . . . . . . 86

5.3 Statistical analysis . . . . . . . . . . . . . . . . 95

5.4 Results . . . . . . . . . . . . . . . . . 95

5.5 Discussion . . . . . . . . . . . . . . . . . . . 100

5.6 Conclusions . . . . . . . . . . . . . . . . . . . . . . . 104 
6 Prognostic response patterns in brain imaging 109

6.1 Introduction . . . . . . . . . . . . . . . . . . . 111

6.2 Materials and methods . . . . . . . . . . . . . . . 112

6.3 Results . . . . . . . . . . . . . . . . . . 118

6.4 Discussion . . . . . . . . . . . . . . . . . . . 122

6.5 Conclusions . . . . . . . . . . . . . . . . . 125

7 The future of artificial intelligence immunotherapy trials 131

7.1 Introduction . . . . . . . . . . . . . . . . . . . 133

7.2 AI in medical imaging . . . . . . . . . . . . . . . . 134

7.3 AI in pathology . . . . . . . . . . . . . . . . . . . . 140

7.4 AI in laboratory medicine . . . . . . . . . . . . . 143

7.5 Integrated artificial intelligence . . . . . . . . . . . . 145

7.6 The clinical trial of the future . . . . . . . . . . . 147

7.7 Conclusions . . . . . . . . . . . . . . . . . . . . . 151

8 Towards integrated healthcare 153

8.1 Introduction . . . . . . . . . . . . . . . . . 155

8.2 The promise of radiomics . . . . . . . . . . 156

8.3 Integrated systems in healthcare . . . . . . . . . . . 158

8.4 Conclusion . . . . . . . . . . . . . . . . . 159

9 Discussion 161

$\begin{array}{ll}\text { Bibliography } & 171\end{array}$

$\begin{array}{ll}\text { Valorisation } & 215\end{array}$

Summary 219

Acknowledgments 223

$\begin{array}{ll}\text { Published work } & 225\end{array}$

$\begin{array}{ll}\text { About the author } & 227\end{array}$ 


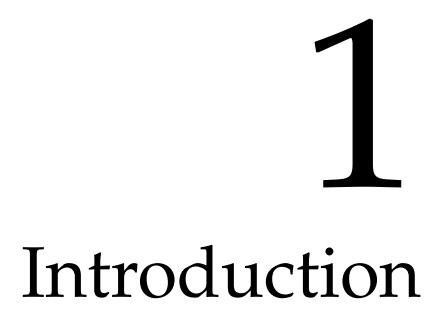



Immune checkpoint inhibitors have provided improved response rates and prolonged overall survival in advanced metastatic cancer patients. Several clinical trials have demonstrated more favourable outcomes compared to standard therapy, with the largest body of evidence being in melanoma [Lar+18; Web+15], non-small cell lung cancer [Vok+18; Bra+15; Car+17], renal cell carcinoma [Alb+20; Mot+20], and head and neck carcinoma [Sab+19; $\mathrm{Coc}+19 ; \mathrm{Pai}+19 ; \mathrm{Fer}+18]$. Additionally, the FDA has authorized the use of pembrolizumab in microsatellite instability high tumours (MSI-H) [Zha+19b; Luc+19] — i.e. tumours that had a genetic predisposition to mutation resulting from impaired DNA repair mechanisms [Ion+93; TBS93].

Despite the success of these immunotherapies, there is still a substantial number of patients who do not benefit from the treatment [Lar+18; Web+15; Vok+18; Bra+15; Alb+20; Mot+20; Fer+18]. Biomarkers able to identify these responders would ultimately improve treatment outcomes - both in terms of prolonged overall survival, as well as reduced therapy-induced toxicity - while simultaneously helping to contain the costs of these expensive anti-cancer therapies [GWA16; HCC19a; Voo+17; Tar+16].

Immunotherapy biomarker research has been focusing so far on biological markers, often extracted from invasive tumour-tissue biopsies. These include levels of infiltration of lymphocytes (i.e. white blood cells) in the tumour [Zit+17; He+17], or more general markers of inflammation [Aye+17], genetic mutations [McG+16], among others $[\mathrm{Ma}+16$; Men+15; Ker+15]. Their values, however, depend on the biopsied lesion, and on how generalizable they can be to the total tumour burden [Ram+20]. In advanced stage metastatic patients (the most likely target population for immunotherapy), multiple lesions across the body are likely to have developed distinctively [Cun+15; TS16] biological profiles, potentially as a result of their intrinsic microenvironment or the milieu of the organ where they are situated [Cun+15; TS16]. Due to the invasiveness of the biopsy procedure [Ove+13] in an already fragile patient cohort of advanced-stage cancer, it is not always feasible to biopsy multiple locations to average out inter-lesional het- 
erogeneity, nor is it possible to perform multiple biopsies of the same location over time to evaluate response to treatment.

Different alternatives are being explored to overcome these limitations, including whole-body imaging. Routine radiological imaging has already emerged as a fundamental tool in the clinics for detection, characterisation, and monitoring of disease [Hri11]. It is non-invasive, and it is broad - i.e. able to capture whole-body information in one single measurement. These characteristics led imaging to become the default tool for the diagnosis [Mek+18] and follow-up of advanced-stage cancer patients receiving immunotherapy [Sey+17a; The+00], playing an irreplaceable role in treatment planning [Ner+20]. Currently, the read-out and interpretation of radiological imaging is done by a medical professional via visual assessment [Sey+17a; The+00]. They report the presence and location of cancer lesions, their extent or size, their evolution in time [Sey+17a; The+00; Sch+16; Eis+09; Org+79; $\mathrm{Hay}+77]$, and the presence of other clinically-relevant non-cancer conditions [Mek+18]. The most common tool used in the clinics is the Response Evaluation Criteria in Solid Tumour (RECIST) [Sey+17a; Sch+16]. This prescribes the follow-up of the cumulative in-plane diameter of maximum 5 target lesions. Depending mainly on the changes in the cumulative diameter, the response is classified as complete or partial, stable, or progressive disease; with minor changes made to accomodate for patients receiving immunotherapy. While this method is timeefficient (required aspect in the busy radiological practice), it lacks to address any prognostic factor that is not related to total tumor growth or shrinkage - among other limitations [VS13].

There is a growing body of evidence that more clinically-valuable information can be extracted from radiological imaging [Aer+14; Hos+18; $\mathrm{Bi}+19 \mathrm{a}$; $\mathrm{AH} 16$ ]. It is hypothesized that underlying biological processes (some of which regulate response to therapy or survival) are reflected in imaging patterns on radiological scans [Aer+14; Aer16; Par+15; OCo+17]. Computational methods, able to analyze and identify patterns in high dimensional imaging data, can serve this purpose. Two main techniques are relevant to this end: radiomics 
[Aer+14] and artificial intelligence [Hos+18]. Radiomics entails all the computer algorithms for data characterisation in medical imaging that aim to extract imaging features, each feature describing anatomical or functional characteristics of, e.g. a cancer lesion [Aer+14; Par+15; GKH16; Gri+17a]. The main advantage relies on the usage of computer algorithms, which allow for the disentanglement and identification of imaging (pixel) patterns that would not be able to be assessed by the naked eye. Once imaging patterns have been extracted, these need to be linked to a clinically-relevant variable, e.g. the outcome of the treatment. Artificial intelligence (AI) is used to this end [Hos+18; $\mathrm{Bi}+19 \mathrm{a}]$. $\mathrm{AI}$ is the collection of all the computational methods which, through a learning process on plain or processed data, are able to decipher patterns and link variables together, ultimately extrapolating knowledge for "reasoning", or inference [RN03].

This thesis aims to develop and assess the value of AI-based radiomics methods on imaging data as clinical support systems for response evaluation, prediction and prognostication of advanced-stage cancer patients receiving immune checkpoint inhibitors.

This was formulated in two main research questions:

- Can AI-radiomics methods on radiological imaging detect and extract quantitative imaging patterns of single lesions that are related to lesion biology and lesion response to checkpoint inhibitors?

- Can AI-radiomics methods be developed to decipher prognostic morphological patterns at whole-body level in advanced stage metastatic patients receiving checkpoint inhibitors?

The outline of this thesis follows the research questions. It begins with the question of imaging features of single lesions for lesion assessment and response prediction. Chapter 2 evaluates predictive lesionlevel radiomic features for the prediction of individual lesion response. Chapter 3 evaluates the diagnostic performance of radiomic features 
for the diagnostic profiling of lung lesions, and gives an overview on how single lesion profiling can be correlated with long term outcome. The research question is then amplified to encompass the development of whole-body AI-based methods for the prognostication of metastatic patients receiving immunotherapy, overcoming in this manner the limitations of single lesion analysis. Chapter 4 introduces the concept of prognostication through AI-based image monitoring of chest imaging in NSCLC. Chapter 5 expands to thoracoabdominal imaging in urothelial cancer patients and formalizes it as the Prognostic AI-Monitor (PAM). Chapter 6 investigates PAM in brain metastasis, extending our findings to the whole body. This thesis concludes with Chapters 7 and 8, stating the future developments of artificial intelligence research in the clinics, and in cancer immunotherapy trials. 


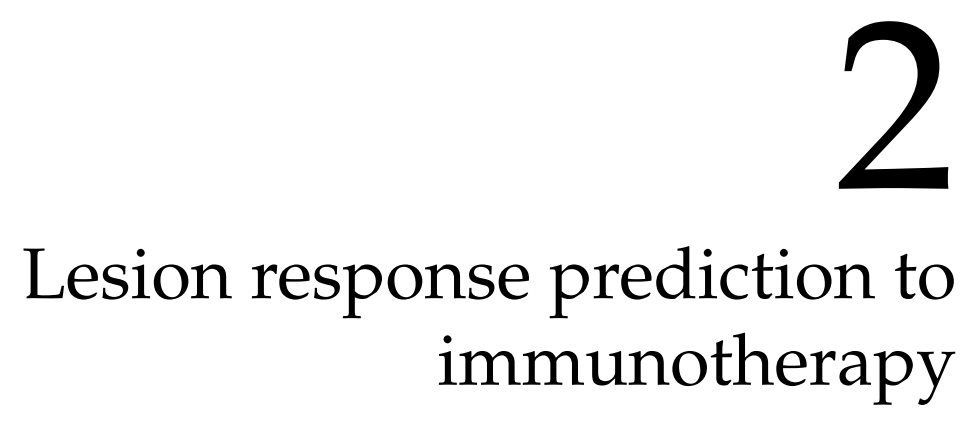

Stefano Trebeschi et al. "Predicting response to cancer immunotherapy using noninvasive radiomic biomarkers". In: Ann. Oncol. 30.6 (2019), pp. 998-1004. 


\section{Abstract}

Introduction Immunotherapy is regarded one of the major breakthroughs in cancer treatment. Despite its success, only a subset of patients responds - urging the quest for predictive biomarkers. We hypothesize that Artificial Intelligence (AI) algorithms can automatically quantify radiographic characteristics that are related to and may therefore act as non-invasive radiomic biomarkers for immunotherapy response.

Patients and Methods In this study, we analyzed 1055 primary and metastatic lesions from 203 patients with advanced melanoma and non-small cell lung cancer (NSCLC) undergoing anti-PD1 therapy. We performed a AI-based characterization of each lesion on the pretreatment contrast-enhanced CT imaging data to develop and validate a non-invasive machine learning biomarker capable of distinguishing between immunotherapy responding and non-responding. To define the biological basis of the radiographic biomarker, we performed gene-set enrichment analysis in an independent dataset of 262 NSCLC patients.

Results The biomarker reached significant performance on NSCLC lesions (up to 0.83 AUC, $p<0.001$ ) and borderline significant for melanoma lymph nodes (0.64 AUC, $p=0.05)$. Combining these lesion-wide predictions on a patient level, immunotherapy response could be predicted with an AUC of up to 0.76 for both cancer types $(p<0.001)$, resulting in a one year survival difference of $24 \%(p=0.02)$. We found highly significant associations with pathways involved in mitosis, indicating a relationship between increased proliferative potential and preferential response to immunotherapy.

Conclusions These results indicate that radiographic characteristics of lesions on standard-of-care imaging may function as non-invasive biomarkers for response to immunotherapy, and may show utility for improved patient stratification in both neoadjuvant and palliative settings. 


\subsection{Introduction}

Cancer immunotherapy has made promising strides as a result of improved understanding of biological interactions between tumor cells and the immune system. Both the EMA and the FDA have approved anti-PD1 antibodies to treat melanoma or non-small cell lung cancer (NSCLC) patients with unresectable or metastatic disease, which progressed under platinum-based chemotherapy or display high expression of PD-L1 [U Sb; U Sa; Eurb; Eura] — with overall response rates of $44 \%$ and $32 \%$ in first and second line in melanoma [Wol+15; Web+15] and $19 \%$ in second line in lung cancer [Bor+15; Bra+15; Her+16]. Unlike traditional cancer treatment, anti-PD1 antibodies potentiate the anti-tumor immune response.

Despite their remarkable success, clinical benefit remains limited to only a subset of patients [Hod+10]. As immunotherapy is expensive and could bring toxicity, there is a need to stratify patients according to likely benefit prior to therapy. Different biomarkers have been investigated with variable success, such as levels of PD-L1 [Ma+16; Men+15; Ker+15], presence of tumor infiltrating lymphocytes [Zit+17; $\mathrm{He}+17]$, genetic mutations [McG+16; Riz+15; Hel+18a], and inflammatory cytokines [Aye+17].

Recent emergence of quantitative imaging biomarkers provide promising opportunities. Unlike traditional biopsy-based assays that represent only a sample of the tumor, images reflect the entire tumor burden, providing information on each cancer lesion with a single noninvasive examination. This is of particular importance in immunotherapy, where different lesions can have different microenvironments potentially leading to heterogeneous response patterns [Whi08]. Previously, radiolabeled anti-PD1 antibodies were used to visualize specific immunological expressions [Wu09].

Computational imaging approaches originating from Artificial Intelligence (AI) have achieved impressive successes in automatically quantifying radiographic characteristics of tumors [Hos+18]. 
AI-based characterization on radiology is referred to as "radiomics" and can provide more detailed characterization than possible by eye [Hos+18; Aer+14; AH16]. Radiomics-based biomarkers have shown success in different tumor types [Cor+16; Kir+17; Fav+17; Par+15; $\mathrm{Kic}+16$; Pra+16; $\mathrm{Li}+16$; ; but to the best of our knowledge, there is no evidence yet in immunotherapy. Tumor morphology, visualized on imaging, is likely influenced by several aspects of tumor biology. We hypothesize that a set of morphological characteristics, quantified by radiomics, are related to and may therefore act as predictive markers.

In this study, we analyzed all visible cancer lesions to evaluate the potential predictive value of CT-derived radiomic biomarkers in metastatic NSCLC and melanoma patients receiving immunotherapy. A biologic evaluation was performed in an independent validation set of surgical NSCLC patients with imaging and gene-expression data.

\subsection{Material and methods}

\subsubsection{Immunotherapy dataset}

Patients with metastatic melanoma or NSCLC receiving $3 \mathrm{mg} / \mathrm{kg} / 2$ weeks of anti-PD1 at the Netherlands Cancer Institute (NKI) between 2014 and 2016 were retrospectively analyzed. Contrast-enhanced computed tomography (CE-CT) scans were acquired before (baseline) and around 12 weeks after start of treatment (follow-up). The study protocol was approved by the Medical Ethics Committee and Board of Directors of the NKI and informed consent was waived.

Image acquisition protocol. The CT scans were performed by either covering the chest $(n=86)$ or covering the chest and abdomen $(n=117)$ using multi-slice CT equipment (Toshiba Aquilion CX, Minato, Tokyo, Japan; Siemens Somatom Sensation Open, Erlangen, Germany) with 
a tube voltage of $120 \mathrm{kVp}$, slice thickness of $1 \mathrm{~mm}$, and in-plane resolution of $0.75 \times 0.75 \mathrm{~mm}$. The bolus injection was performed at 3 $\mathrm{ml} / \mathrm{s}$ (Omnipaque 300, GE Healthcare, Chicago, Illinois, US) not prewarmed, with a total amount based on the patient weight $+40 \mathrm{cc}$ (minimum of $90 \mathrm{cc}$ and maximum of $130 \mathrm{cc}$ ) followed by a saline flush of $30 \mathrm{cc}$. The chest CT examinations were performed 40 seconds after contrast injection, whereas the chest and abdomen examinations were performed at 70 seconds.

\subsubsection{Genomics dataset}

To provide biological validation, we evaluated an independent, dataset of surgical NSCLC patients between 2006 and 2009 treated at the H. Lee Moffitt Cancer Center. Pre-surgical CE-CT (within 60 days of diagnosis) and gene expression data was available for 262 patients. The University of South Florida IRB approved and waived informed consent (IRB\#16069); in accordance with HIPAA (more information in the original publication [Gro+17]).

Image acquisition protocol. Contrast-enhanced CT scans were acquired 60 days within diagnosis, as part of the Thoracic Oncology Program protocol, of the L. Lee Moffitt Cancer Center (Tampa, Florida, USA). Gene expression of 60,607 probes was measured on a custom Rosetta/Merk Affymetrix 2.0 microarray chipset (HuRSTA2a520709.CDF, GEO accession number GPL15048) by the Moffitt. The University of South Florida IRB institutional review board approved and waived the informed consent requirement (IRB\#16069); data were collected and handled in accordance with the Health Insurance Portability and Accountability Act. Informed consent for gene expression collection was written and oral. For acquisition of imaging and clinical data USF IRB approved protocol (IRB\#108426) provided a waiver of informed consent. 


\subsubsection{Chemotherapy dataset}

To study the specificity of the radiomic biomarker for immunotherapeutic response prediction, we retrospectively collected a cohort of 39 patients with stage IV NSCLC treated with neoadjuvant chemoradiotherapy at NKI between 2012 and 2016 (IRBd18079).

Image acquisition protocol. The CT scans were performed covering the chest and abdomen $(n=39)$ using multi-slice CT equipment (Toshiba Aquilion CX, Minato, Tokyo, Japan; Siemens Somatom Sensation Open, Erlangen, Germany) with a tube voltage of $120 \mathrm{kVp}$, slice thickness of $1 \mathrm{~mm}$, and in-plane resolution of $0.75 \times 0.75 \mathrm{~mm}$. Specific of the scanning protocols were identical to the immunotherapy dataset.

\subsubsection{Imaging data and lesion segmentations}

Experienced readers manually delineated lesions on baseline and follow-up scans. Target lesions were defined as any tumor that was well-demarcated on both baseline and follow-up with diameter $\geq 5$ $\mathrm{mm}$. The inclusion criteria were: availability of CE-CT BL and FU and, presence of measurable target lesions at baseline. Measurable lesions were defined as any tumor lesions (primary or metastatic lesions) whose entire border could be identified on both BL and FU scans, as our radiomic feature extraction pipeline requires segmented region of interest to extract features Lesions that disappeared in the FU were flagged as complete response. Lesions that could not be accurately discriminated from surrounding tissues (e.g. lung nodule within atelectasis), with ill-defined borders (e.g. lung lesions adjacent to atelectasis) and lesions which could not be tracked down from other adjacent tumour lesions at baseline or follow-up CTs (e.g. confluent metastases) were not delineated and excluded. Lesions poorly visualized because of the presence of imaging artefacts (e.g. scattering, motion or breathing artefacts) were excluded as well. Examples are shown in Figures 2.1a-b. 


\subsubsection{Response kinetics}

To assess the effects of mixed response, we performed a lesion-perlesion assessment of relative change in diameter between baseline and follow-up, using RECIST criteria. Furthermore, in patients with $>1$ lesion, we classified response patterns on a patient basis as mixed for patients presenting both responding and progressive lesions and uniform for patients presenting only responding or progressive lesions (irrespective of stable lesions). This setup allows for the characterization of overall tumor burden.

\subsubsection{Radiographic differences between responding and progressive lesions}

To generate radiomic sequences for each lesion at baseline, a set of radiomic features was defined [G217] (see Figure 2.1e). Radiomic features of responding responding and progressive lesions were directly compared to identify differences. To reduce redundancy, ten complementary features were selected using unsupervised feature selection [Yin08]. Statistical significance was assessed using generalized mixedeffect models - controlling for patient, tumor type and organ. False discovery rate (FDR) was at $10 \%$ to correct for multiple comparisons.

\subsubsection{Radiomic biomarkers to predict immunotherapy response of cancer lesions}

To assess the performance of the radiomic biomarker, we developed a machine learning model [Cox58; B201; Ols+16]. We trained the model on all lesions (i.e. progressive, stable and responding) to discern progressive disease. The dataset was divided into training, tuning, and testing sets based on patient identifiers. The training set was used to model data distributions. The tuning set was used during training to control for overfitting. The test set was used for independent evaluation (see Figure 2.1f). Mann-Whitney-U test was used for statistical 
testing of AUC curves, one-sided McNeils test was used to test if the radiomic biomarker was outperforming volume and maximum diameter, and log-rank test was used for statistical testing of survival performance.

To test for radiomic association with molecular pathways, Spearman's rank correlation coefficient was used. Pathways were then ranked by $-\log 10(p)$, where $p$ is the correlation $p$-value, and put into a preranked gene set enrichment analysis (GSEA) algorithm [Sub+05] version 2.0.14 on the C2 collection version Molecular Signature Database (MSigDB)[Lib+11].

Feature extraction. To reduce the influence of outlier intensity values in the image, the volume was clipped between -1000 HU and $3000 \mathrm{HU}$. Radiomic features were extracted from original images as well as from different image transformations including five Laplacian of Gaussian filters $(\sigma=1.0,2.5,5.0,7.5,10.0 \mathrm{~mm})$, eight wavelets decompositions, and four non-linearities (exponential, square, square root and logarithm). We also repeated the extraction over three different scales, each defined by a set of radiomic parameters: (1) a fine scale with 1 $\mathrm{mm}$ isotropic resolution and $1 \mathrm{HU}$ bin width, (2) a medium scale with $3 \mathrm{~mm}$ isotropic resolution and bin width of $5 \mathrm{HU}$ and (3) coarse scale with $5 \mathrm{~mm}$ isotropic resolution and bin width of $25 \mathrm{HU}$. In this way, the algorithm can choose the best radiomic extraction parameters and/or their combination. Features which resulted in invalid values for more than one lesion were dropped.

Dataset preparation. The entire dataset was divided into train, validation and test set based on patient identification numbers (pid). Patients whose pid was divisible by three were assigned to the train set, those whose (pid - 1) was divisible by three were assigned to the validation set, and those whose (pid - 2) was divisible by three were assigned to the test set.

Classifier pool. The first group is composed by three linear classifiers based on logistic regression (LR) models [Cox58], each differentiated by a different feature selection method: (1) unsupervised result- 
ing from PCA, (2) supervised resulting from wrapper feature selection (WFS), or (3) no feature selection. Similarly, we defined a second group of non-linear classifiers based on random forests (RF) [B201]. Finally, we generated two additional classifiers via genetic evolution (GEN-1 and GEN-2). Each classifier was trained using 2-fold cross validation and optimized via sequential model based optimization.

Training strategy. Each classifier is trained on the training set using a 2 -fold cross validation procedure. To prevent the model from learning to recognize patients rather than the actual lesion-wise classification task, we enforced cross validation at a patient level, avoiding the distribution of lesions of the same patient across different folds. Once trained, the model is evaluated in on the test set to check for under- or overfitting, and model selection.

Classifier optimization. Each classifier comes with a set of tunable parameters, i.e. hyperparameters. We made use of a machine learning procedure, a.k.a. sequential model based optimization (SMBO), to tune the hyperparameters of each classifier. SMBO procedure is an iterative procedure, where at each iteration the performance is modelled as a function $\mathrm{f}$ of the hyperparameters. The search of the optimal hyperparameters is achieved via optimization of a criterion on $f$. We chose the commonly used Expected Improvement (EI). Parzen estimators were used to approximate the function $f$.

Hyperparameter space. Logistic regressions had only one tunable hyperparameter representing the weight of the $L_{2}$ regularization coefficient. Random forests had four hyperparameters: the max depth of the trees $(d)$, the minimum number of samples in each leaf $(m L)$, the minimum number of samples $(m S)$ and minimum Gini impurity in each split $(G)$. The hyperparameters of the genetic classifiers depend on the specific search result. Finally, wrapper feature selectors had one hyperparameter $k$, indicating the number of top-performant features selected. For each classifier, we selected the set of hyperparameters resulting in the highest AUC. 
Model selection. Once completed, the optimization procedure results in a set of eight trained classifiers. We selected the final classifier by comparing their performance on the validation set. The classifier that achieved the highest AUC score was selected as candidate solution. All algorithms, except for wrapper random forests and the second genetic evolution classifier, reported a certain degree of overfitting quantified by a lower accuracy on the validation set w.r.t the one reported on the training set. During training, all algorithms perform similarly between the two folds of cross validation, except second genetic evolution classifier which showed higher variance. Our choice of using wrapper random forests as candidate classifier was motivated by the fact that this configuration reached the highest performance with the least amount of overfitting. 

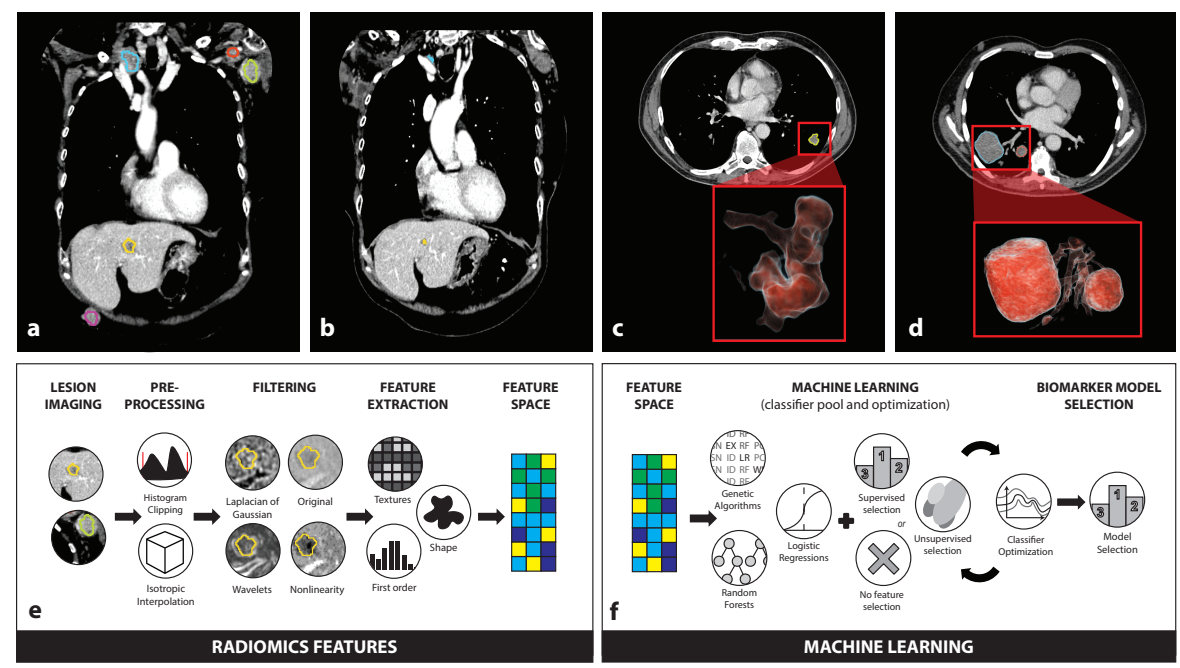

Figure 2.1: (a) Baseline contrast-enhanced CT scan of melanoma patient presenting with metastases in the liver and lymph nodes in the axilla and subclavicular area (b) Follow-up scan of the same patient showing complete response in the axillary region and partial response of the lesions in the liver and neck (c) Baseline CT scan of a NSCLC patient presenting lesion in the left lung, that showed progression at a later FU CT (not shown) (d) Baseline CT scan of a melanoma patient presenting lesions in the right lung, that showed response at a later FU CT (not shown) (e) Schematic representation of the radiomics feature extraction process (f) Schematic of the machine learning process. 


\subsection{Results}

\subsubsection{Immunotherapy response kinetics}

To assess immunotherapy response kinetics, 203 (123 NSCLC, 80 melanoma) patients were analyzed with a total of 1055 target lesions. Lesions were similarly distributed between NSCLC ( $n=572,54 \%)$ and melanoma $(n=483,46 \%)$. The most common lesion sites were lung $(\mathrm{n}=359,34 \%)$, lymph nodes $(\mathrm{n}=312,30 \%)$, and liver $(\mathrm{n}=212,20 \%)$. Most lesions ( $\mathrm{n}=746$ vs 309 , chi-square-test $p<0.01$ ) showed either stable $(n=395)$ or partial response $(n=351)$.

Melanoma lesions showed better overall response than NSCLC $(40 \%$ vs $27 \%$ responding, $p<0.01 ; 23 \%$ vs $34 \%$ progression, $p<0.01$ ). This trend was more evident for lung lesions, where we observed progression in NSCLC ( $39 \%$ vs $14 \%, p<0.01)$ and response in melanoma $(48 \%$ vs $26 \%, p<0.01)$. Hepatic melanoma lesions showed response in comparison with NSCLC ( $22 \%$ vs $36 \%, p=0.04)$. Examples are shown in Figures 2.1c-d.

Comparing per-patient response patterns in both cancer types, we observed that $23 \%(n=47)$ showed uniform response, $27 \%(n=55)$ uniform progression, and $22 \%(n=45)$ mixed response. The remaining $28 \%$ $(n=56)$ of the patients did not have multiple target lesions or presented only stable lesions. Significantly higher survival rates were seen in uniform response (log-rank test, $p<0.01$ ). This was evident in melanoma (log-rank-test, $p<0.01$ ), while in NSCLC, despite similar trends, did not reach significance $(p=0.08)$. Per-patient response kinetics are shown in Figure 2.2a. Kaplan-Meier curves are shown in Figures 2.2b-d.

\subsubsection{Radiographic differences between responding and progressive lesions}

To investigate radiographic differences between responding and progressing lesions, we compared their radiomic features (see Table 2.1). Among the most common locations (lung, lymph nodes, 


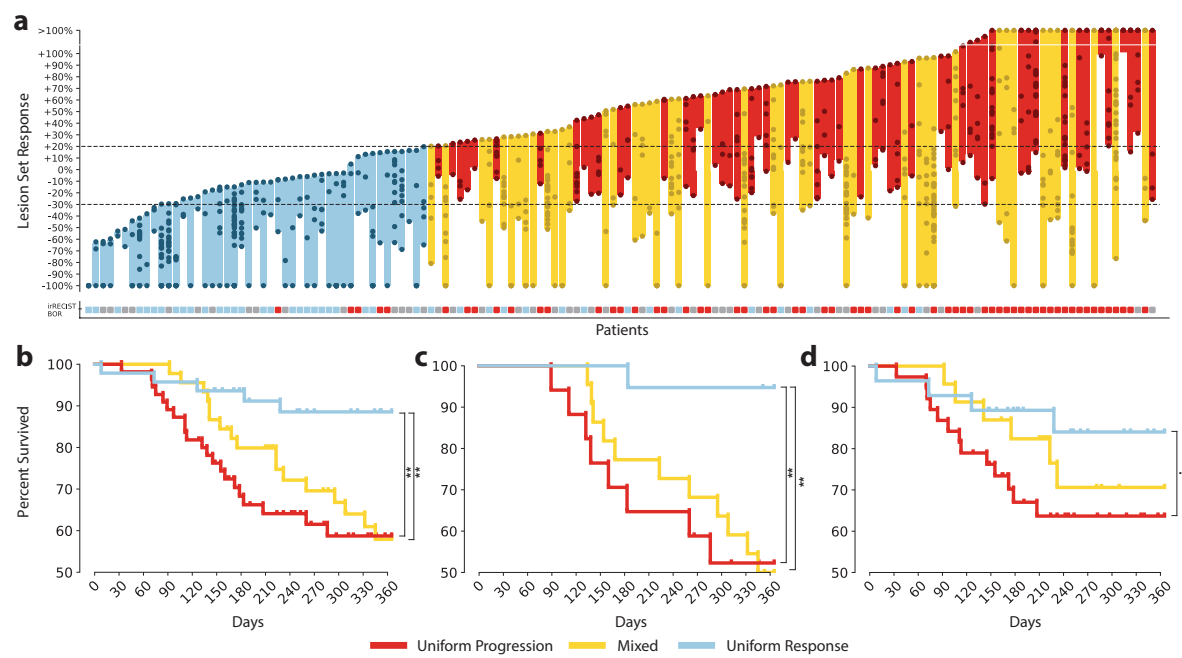

Figure 2.2: (a) Response kinetics curve depicting individual lesion responses (as dots) on a patient-to-patient basis (b) 1 year survival plot for all analyzed patients, (c) for melanoma patients only, (d) for NSCLC patients only.

liver and adrenal gland), responding lesions presented higher levels of irregular patterns (Wavelet.HLH_GLSZM_ZoneEntropy, Kenward-Roger-test $p=0.007)$ with more compact, spherical profiles (SurfaceVolumeRatio, $p=0.01$ ). Subanalysis on location revealed increased values of morphological heterogeneity in hepatic, nodal, and splenic lesions associated with response $(p<0.02)$. Of the most common NSCLC lesions, similar trends for morphological heterogeneity were seen at organ level in pulmonary and hepatic lesions, as well as lymph nodes also characterized by the presence of hypodense regions $(p=0.007)$. No significance was observed in primary NSCLC tumors. Among most common melanoma lesions greater morphological heterogeneity showed association with response (GLCM'DifferenceEntropy, $p=0.006$ ). Similar trends for morphological heterogeneity were seen but lower sample numbers did not allow to pass the patient correction. 


\subsubsection{Radiomic biomarker to predict immunotherapy responding and stable lesions}

To assess the performance of radiomics to rule out progression, we used machine learning to develop a single radiomic biomarker with 133 patients in the discovery set and 70 patients in test (see Table 2.2). A random forest with wrapper feature selection was used to develop radiomic biomarkers based on the performance in the discovery set and were validated on the independent test set.

In NSCLC, radiomic biomarker from pulmonary (0.83 AUC, MannWhitney-U-test $p<0.001)$ and nodal metastases (0.78 AUC, $p<0.001)$ showed significant performance. Satisfactory performance was observed in NSCLC primary tumors (0.79 AUC, $p=0.05)$, hepatic $(0.75$ AUC, $p=0.13$ ) and adrenal lesions ( $0.70 \mathrm{AUC}, p=0.18$ ) but did not reach significance mostly due to the low number of samples. The model performed poorly on both pulmonary and hepatic melanoma lesions (0.55 AUC). Despite these results, a trend toward significance is shown in nodal metastases (0.64 AUC, $p=0.05$ ) (see Figure 2.3a). Evaluation of the radiomic biomarker on all 303 lesions within the test dataset resulted in significant predictive performance ( 0.66 AUC, $p<0.01$; see Table 2.2).

By combining predictions made on individual lesions, it is possible to do a pre-treatment patient-wise prediction of immunotherapy response (see Methods). Significant performances were observed to predict OS for both tumour types (0.76 AUC for all patients, $p<0.01 ; 0.76$ AUC for NSCLC patients, $p<0.01 ; 0.77$ AUC for melanoma patients, $p<0.01$; see Figure 2.3b), with a significant survival difference at 1-year of $25 \%$ ( $77 \%$ vs $52 \%$, log-rank-test, $p=0.02$; see Figure $2.3 \mathrm{c})$. Interestingly, in melanoma patients, we observed significant performance to predict OS and response, despite the lower performance on a lesion level.

This radiomic immunotherapy response biomarker could not significant predict overall survival in patients treated with 
neoadjuvant chemoradiotherapy $(p=0.07)$, nor in terms of overall patient response $(\mathrm{AUC}=0.63 ; p=0.09)$. In terms of lesion response, the biomarker was inversely correlated to response of lung lesions in non-immunotherapy patients $(\mathrm{n}=61, \mathrm{AUC}=0.70, p=0.04)$, but did not show any significant predictive value in the remaining nodal $(\mathrm{n}=61$, AUC $=0.59, p=0.24)$ and liver lesions $(\mathrm{n}=12, \mathrm{AUC}=0.65, p=0.29)$.
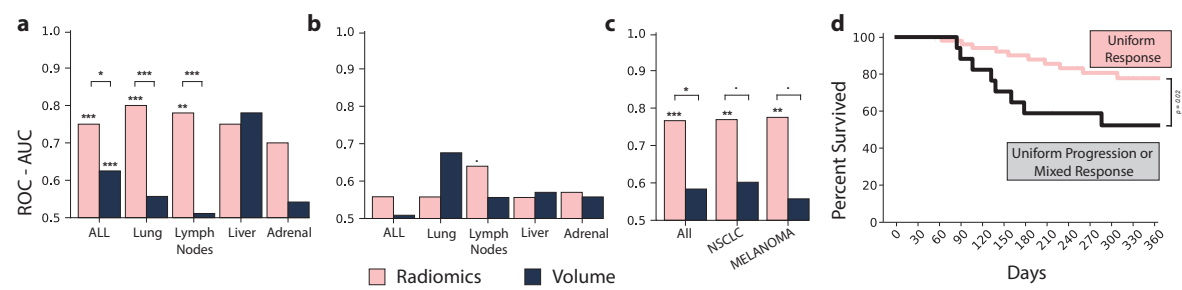

Figure 2.3: Performance of the selected classifier on the independent test set for (a) NSCLC lesions and (b) melanoma lesions. (c) Patient level response at first follow-up, and (d) Prognostic performance of the imaging biomarker on a patient level.

\subsubsection{Biological validation of the radiomic biomarker}

To evaluate the biological basis of the radiomic biomarker, we evaluated it in an independent dataset of 262 NSCLC patients with matched array-based gene expression data [Gro+17]. Through ranked gene set enrichment analysis, we found that the top gene-sets showing significant association with the radiomic biomarker were involved in cell cycle progression and mitosis. This indicates that a link between high tumor proliferation and improved response to immunotherapy may exist, and provides rationale for early-application immunotherapy as a therapeutic option for aggressive rapidly-expanding cancers. 


\subsection{Discussion}

Our aim was to evaluate radiomics-based models and their potential to predict treatment response in metastatic melanoma and NSCLC patients receiving anti-PD-1 antibodies.

We found that lesions with more heterogeneous morphological profiles with non-uniform density patterns and compact borders are more likely to respond to immunotherapy - irrespective of organ and/or cancer type. Higher levels of SurfaceVolumeRatio in nonresponding lesions in both cancers suggest that more compact and spherical profiles are associated with better response.

Based on these results, it would be prudent to point out that morphological heterogeneity does not necessarily correspond to genetic heterogeneity: infiltration, inflammation, neovascularization, and necrosis could also be associated with morphological irregularities. Assuming that a well-vascularized monoclonal tumour growing in the absence of an immune system would expand uniformly in all directions, any deviation could suggest a fault of one of aforementioned characteristics. If we were to relax one of these conditions, e.g. by adding an immune system, we would observe infiltration and inflammatory microenvironment $[\mathrm{McG}+16]$ affecting the tumor morphology - now comprising more than solely tumor cells. Irregular vascularization might cause non-homogeneous growth patterns while hampering Tcell infiltration [Hua+13]. The role of the other compartments need to be taken into account in order to explain the overall tumor growth.

Overall results of machine learning model show good predictive performance for NSCLC metastases. In melanoma the same model performed poorly. Besides the smaller melanoma cohort, the heterogeneous therapeutic backgrounds likely played a role in the morphological characterisation. While NSCLC patients received chemotherapy as first-line, melanoma patients received a variety of different treatments prior to immunotherapy. This could potentially have led to standardization of defined genetic profiles and tumour microenvironments in 
NSCLC [GM12; Aer+14; Gro+17; Rio+17]. In melanoma patients the diversity of therapeutic backgrounds might have induced different genetic profiles and microenvironments. Despite the lower performance on individual melanoma lesions, we still see a correlation with response and overall survival at a patient level, suggesting a relationship between individual lesion response and overall tumor burden.

GSEA on an external cohort revealed associations of the radiomic biomarker to proliferative potential in NSCLC, suggesting that highly proliferative tumors may show preferential response to immunotherapy. While standard of care for patients with aggressive cancer showing rapid expansion is platinum-doublet chemotherapy, these results provides the biological rationale for previous work demonstrating why combination therapy is a viable option in first-line metastatic settings, [Gan+18b].

We designed the study using a lesion-based approach, reflecting the metastatic condition characterizing patients receiving immunotherapy. This enabled us to investigate lesions individually while avoiding the issue of mixed response. Whenever possible, we limited selection biases and tried to avoid overfitting. Further validation in larger cohorts is warranted.

As imaging can provide information of the total tumor burden which allows the analysis of each lesion individually, its value lies complementary to currently known biomarkers (limited to single lesion samples). Despite the correlations found to overall patient survival and molecular pathways, further studies are needed to investigate the interaction between single (or clusters of) lesions, tumor biology and clinical status. Only a multidisciplinary aimed to integrate data from different disciplines can create a fully integrated solution that can be implemented into the clinical workflow. 


\subsection{Conclusions}

Our findings suggest associations between radiomics characteristics and immunotherapy response showing consistent trends across cancer types and anatomical location. Lesions that are more likely to respond to immunotherapy typically present with more heterogeneous morphological profiles with non-uniform density patterns and compact borders. Moreover, we provide a predictive machine learning model that could be used within the context of lesion response to treatment, patient treatment response, and response pattern characterization. Furthermore, we evaluated the biological basis of the proposed biomarker and found to be correlated with cell proliferative potential. Motivated by the results and the wide availability of routine clinical CT scans for cancer immunotherapy patients, we aim to expand this research further to the design of clinically applicable automatic computer models that could support the oncological decision-making process. 


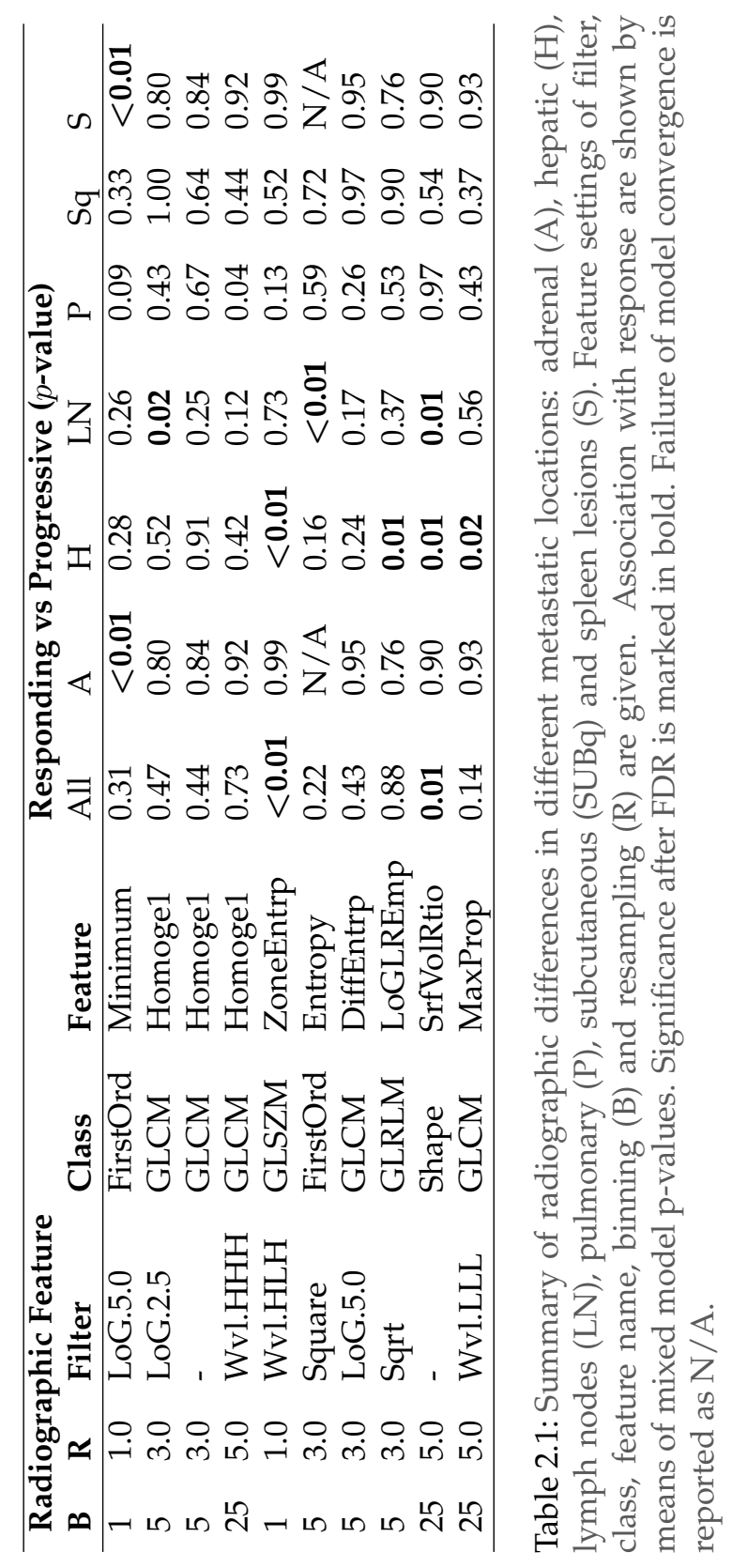




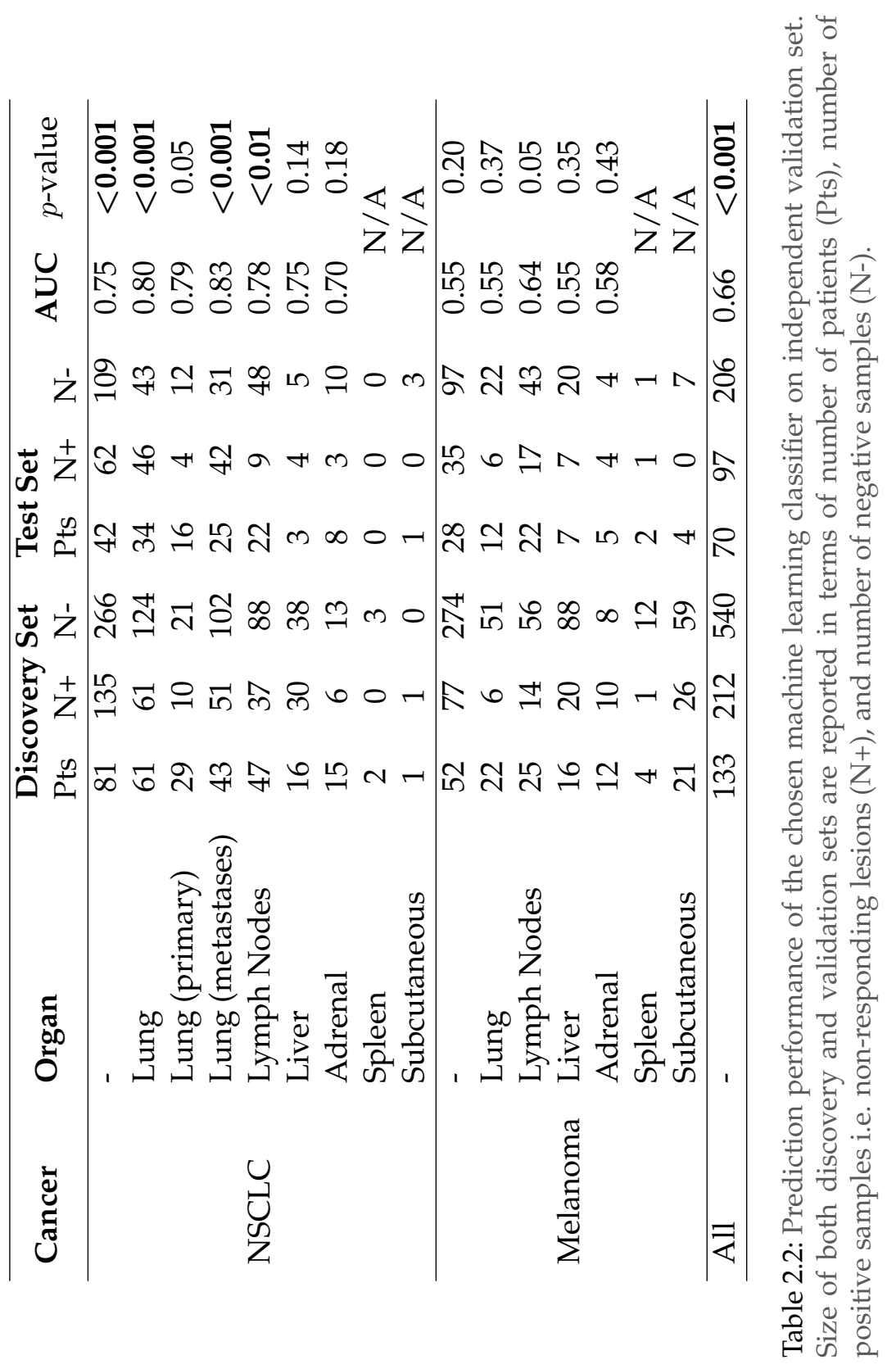




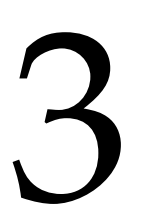

\section{Lesion diagnosis of therapy-induced lung disease}

Stefano Trebeschi et al. "Deep learning distinguishing pulmonary progression from pulmonary sarcoid-like lesions in immunotherapytreated melanoma patients". In: British Journal of Cancer, accepted for publication (2020). 


\section{Abstract}

Background. Immunotherapy is being used in an increasingly variety of cancer types. As a result of its mechanism of action, immunerelated side-effects may occur that are important to be distinguished from tumor progression - emphasizing the need for timely detection. In this study, we used deep learning applied to routine clinical CTs for diagnosis of intrapulmonary sarcoid-like granulomatous lesions subsequent to antiCTLA-4 monotherapy.

Methods. A deep learning network developed for lung cancer screening was used and fine-tuned on a cohort of 4579 lung nodules of 138 melanoma patients, of which 1679 lung nodules of 69 patients were used for independent testing(6 diagnostic outcomes).

Results. The network reached 0.68AUC $(p<0.001)$ for histologicallyproven sarcoid-like granulomatous lesions. Its performance could be improved for pulmonary metastases compared to the original screening network $(0.76$ versus $0.61 \mathrm{AUC}, p<0.001)$. These results suggest the presence of treatment-induced morphological changes, not present in the original treatment-naïve dataset. We found significant differences in the network's ability to distinguish between sarcoid-like and post-infection granulomas (0.71AUC, $p<0.001)$, suggesting reliance on inflammation-associated features. The diagnostic score prognostic for 1-year OS (0.70AUC, $p<0.002)$.

Conclusion. Artificial intelligence can improve the diagnosis of sarcoid-like granulomatous lesions. If validated, these findings could enhance the current diagnostic and treatment workup for patients receiving immunotherapy. 


\subsection{Introduction}

Since the approval of the first checkpoint inhibitors in 2011 for stage-IV melanoma patients [Man11; Hod+10; US ; Eurc], cancer immunotherapy rapidly grew to include a larger variety of cancer types. This culminated in 2017 with the first ever approval of a cancer treatment in any solid tumor with high microsatellite instability or mismatch repair deficiency [Mar+19].

Due to the nature of immunotherapeutic agents, inflammations and autoimmune-like disorders are among the most common side-effects, termed immune-related adverse effects. These include immune-related toxicities of the skin, endocrinopathies, hepatotoxicity, and pneumonitis [FPP16]. While some of them pose little risk to the patient (e.g. skin rash) [Gol+16], others could lead to more serious, life-threatening conditions. Pneumonitis, for example, may represent a life-threatening situation [Fra+18], often affecting treatment continuation or (in more critical situations) trigger the administration of corticosteroids and immunosuppressants [LG16; SDL16], with the danger of developing irreversible interstitial lung disease that might limit patient outcome. Timely detection of these adverse effects is therefore essential for the management of patients undergoing immunotherapy [Dim+18].

While an effort has been made to adapt imaging follow-up schemes [Sey+17b], treatment-specific diagnosis of immune-related adverse effects is still limited due to the lack of familiarity of radiologists with this novel treatment modality [Nis+15], and the limitations of routine clinical imaging unable to clearly differentiate immunecompartments (i.e. the cellular composition of the microenvironment) and tissue immune-infiltrates. Due to the unique nature of the therapy and the rapidly increasing number of patients receiving checkpoint inhibitors, the need for novel diagnostic tools to fit treatment specific needs is clear.

We examined lung "sarcoid-like" granulomatous disease as a side 
effect of CTLA-4 checkpoint inhibitors 11, which currently lacks accurate, non-invasive, treatment-specific diagnostic tools. Studies have raised the awareness of increasing incidence of sarcoid-like lesions in malignancies with an incidence rate of 4.3\% [Ask+99; Bon+15; CK07; PGW95; Rei06; Egg+19]. These lesions arise from the checkpoint inhibitor mediated activation of the immune system, and present as nodular inflammations. Granulomatous lesions are not easily distinguishable from metastatic lesions on routine radiological scans, often being mistaken for progression of malignancy [Sid+17]. The reason for this is because they represent with similar imaging morphology on computed tomography (CT) as well as may show activity on positron emission tomography - computed tomography (PET-CT) resulting in a lack of diagnostic specificity of radiological appearance [Egg+19]. In addition, different nodule types have different treatment options. While guidelines have been established for the management of the incidental finding of solitary pulmonary nodule in patients without pre-existing conditions [NO19; Gra+16], the management of lung nodules still remains challenging in these patients [Nai+18] as well as in cancer patients [Gre+17]. Because of this, further imaging followup examinations during cancer immunotherapy are often needed to observe the development of a suspected possible granulomatous lesion, and even then, biopsy often remains the only option for accurate differential diagnosis [Egg+19; Ohs+17].

As standard radiological diagnostic tools do not provide accurate and non-invasive solutions, current evidence in the literature suggests that superior results could be achieved through the usage of artificial intelligence (AI) $[\mathrm{Bi}+19 \mathrm{~b}]$ - more specifically, deep learning. Unlike standard radiological reporting based on qualitative evaluation of visible features, AI methods applied on routine clinical imaging allow for the quantitative evaluation of recurrent imaging patterns - anatomical structures and morphologies, possibly invisible to the human eye that can be linked to the presence of a medical condition. AI effectively allows radiological images to be used as a source of quantita- 
tive, minable data for diagnosis and prognostication. This study aims to evaluate the performance of deep learning methods in the differential diagnosis of (biopsy proven) lung sarcoid-like granulomatous disease of advanced melanoma patients undergoing CTLA-4 checkpoint inhibitors.

\subsection{Materials and methods}

\subsubsection{Study cohort}

For this study, we retrospectively included consecutive patients with stage IIIA-IV melanoma treated with anti-CTLA4 monotherapy (3 or $10 \mathrm{mg} / \mathrm{kg}$ ) every 3 weeks (until disease progression, discontinuation due to toxicity or patient withdrawal) within the Department of Dermatology of the University Hospital of Zurich (USZ; Zurich, Switzerland) from 2012 - 2018. Of those $n=72$ patients were treated within clinical trials (clinicaltrials.gov NTC00636168, NTC01844505, NTC02388906). The remaining number of patients were treated with anti-CTLA4 as standard of clinical care. These patients were included according the ethical approval for the monocentric biodatabank (including imaging assessment and patient outcome) in melanoma patients treated with targeted and/or immunotherapy (EK 647). All patients underwent standardized imaging-based tumor response assessment with contrast-enhanced computed tomography (CT) with a follow up (FU) interval of 8-10 weeks during the treatment. Only patients with diagnosed intrapulmonary nodules and with a pre-existing CT examination at least 1 year prior to the time point of melanoma diagnosis were included. Patient history and clinical data were collected from electronic medical records. Patients with a known history of immunodeficiency and/or autoimmune disease were excluded. Data was collected according to the approval of the local ethics commission (EK 647 and KEK-ZH 2014-0193) and following the guidelines of the Helsinki Declaration on Human Rights with signed patient consent. Data analysis was carried out at the Netherlands 
Cancer Institute (NKI; Amsterdam, The Netherlands) according to the approval of the local institutional review board (IRBd19-083).

\subsubsection{Imaging Acquisition}

CT images were acquired in a supine position in full inspiration. CT scans were performed with 3 different types of scanners: a 128 slice multidetector CT scanner (Somatom Flash, Siemens, Erlangen, Germany), a 128 slice multidetector CT scanner (Somatom Edge Plus, Siemens, Erlangen, Germany), and 64 slice multidetector CT scanner (Somatom AS, Siemens, Erlangen, Germany). The following parameters were used: tube voltage $120 \mathrm{kV}$, automated attenuation-based tube current modulation with a reference tube current-time product of $320 \mathrm{mAs} /$ rotation; pitch 3.2 ; gantry rotation time $0.25 \mathrm{~s}$, collimation $128 \times 0.6 \mathrm{~mm}$ and $64 \times 0.6 \mathrm{~mm}$, respectively. Images were reconstructed with a slice thickness of $2 \mathrm{~mm}$, an increment of $1.6 \mathrm{~mm}$, a soft tissue kernel B36, and slice thickness 1.5, an increment of 1 , a hard tissue kernel B57, respectively. For contrast enhanced CT scans $65 \mathrm{ml}$ iopromide (Ultravist 300, 300 $\mathrm{mg} / \mathrm{ml}$; Bayer Schering Parma, Berlin, Germany) was injected at a flow rate of $2.1 \mathrm{ml} / \mathrm{s}$ followed by $60 \mathrm{ml}$ of saline solution at the same flow rate. Bolus tracking in the descending aorta was performed with a signal-attenuation threshold of $120 \mathrm{HU}$ and a delay of 25 seconds for the arteriovenous contrast phase.

\subsubsection{Imaging assessment, data preparation, and preprocessing}

Read-out of imaging data was performed by two radiologists (LT 4 years of experience, TN 11 years of experience), consulting follow-up scans and clinical data when required. The readout assessment of the radiologists was performed in concordance, meaning unclear lesions were assessed in consensus. Because of this methodologic approach, no inter-observer assessment was evaluated. Each lung nodule was 
marked with a 3-dimensional spherical region of interest (ROI) positioned in the center of the lesion, allowing to analyze the nodule itself and its surrounding anatomical information. Each nodule was assigned with a unique identifier that was kept consistent through its follow-ups for longitudinal tracking. An additional volumetric segmentation of lung nodules was provided only for lesions in the sarcoid-like granulomatous group, due to time constraints.

Recorded anatomic information included: side (left or right lung), lung lobe (upper, lower, middle or lingula), and subpleural versus intrapulmonary space. The intrapulmonary findings were categorized as following (also see Table 3.1) [Nai+18; CSA19]: metastasis, non-specific pulmonary perifissural lymph node, non-specific granuloma (generally post-infection), ground-glass lesion, scar tissue (post-infection or postoperative), focal infectious inflammation, and sarcoid-like granulomatous lesion. Scar tissue was excluded from the dataset, due to scarcity of samples (no samples in the discovery set). Non-specific granuloma were identified as known intrapulmonary lesions already documented on $\mathrm{CT}$ examination performed at least 1 year prior to the diagnosis of melanoma that remained stable during all follow up CTs, these lesions can be partially calcified.

To generate nodule-specific imaging data, each lung lesion was cropped to a cube of $64 \times 64 \times 64$ voxels (isotropic voxel resampling at $0.75 \mathrm{~mm}$ ) according to the location recorded by the readers. Hounsfield units are clipped between -1000 (air density) and 1000 (cortical bone density) and normalized to zero mean and one standard deviation.

\subsubsection{Deep learning}

The dataset was divided into discovery and independent validation set based on patient identifiers. A publicly available 3-dimensional convolutional neural network was used on our dataset ${ }^{1}$. The network was

\footnotetext{
${ }^{1}$ url: github.com/LouisFoucard/DSB17_3d_lung_nodule_classifier
} 
originally trained to classify malignant pulmonary lesions in a publicly available screening imaging dataset. We optimized the original network on our dataset via fine-tuning: the first six convolutional and batch normalization layers were frozen during fine-tuning, and the original multi-layer perceptron was replaced by a global average pooling followed by a softmax classifier. In other words, we employed the original screening network to extract quantitative imaging features, and replaced the original binary classifier (malignant vs benign) with a 6-class classifier, one class per diagnostic outcome. The improvement of the diagnostic performance was evaluated by comparing the performance of the original network on our dataset, to our optimized network for the classification of malignant lesions (as the original network was trained only to distinguish malignant lesions from benign ones with the use of the publicly available LUNA dataset ${ }^{2}$ ). The LUNA dataset is composed of 158 patients with different malignancies, including malignant and benign lesions. Sarcoid-like lesions labeling was not provided. The diagnostic parameters of the LUNA dataset were assessed by a team of expert readers. Biopsies were taken in a number of unclear cases. The LUNA dataset is more heterogeneous, as it contains contrast and non-contrast scans, as well as different voxel sizes and resolution parameters. To reduce overfitting and improve generalization, images were augmented with random rotation of $10^{\circ}$, shift and zoom of $10 \%$, and random axis flipping. Additional regularization was implemented via dropout $(p=0.5)$ on the softmax classifier. To counter class imbalance, focal loss $(\beta, \alpha=2.0,1.0)$ was used during training [Lin+17]. Adam was used for stochastic optimization ( $\mathrm{lr}=$ 0.001) [KB14], with batch size set to 32 samples. A detailed representation of the network is given in Figure 3.1. The trained network output consisted of a group of six probabilities, one per diagnostic outcome: non-specific granulomas, sarcoid-like granulomatous lesions, focal infections, perifissural lymph nodes, ground-glass lesions and metastases. Due to the lack of samples in the training set and/or test set, scars were excluded.

\footnotetext{
${ }^{2}$ url: luna16.grandchallenge.org
} 


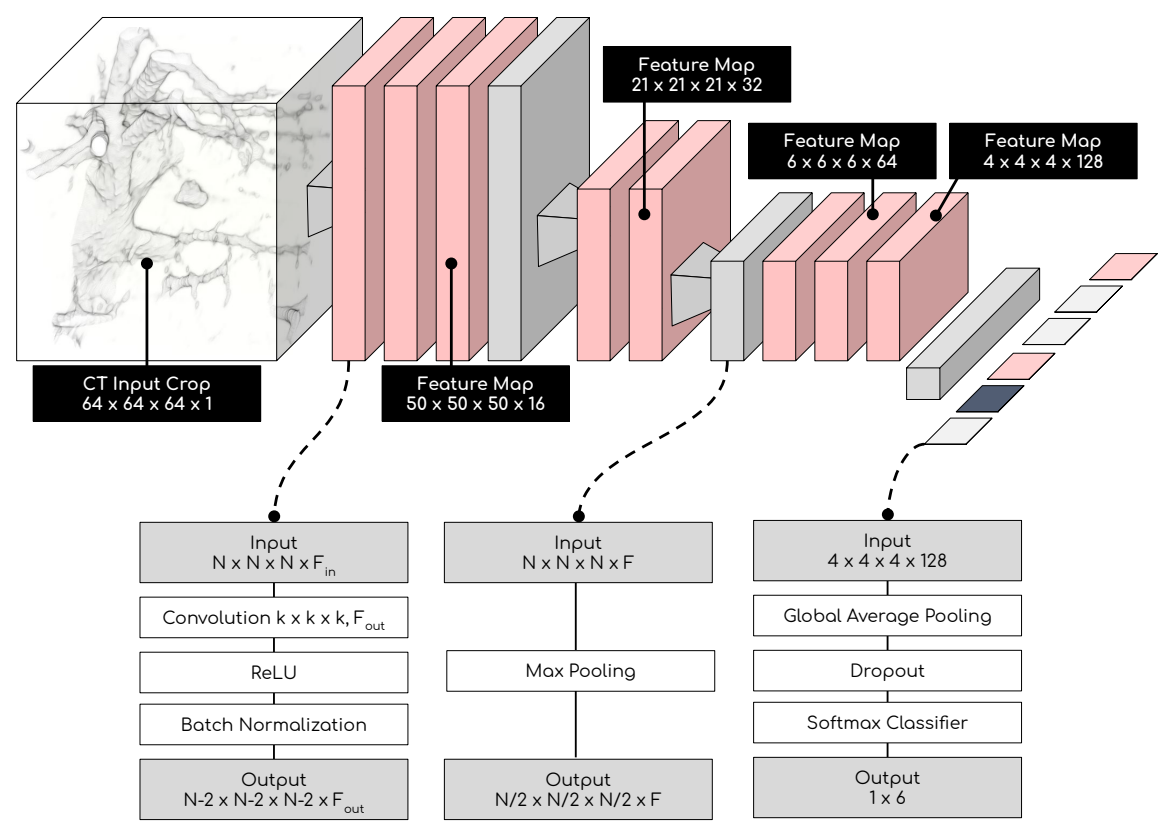

Figure 3.1: Deep learning architecture

\subsubsection{Statistical analysis}

To assess diagnostic performance, each score was analyzed individually against all other diagnostic outcomes (i.e. one-vs-all) using the area under the receiver operating curve (AUC). Confidence intervals were estimated via bootstrapping performed using repeated sampling with replacement (1000 times). Statistical significance was assessed via Mann-Whitney-U test. Further analysis was performed to the score associated with sarcoid-like granulomas lesions against all others diagnostic scores individually (i.e. one-vs-one) using the same statistical metrics. Lesions' malignancy scores generated by the original screening network, and lesions' volumes were used for diagnostic performance comparison. Significant differences in diagnostic performance 
(i.e. between AUCs) were assessed via McNeil test.

Diagnostic scores can be used for both prognostication and stratification. To assess the prognostic performance of our AI-diagnostic signature at baseline (e.g. first available scan), a patient-wide imaging signature was created. In this imaging signature, the highest probability per outcome across all lesions in the scan was recorded. The resulting diagnostic signature described the probability of the patient having at least one lesion with a specific diagnostic outcome (e.g. metastasis, non-specific pulmonary perifissural lymph node, non-specific granuloma, ground-glass lesion, focal infectious inflammation, and sarcoidlike granulomatous lesion). Unsupervised principal component analysis was used to transform the patient-wise signature to a single-value prognostic score. Predictive performance of this score was estimated with Kaplan Meier curves. Statistical significance was assessed via log-rank test. Multivariate Cox regression was used to assess statistical significance against other known diagnostic and clinical parameters used for prognostication, tumor stage, presence of lung metastases and young age $(<65$ years).

\subsubsection{Saliency Maps}

Imaging features used by the network and associated with sarcoid-like granulomatous lesions were analyzed through saliency maps [SVZ13]. Saliency visualization is a technique which highlights sections of the original image that are deemed predictive by the network to estimate the diagnostic score. These highlighted sections are referred to as "salient regions". Saliency maps of sarcoid-like granulomas lesions were generated, along with metastases and non-specific granulomas (similar pathophysiology but different stages of infection) as controls. The qualitative (purely visual) analysis was limited to the top- 8 lesions analyzed by the radiologists per class where the algorithm returned the highest probability of the classification. In other words, these were the eight lesions where the algorithm was most confident in its "diagnosis". 


\subsection{Results}

\subsubsection{Study cohort}

From consecutive $n=293$ patients a total of $n=165$ melanoma patients were included in this study (62 female, median age 63 years, range 55 - 74 years). Twenty-seven patients were excluded due to the lack of visible lung nodules or insufficient image quality. In total, we included $n=138$ patients ( 87 males, 51 females, median age 64 years, melanoma cancer stage IIIA-C N=27, stage IV $N=111$ ), $n=980$ CT scans, and $n=4579$ lung nodules $-n=1059$ baseline nodules longitudinally followed-up with an average of $6 \mathrm{CT}$ scans (IQR=6) every 8 to 12 weeks. Manifestation of granulomatous disease was confirmed through biopsy in $n=8$ patients $(n=74$ CT scans performed in these patients, see Table 3.2). In our study cohort all sarcoid-like lesions CT or PET/CT suggested a malignant cause of these pulmonary nodules, leading into biopsy or thoracic surgery. Also, retrospectively we could not find any imaging pattern that might be specific for sarcoid-like granulomatous disease in our study population. In Figure 3.2c morphologically the overlap between metastases and non-specific granuloma can be seen if compared to the introduced characteristics in Table 3.1. Out of $n=317$ lung nodules of the patients presenting sarcoid-like granulomas disease, $60.57 \%(n=192)$ nodules were sarcoid-like granulomatous lesions. The overall number of nodules per patient in this group does not significantly differ to the negative control (16 versus 17 nodules on average respectively, mann-whitney-u test $p=0.23$ ). The majority of the nodules were either melanoma lung metastases $(n=2772$, $60.54 \%)$, or perifissural intrapulmonary lymph nodes $(n=1150$, $25.11 \%$ ). Non-specific granulomas, sarcoid-like granulomatous lesions, and ground glass lesions were rare, accounting for $7.21 \%$ $(n=330), 4.19 \%(n=192)$ and $1.64 \%(n=75)$ respectively. Because in all patients CT examinations prior to the diagnosis of melanoma were available, all metastases could be identified as new lesions occurring after melanoma diagnosis. All nonspecific lesions like non-specific 
pulmonary perifissural lymph node and non-specific granuloma could be identified in the CT examination at least one year prior to first diagnosis of melanoma. Occurrences of scars, inflammations and lesions shifts (e.g. from perifissural lymph nodes to metastases) were each below $1 \%$. Location-wise, nodules were unevenly distributed (chi-square test, $p<0.001$ ) with lower lobes accounting for half of the nodules $(23.69 \%$ or $n=1085$, and $25.88 \%$ or $n=1185$, for left and right lungs respectively). Nodules in the lingula were the rarest $(4.93 \%$ or $\mathrm{n}=226$, see Table 3.3).

\subsubsection{Diagnostic performance}

The discovery set consisted of $n=69$ patients and $n=2830$ nodules, while the independent test set consisted of the remaining $n=69$ patients ( $n=5$ with sarcoid-like granulomatous disease) and $n=1679$ nodules ( $n=35$ sarcoid-like granulomatous lesions). On the independent test set, the network reached an average performance of 0.69 AUC. Higher performances were reached for ground-glass lesions (0.82 AUC, CI: $0.67-0.9, p=0.002)$, nonspecific granulomas (0.79 AUC, CI: $0.77-0.82, p<0.001)$, and metastases (0.76 AUC, CI: $0.74-0.78, p<0.001)$. Perifissural lymph nodes and sarcoid-like granulomas lesions reached diagnostic performance of 0.69 AUC (CI: $0.67-0.72, p<0.001)$ and 0.68 AUC (CI: $0.61-0.76, p<0.001$ ), respectively. The network performed poorly on focal infectious inflammations (0.40 AUC, CI: $0.27-0.56, p=0.26)$. No significant difference in diagnostic performance has been detected between nodules at first appearance and nodules at follow-ups (McNeil-test, all $p>0.48$ ). Overall, the proposed fine-tuned network significantly outperformed the original one in the classification of metastatic lesions in our test set (0.76 AUC vs 0.61 AUC; McNeil-test, $p<0.001)$ and the volumetric measure within the sarcoid-like granulomatous group (0.76 AUC vs 0.53 AUC; McNeil-test, $p<0.001$ ). Further analysis on diagnostic outcome pairs revealed higher diagnostic performance in distinguishing between sarcoid-like granulomatous lesions versus 
ground-glass lesions (0.82 AUC, CI: $0.59-0.97, p=0.005)$, and sarcoid-like granulomatous lesions versus non-specific granulomas (0.71 AUC, CI: $0.63-0.80, p<0.001$ ). Differential diagnosis of sarcoid-like granulomatous lesions versus perifissural lymph nodes and metastases performed similarly to the general case $(0.69 \mathrm{AUC}$, CI: $0.62-0.76, p<0.001$ and 0.68 AUC, CI: $0.60-0.74, p<0.001$, respectively). The network appears to be unable to distinguish between sarcoid-like granulomatous lesions and focal infection inflammations (0.37 AUC, CI: $0.11-0.71, p=0.22)$. No significant difference in diagnostic performance has been detected between nodules at first appearance and nodules at follow-ups (mcneil-test, all $p>0.57$, see Figure 3.2a). To check that the different scan properties did not interfere with the deep learning model, a sub-analysis within the scans of the granulomatous patients only ( $\mathrm{N}=63$ lesions that were not granulomatous) was performed. The result kept being significant $(p=0.01)$, and similar to the one reported for the entire dataset $(0.64$ AUC, CI: 0.55 - 0.73), which lead us to the conclusion that even when the scan properties are inhomogeneous, the result holds.

\subsubsection{Predictive performance}

Overall median survival of the cohort of patients in the independent test set was 16.6 months. Using the diagnostic score at baseline, we were able to identify two risk groups (i.e. high score and low score, according to score median) with a median survival difference of 12 months (11.3 vs 23.5 months, log-rank-test, $p=0.03)$ and a predictive performance of 0.70 AUC for 1 year overall survival (CI: $0.59-0.81$, $p=0.002)$. In multivariate analysis, the score remained significant for overall survival $(p=0.02)$ against tumor stage ( $>$ stage III, $p=0.08)$, presence of lung metastases $(p=0.43)$ and younger age $(<65$ years, $p=0.87$ ). 


\subsubsection{Saliency maps}

The saliency maps generated for non-specific granulomas, sarcoid-like granulomatous disease and metastases (Figure 3.2c) revealed predictive features in the intralesional periphery, and in the perilesional parenchyma. The intralesional periphery was highlighted in case of sarcoid-like granulomatous-lesions and metastases, whereas imaging features in perilesional parenchyma were deemed predictive by the network in non-specific granulomas. Additionally, in non-specific granulomas a heterogeneous pattern could be depicted compared to the other cases. Salient regions on vasculature (within and around the lesions) could be found across all classes. Anatomical landmarks (e.g. ribs, intercostal muscles, surrounding organs) were not found to be predictive in any of the samples. 

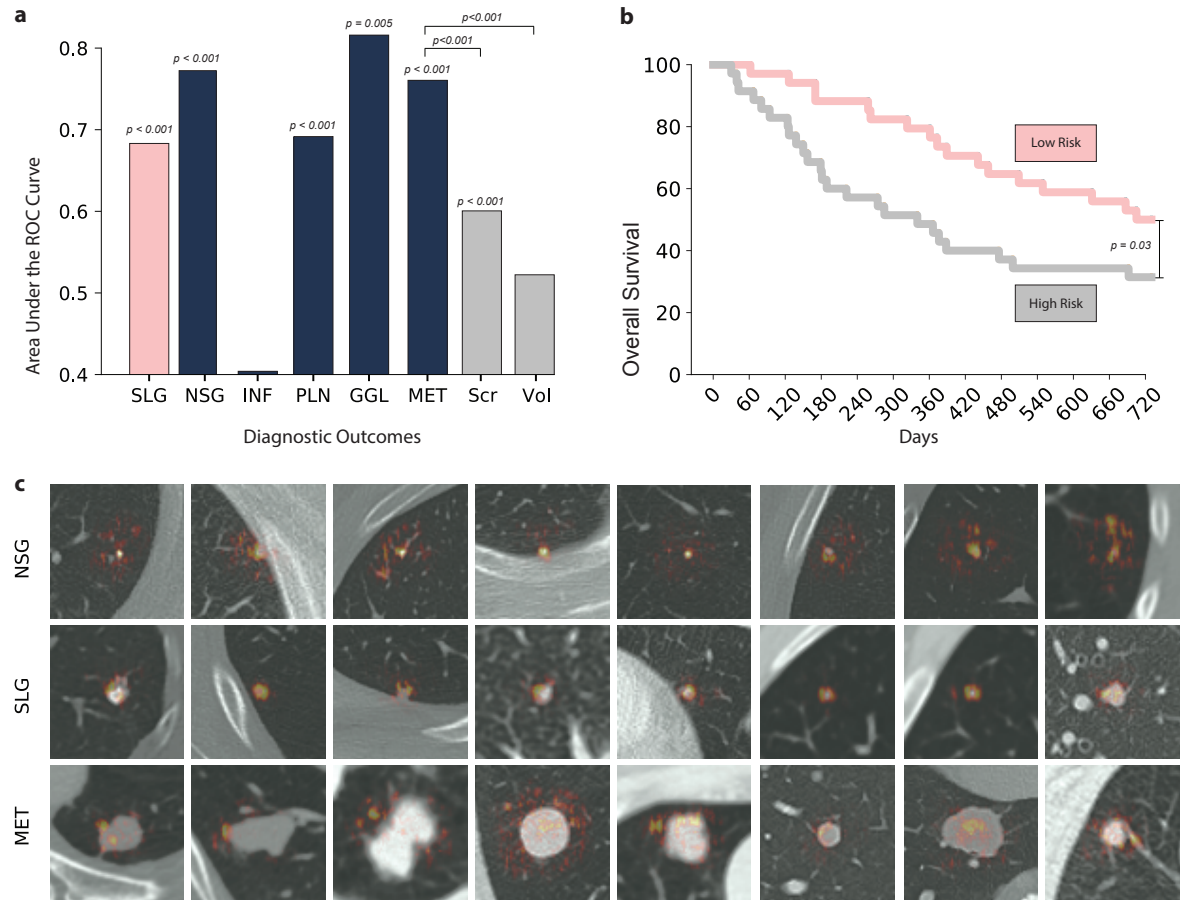

Figure 3.2: (a) Area under the receiver operating characteristic curve of each diagnostic outcomes versus all other diagnostic outcomes. MET metastases (Scr indicates the score of the original, non-fine tuned model trained on screening imaging data, Vol indicates the diagnostic performance of volume), PLN perifissural lymph nodes, NSG non-specific granuloma, SLG Sarcoidlike granulomatous lesions, GGL ground glass lesions, SCA scars, INF infections, SH shifts. (b) Kaplan Meier curves of high and low risk groups, stratified according to the diagnostic score at baseline (c) Saliency maps of sarcoidlike granulomatous lesions and respective controls (metastases and non-specific granulomas). 


\subsection{Discussion}

Cancer immunotherapy checkpoint inhibitors changed the treatment landscape of various cancer types including melanoma. Due to the nature of immunotherapeutic agents, immune-related side effects are common. As these adverse effects could lead to life-threatening conditions, timely detection is essential. While efforts have been made to adapt imaging follow-up schemes, treatment-specific diagnosis is still limited partially due to the novelty of this therapy. Our aim was to evaluate the diagnostic performance of artificial intelligence (deep learning) and medical imaging in the diagnosis of sarcoid-like granulomatous lesions, which arose as an immune-related adverse effect to anti-CTLA4 checkpoint inhibitors in melanoma patients.

Our results show good performance of our AI model for differentiation of sarcoid-like granulomatous lesions against metastases, perifissural lymph nodes, non-specific granulomas, and ground-glass lesions with highly significant AUC-values. Despite the large data imbalance with very few sarcoid-like granulomatous lesions available for training, the careful design of the AI model and training procedure allowed to balance the diagnostic performance of each lesion outcome around 0.69 AUC. No significant difference has been observed in diagnostic performance at nodule baseline compared to their follow-ups, suggesting that the AI model was independent from treatment time-points.

Overall, our network significantly outperformed nodule volume, and visual features in distinguishing cancer lesions from benign lesions. While this difference might have been a direct result of unaccounted changes in the pre-processing steps, image acquisition (e.g. not all patients under screening receive contrast medium) or adjustments in the network architecture and training procedure, one should also account for the discrepancy of patient characteristics. The original network was trained on the LUNA dataset, a publicly available lung screening imaging dataset, containing mostly treatment-naive subjects. It has not yet been proven whether cancer lesions of patients undergoing immunotherapy are subject to morphological changes visible in 
CT images. However, it would be reasonable to assume that the activation of the immune system driven by checkpoint inhibitors might cause changes in the visible (and potentially not-readily-visible) morphology of the tumor as a result of the inflammatory process.

Further analysis revealed higher diagnostic performance of the AI model (up to 0.82 AUC) in the distinction between sarcoid-like granulomatous lesions versus immune-related phenomena, such as non-specific granulomas, and ground-glass lesions. Ground-glass lesions are focal, diffuse, and (partially) solid abnormalities [Hen+02]. Their classification is purely based on radiological characteristics, while the histopathological type might include infection and drug toxicity, sarcoidosis, and malignancy [Hen+02; Kim+13; MS05], among others. The ability of the model in distinguishing between non-specific granulomas and sarcoid-like granulomatous lesions is particularly interesting, as these two nodule types share some similar underlying biological mechanisms. Their difference can be accounted for in two ways. First, non-specific granulomas are generally post-infection, while sarcoid-like granulomatous lesions present an ongoing immune-response. Second, non-specific granulomas are more likely to contain necrotic and fibrotic components, generally not associated with sarcoid-like lesions [IRT07]. Saliency maps of non-specific granulomas displayed predictive imaging features in the surrounding parenchyma and vasculature, whereas sarcoid-like granulomatous lesions showed salient regions in the intralesional periphery. One could hypothesize that the saliency map might depict a still active immune response in the intralesional periphery of the sarcoid-like granulomas lesion in comparison to a possible chronic enclosing immune reaction of the lung parenchyma surrounding the nonspecific granuloma. It is not possible to draw hypotheses, with the same level of certainty, that account for the difference between metastases and sarcoid-like lesions. While sarcoid-like lesions and non-specific granuloma share the same biological basis, cancer lesions lie far apart. Saliency maps clearly depict regions in the intralesional periphery, as well as parenchyma, to be predictive. This 
could, however, be due to a number of factors, including potential differences in vascularization, immune- and tumor-infiltration, morphological features associated with the displacement of healthy tissue, and so on. Further validation is also needed to confirm its biological basis.

Prognostic analysis of the diagnostic score at baseline revealed significant correlation with overall survival, which remained significant even when compared to known negative prognostic factors, such as tumor stage, spread, and age. Diagnostic scores at baseline, such as the radiological tumor stage and pathological TNM classification of malignant tumors, have already been linked to overall survival. In this context, a more complete, quantitative descriptor of the patient status at start of treatment could potentially have prognostic

To the best of our knowledge, this is the first study to investigate the performance of artificial intelligence in the differential diagnosis of intrapulmonary sarcoid-like granulomatous disease in cancer patients undergoing immunotherapy. The earliest case-report of granulomatous disease associated with checkpoint inhibitors was published in 2016 by Danlos et al. [Dan+16] and presented a melanoma patient undergoing anti-PD1 checkpoint inhibitors [Dim+18] who developed a sarcoid-like granulomatous lesion in the mediastinal lymph node and skin after complete response. The authors acknowledged the link between the development of sarcoid-like lesions and the cell-mediated immunity induced by the treatment, while warning of possible adverse clinical implication for sarcoid-like lesions misdiagnosed as tumor progression. Similar reports have been published, including reports for patients receiving anti-PD1 + anti-CTLA4 combinations [Suo+16], and in lung cancer patients receiving anti-PD1 antibodies [Bir+17].

Regarding imaging, evidence in the literature is still scarce. A visual analysis on the morphology of granulomatous lesions in a cohort of 18 patients with common variable immune deficiency presented features, like generalized diffuse reticular pattern and lower lobe predom- 
inance in $80 \%$ of non-specific granulomas positive patients [Par+05]. Molecular imaging, in the form of fluoro-D-glucose positron emission tomography (FDG-PET), is the only reported method for non-invasive, quantitative imaging assessment of sarcoid-like granulomatous lesions $[\mathrm{Cap}+16]$. Activated leukocytes, macrophages and activated helper Tcell (CD4+) show increased FDG uptake, highlighting spots of ongoing inflammation. In these cases, FDG-PET has demonstrated high sensitivity (90\%-100\%). However, translating these results in the differential diagnosis of patients undergoing checkpoint inhibitors is not straightforward, as the method relies on the detection of activated inflammatory components which are likely to be present also in cancer lesions. Although these studies present insights into the viability of imaging for the assessment of sarcoid-like granulomatous lesions, none of them was performed with concurrent, immunotherapy-treated metastatic disease, where cancer lesions are likely to also have sizable active immune-compartments. Clinically, the prospect of routine imaging being able to provide a quantitative, non-invasive diagnostic profile of the whole tumor burden that can also be used in prognostication models is appealing. Such tools would enable clinicians to accurately monitor the treatment and steer it accordingly, while simultaneously avoiding additional invasive (and potentially harmful) examinations for the patient.

\subsubsection{Limitations and future outlook}

The lack of accurate non-invasive diagnostic methods can be explained by several factors. These include the rare (and often asymptomatic) nature of the disease, the novelty of the treatment, and the unfamiliarity of radiologists with treatment side-effects, among others. These factors make data collection complicated, leading to small-sized datasets. The diagnostic accuracy reported and the limited amount of diagnostic outcomes considered in our cohort, would not meet the requirements to use AI alone in the clinical workflow. Better performances are to be expected with increased availability of sarcoid-like granulomatous 
lesion images for training and external validation cohorts. However, due to the rarity of the adverse effect, it is more likely to achieve better performance by leveraging more advanced AI methods, currently under research. For example, additional improvements in the diagnostic performance could be achieved by giving the model access to the clinical history of the patient. This however would require more complex, multi-modal approaches to be investigated separately. Because of the limited dataset in our study no clinical data was included to avoid an unwanted confounder in the evaluation of the performance solely based on imaging features. Additionally, further investigations should include biological validation where the imaging features learnt and leveraged by the AI model are linked back to micro-environmental and genetic quantitative features. While in this study we partially address the problem by leveraging the biological similarity between sarcoidlike granulomatous lesions and post-infectious granulomas, any further conclusion would need to be proven by adequate biological markers, possibly not relying on the generalizability assumption of single lesion biopsy. Finally, in this study we investigated sarcoid-like granulomatous lesions as a side-effect of CTLA- 4 checkpoint inhibitors. Further research in the applicability of this method for treatments focusing on the PD1/PD-L1 axis and combination therapies are required.

\subsection{Conclusions}

Aim of this study was to apply novel technologies of artificial intelligence on routine medical imaging for the diagnosis of sarcoid-like granulomatous lesions induced by novel cancer immunotherapeutic agents. We found significant performance in the diagnosis of sarcoid-like granulomatous lesions, while simultaneously significantly improving the performance of the original screening network for the diagnosis of pulmonary metastases. Moreover, the network was able to distinguish between sarcoid-like granulomatous lesions and non-specific post-infection granulomas. Further investigation is needed to explore the links between the 
imaging features and biological phenomena, and improvement of the diagnostic performance to clinical acceptability. 


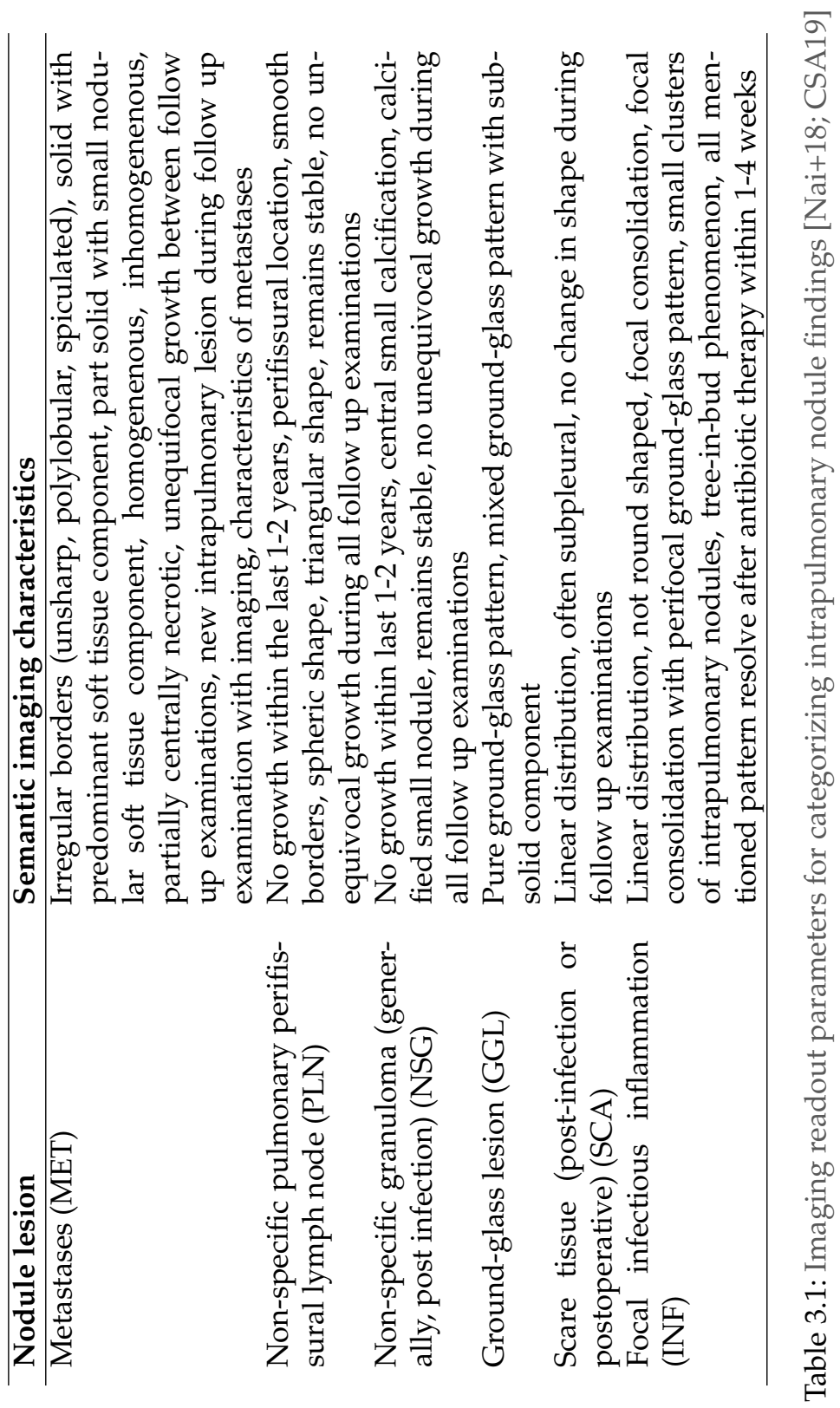




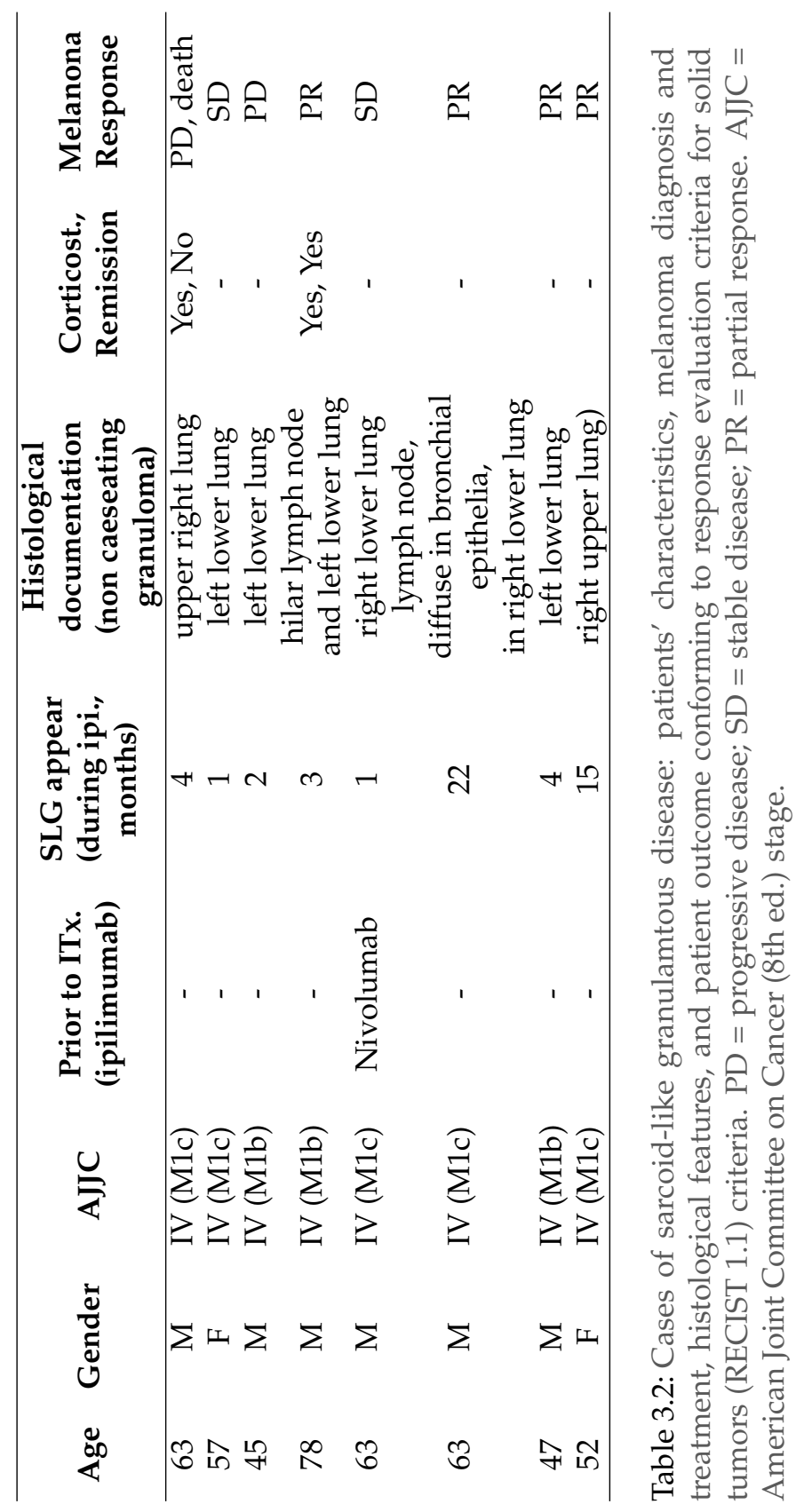




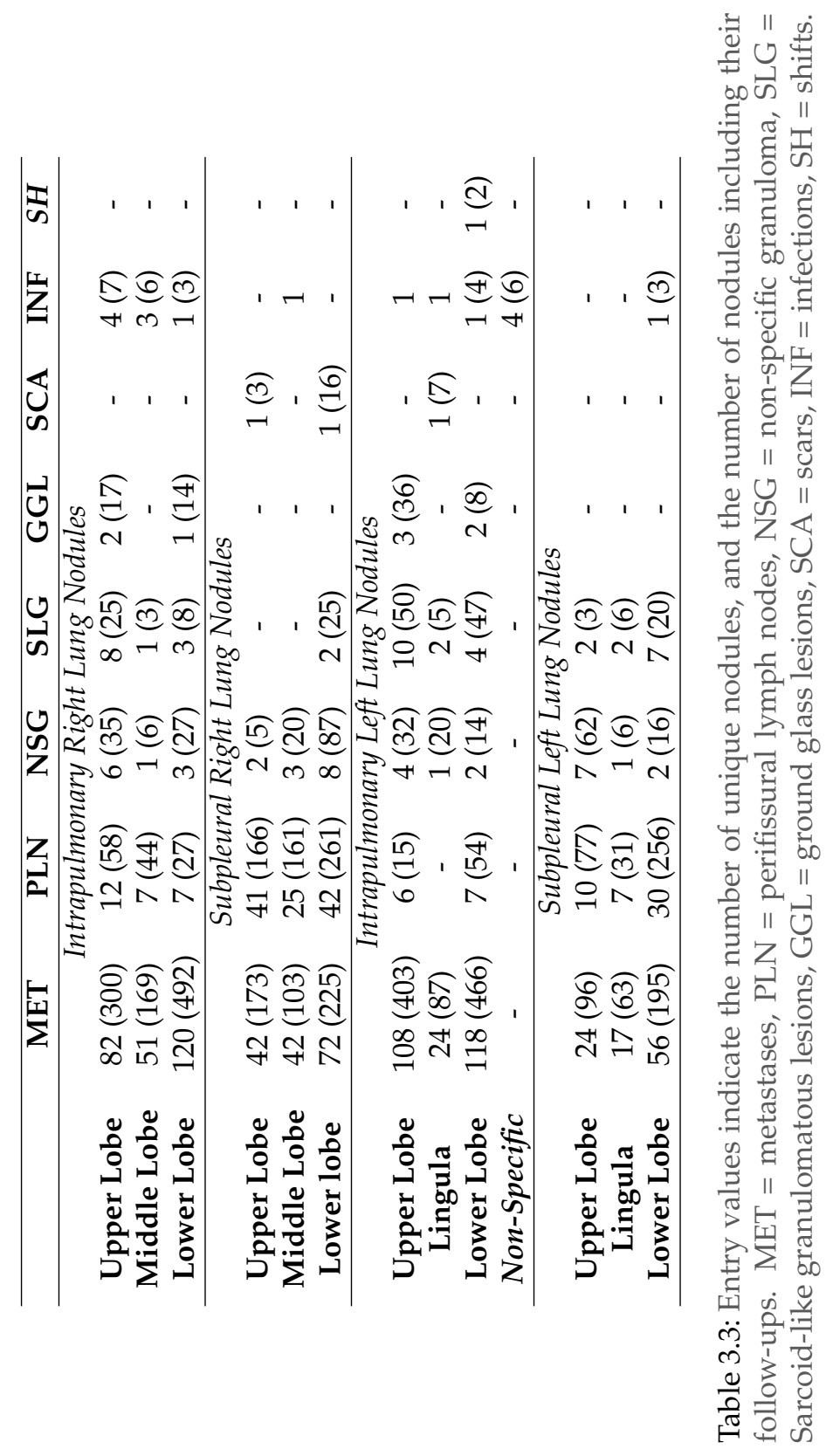




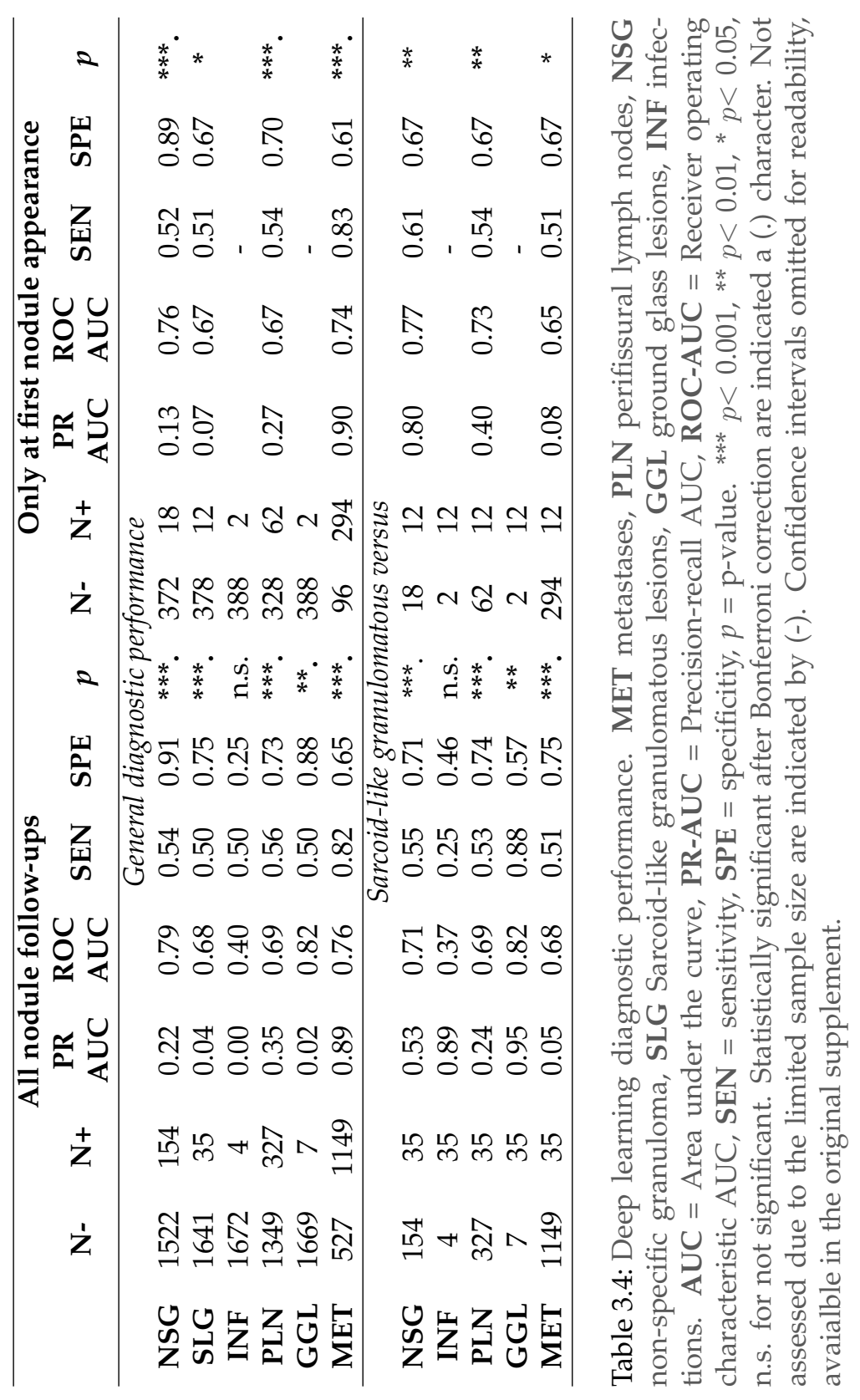





\section{4 \\ Prognostic value of chest imaging monitoring}

Stefano Trebeschi et al. "Prognostic value of deep learning mediated treatment monitoring in lung cancer patients receiving immunotherapy". In: Frontiers in Oncology, accepted for publication (2021). 


\section{Abstract}

Background Checkpoint inhibitors provided sustained clinical benefit to metastatic lung cancer patients. Nonetheless, prognostic markers in metastatic settings are still under research. Imaging offers distinctive advantages, providing whole-body information non-invasively, while routinely available in most clinics. We hypothesized that more prognostic information can be extracted by employing artificial intelligence (AI) for treatment monitoring, superior to 2D tumor growth criteria.

Methods A cohort of 152 stage-IV non-small-cell lung cancer patients (NSCLC)(73 discovery, 79 test, 903 CTs), who received nivolumab were retrospectively collected. We trained a neural network to identify morphological changes on chest CT acquired during patients' follow-ups. A classifier was employed to link imaging features learnt by the network with overall survival.

Results Our results showed significant performance in the independent test set to predict 1-year overall survival from the date of image acquisition, with an average area under the curve (AUC) of 0.69 $(p<0.01)$, up to AUC $0.75(p<0.01)$ in the first $3-5$ months of treatment, and 0.67 AUC $(p=0.01)$ for durable clinical benefit (6-months progression-free survival). We found the AI-derived survival score to be independent of clinical, radiological, PDL1, and histopathological factors. Visual analysis of AI-generated prognostic heatmaps revealed relative prognostic importance of morphological nodal changes in the mediastinum, supraclavicular and hilar regions, lung and bone metastases, as well as pleural effusions, atelectasis and consolidations.

Conclusions Our results demonstrate that deep learning can quantify tumor- and non-tumor related morphological changes important for prognostication on serial imaging. Further investigation should focus on the implementation of this technique beyond thoracic imaging. 


\subsection{Introduction}

Recent advancements in the understanding of the tumor-immune cell interactions [LKA96; Ish+92] have enabled the development of novel drugs for the treatment of advanced-stage lung cancer. Immune checkpoint inhibitors, in particular, have been shown to provide sustained clinical benefit to patients, especially in the metastatic setting [Bor+15; Bra+15; Her+16].

Metastatic markers that can be used for patient selection (i.e. before the start of treatment), as well as for treatment monitoring (i.e. during treatment), are still under research [Ten+18; HCC19b; Ros+19]. In the context of oncological research, most predictive/prognostic markers are derived from tissue samples, routinely-extracted blood [Wan+19], or non-invasive radiological imaging (surrogate imaging markers). Tissue samples derived from biopsies (usually taken from anatomically accessible locations) often fail to account for inter- and intra-lesion heterogeneity, and response assessed during evaluation of tissue samples of only a few lesions does not necessarily mean that all lesions have responded in the same way. Furthermore, serial biopsies during longitudinal follow-up are cumbersome for the patient but also impractical. Regardless of biomarker source, monitoring of response to therapy remains challenging. As such, they are not part of the routine clinical workflow of patients.

Standard clinical imaging provides a non-invasive overview of the entire tumor burden and has the potential to more accurately evaluate the overall response of the patient to the treatment. Yet, imaging evaluation is currently limited to 2-dimensional "subjective" measurements of tumor size changes [Eis+09], time-consuming ROI delineation [Tre+19; Sun+18], and/or to values approximating metabolic activity (i.e. SUV values in PET) [Ten+18]. By limiting the use of imaging for response evaluation to only these approaches, many (potentially prognostic) imaging characteristics are ignored. For example, as the disease evolves in multiple distal sites, traditional imaging assessment methods would not account for 
the microenvironment of each lesion, despite the fact that several potential prognostic factors (e.g. angiogenesis, inflammation, and lymphocytic infiltration) likely depend on that environment [Gar+19]. Since immunotherapy is a systematic treatment modality, changes indicating response are not limited to one location but can occur all over the body. This is particularly relevant in patients treated with anti PD-1 blockade where lymphadenopathy [NHH19; Tir+15], parenchymal inflammations, edema [Ale+19; Joh+16], and compression atelectasis (18), can be observed. Ideally, during image response evaluation these conditions, together with tumor growth, should be monitored and quantified as they might hold valuable prognostic information.

Using Artificial Intelligence (AI), treatment monitoring tools can be built, capable of rapidly assessing gross morphological changes between two (or more) follow-up images of the same patient [Bi+19a], in a fully-automatic manner, completely independent of human input. In this context, image registration can be used as the basis for such a method. At its core, image-to-image registration is the process of establishing a voxel-wise match between two radiological images. By establishing a match, we can measure voxel-level differences between corresponding objects represented in the images quantitatively. While conventional registration techniques are very limited for this application, deep learning-based methods have shown promise in image-toimage registration [HKY20]. There are three main advantages to using deep learning-based image registration as the core technique. The first advantage is that registration networks are trained to match a pair of images, voxel-wise. This creates a network that is explicitly trained to quantify differences between two images. By leveraging its internal features, we can effectively obtain feature vectors that represent these voxel-wise changes. These vectors can be used for classification purposes. The second advantage of using image registration is that it can be trained on large unlabeled datasets (i.e. lacking any kind of manual annotation, such as segmentations or RECIST-like measurements), while not compromising its ability to model voxel-wise details, that are 
likely lost in a classical unsupervised autoencoder approach. The third advantage of using image-to-image registration is that, unlike standard RECIST, such a method could be fully automatic and not require any manual input (e.g. two-dimensional diameter measurements), and not be limited to changes in the tumour size, but it would also account for global morphological changes, whether tumour associated or not, throughout the body. Applying an image-registration-based AI algorithm in oncological follow-up imaging enables us to develop a novel method that can accurately measure gross morphological changes during treatment. Quantitative measurements of these changes can then be used for prognostication.

This study aims to investigate the potential prognostic value of AI-mediated monitoring on CT scans in non-small cell lung cancer (NSCLC) patients receiving anti-PD-1 immune checkpoint blockade. Relying on existing technical research on image-to-image registration, we hypothesize the existence of quantitative imaging features describing a set of gross morphological changes during treatment that hold prognostic value. To test this hypothesis, we developed a deep learning network for thoracic image-to-image registration and studied the prognostic value of features learnt by the network in NSCLC patients being treated with PD-1 blockade.

\subsection{Materials and methods}

\subsubsection{Study cohort}

For this study, we retrospectively included patients with stage IV NSCLC treated with anti-PD1 monotherapy within The Netherlands Cancer Institute - Antoni van Leeuwenhoek Hospital (NKI-AVL; Amsterdam, The Netherlands) between 2014 and 2016. All patients underwent standardized, imaging-based tumor response assessment with contrast-enhanced computed tomography (CT), with follow-up (FU) intervals of 8-12 weeks. We retrieved all available FU scans within the first two years of treatment, together with a baseline 
scan (BL) performed 8 weeks before and up to 1 week after start of treatment. To encode pre-treatment tumor spread, a pre-baseline scan (PBL), defined as the first available scan before $\mathrm{BL}$, was also retrieved when available. The exact dates of each scan were recorded with respect to the start of treatment (in days). Patients with only one scan available throughout the entire treatment regimen, or whose scan would not fully cover the thorax, were excluded from the analysis. The cohort was divided into a discovery and independent test set based on the patient identifier: patients with even ID numbers were assigned to the discovery set, patients with odd ID numbers were assigned to the independent test set. The study was carried out at the NKI-AVL with the approval of the local Institutional Review Board (IRBd19-083). This cohort is a longitudinal expansion of a previously described NSCLC cohort [Tre+19].

\subsubsection{Image acquisition}

The CT scans were performed by either covering the chest or covering the chest and abdomen using multi-slice CT equipment (Toshiba Aquilion CX, Minato, Tokyo, Japan; Siemens Somatom Sensation Open, Erlangen, Germany) with a tube voltage of 120 $\mathrm{kVp}$, slice thickness of $1 \mathrm{~mm}$, and in-plane resolution of $0.75 \times 0.75$ $\mathrm{mm}$. The bolus injection was performed at $3 \mathrm{ml} / \mathrm{s}$ (Omnipaque 300, GE Healthcare, Chicago, Illinois, US) not pre-warmed, with a total amount based on the patient weight $+40 \mathrm{cc}$ (minimum of $90 \mathrm{cc}$ and maximum of $130 \mathrm{cc}$ ) followed by a saline flush of $30 \mathrm{cc}$. The chest CT examinations were performed 40 seconds after contrast injection, whereas the chest and abdomen examinations were performed at 70 seconds.

\subsubsection{Data curation}

Radiological datasets are often heterogeneous. To mitigate differences in radiological image acquisition, all CT scans were cropped between 
the liver and the lower neck region using the method proposed by Zhang et al. [ZWZ17], and linearly resampled to $2 \mathrm{~mm}$ isotropic voxel size. Hounsfield units were clipped between -120 (fat) and 300 (cancellous bone) and rescaled between 0 and 1 . CT scans were further cropped to $192 \times 192 \times 160$ voxels from the center point in order to provide the network with regular image shapes during training.

\subsubsection{Al-mediated quantitative treatment monitoring}

To harness AI for quantitative treatment monitoring, we developed a 3-dimensional convolutional neural network to perform image-toimage registration between subsequent follow-ups of the same patient (architecture shown in Figure 4.1), based on the research of Balakrishnan et al. [Bal+19] and Zaho et al. [Zha+19b]. The network comprised of two subsequent parts: the first performing affine registration aimed to provide alignment of the scans (i.e. to correct for different patient positions), the second section performing deformable registration and aimed to identify morphological changes during the course of the treatment (i.e. longitudinal tracking).

Architecture-wise, the first part of the network consisted of a VGG-like network comprised of a series of five convolutional blocks, and two fully-connected layers, regressing the 12 parameters of the affine transform. The output transform of the network was applied to the moving image, concatenated to the fixed image, and fed into the second part of the network. The second part of the network followed a U-Net architecture [RFB15], and it aimed to quantify non-linear anatomical differences between the input scans. This consisted of an encoding section, comprising 4 convolutional blocks downsampling the images by half the size via striding, a convolutional latent space with stride of 1 , and 4 deconvolutional blocks each upsampling the inputs by double the size via striding. Skip connections were implemented between encoding and decoding layers following the implementation in the original paper. The network was trained to minimize the correlation coefficient loss [Zha $+19 \mathrm{~b}]$. Unlike standard measurements of classical registration 
procedures, this loss is easy to compute in the continuous case. Three penalties were also employed to mitigate for unlikely morphological deformations, each weighted $1 / 10$ in the final loss. Adam optimizer was used during training, with an initial learning rate of $8 \times 10-5$. A curriculum learning scheme was implemented during training, such that the loss would be computed on a smoothed version of the images. The smoothing was implemented via average pooling, starting with a kernel size of 9, and reduced by 3 at epochs 100, 150, and 175. Batch size was set to 2 . To mitigate negative effects resulting from the small batch size, group normalization was employed instead of batch normalization. Figure 4.1 shows a detailed overview of the model loss used. The network was trained on a publicly available dataset of 1010 patients of the lung image database consortium $[\mathrm{McN}+07$; Arm+11; Cla +13 ] with $10 \%$ hold out during training to control for overfitting (i.e. patients whose ID were multipliers of 10 were held out). Our code can be found online ${ }^{1}$.

\subsubsection{Prognostication through quantitative monitoring}

To explore the prognostic value of AI-mediated treatment monitoring, we trained a random forest classifier [B201], with wrapper feature selection, to predict survival based on network imaging features extracted from pairs of subsequent follow-up scans. More specifically, the RFC was trained longitudinally, on pairs of subsequent scans, to predict whether the patient would survive 1 year from the date of the latest of the two scans (see Figure 4.2). The input of the RFC consisted of 96 feature maps from the latent space of the decoder that represented the morphological changes between the prior and the subsequent scan. These are the deepest features found in the middle layer of the second section of the network - the one handling deformable registration. These features come in tensor shape, hence the name feature maps. For classification purposes, it is standard to transform the feature maps of

${ }^{1}$ code: github.com/nki-radiology/PAM.git 
the network to a feature vector, to be fed into a classifier. Global average pooling is the technique commonly used to create a feature vector out of a set of feature maps: each entry of the feature vector is the average value of the corresponding feature map. Alongside the global average pooling, we also included standard deviation, skewness and kurtosis, as we deemed the feature maps too large to be represented just by the mean activation - 1000 values per feature map, compared to 49 of a classical ResNet architecture.

To correct for temporal discrepancies (e.g. differences in time between follow-ups), the amount of days elapsed between the two scans, and the days elapsed since the start of treatment were also fed to the RFC. Furthermore, morphological changes should be order invariant: the differences estimated between image $A$ and $B$ should be the same as the differences between image $\mathrm{B}$ and $\mathrm{A}$. To provide order invariance, we applied element-wise multiplication of the feature maps generated by swapping the input scans. More specifically, we computed the feature maps for the scan pair prior-to-subsequent, and the feature maps for the pair subsequent-to-prior. Then we multiplied them together, element-wise. The multiplication preserved only those changes that were detected in both directions, therefore providing order invariance to our model. The discovery set was used for training, while testing was performed on the independent validation set. Both the registration network and the random forest classifier were trained on the partitioned data, at once, with their respective default parameters - no cross-validation or model selection was performed.

\subsubsection{Prognostic heatmaps}

Occlusion sensitivity was employed to visualize the parts of the image that were deemed prognostic of the outcome [ZF14]. The main idea of the occlusion algorithm is based on the assumption that removing a predictive section / region from the original image will change the algorithm prediction substantially. In contrast, removing a nonpredictive section/region from the original image, the algorithm pre- 
diction will stay unchanged. We occluded a section (or patch) of the input image presented to the RFC. The prognostic value of that patch is then computed as the difference of the RFC survival score produced by the occluded image vs the original unoccluded one. The resulting prognostic map is the result of the algorithm scrolling the ROI through the image, and repeating the procedure. This was filtered with the gross morphological changes map to produce a prognostic map of the gross morphological changes used for visual interpretation. Details of the algorithm reported in Algorithm 1. Visual assessment of the resulting prognostic maps was carried out by an expert reader (T.N.B., board certified radiologist, 2 years experience in thoracic imaging at a tertiary oncologic center), blinded to all clinical parameters, including survival. All scan pairs were assessed with the prognostic maps overlaid on top. The reader was tasked to identify the areas of activation (i.e. hot spots) in the scan pair, and report them categorized as tumor-related areas, secondary comorbidities, and general anatomical areas. Tumor-related areas and secondary comorbidities, which were not highlighted in the prognostic map, were recorded separately.

\subsubsection{Independence from known prognostic factors}

To test the independence of our AI model, we ran a multivariate analysis against known prognostic factors. Age and pathological cancer subtypes were extracted directly from the anonymized patient records. Changes in tumoral burden were computed based on the available manual segmentations of the total tumor - i.e. all visible and segmentable lesions in the body, except for bone and brain. To ensure comparability with $2 \mathrm{D}$ measurements from standard RECIST criteria, volumes where converted to pseudo-diameters via $d=\sqrt[3]{(6 V / \pi)}$, where $\mathrm{V}$ is the total tumoral burden. This computes the diameter of the sphere equi-volumetric to the total tumour burden. Tumor PD-L1 expression scoring was performed according to the instruction manual of the qualitative immunohistochemical assay developed as a complementary diagnostic tool for nivolumab (PD-L1 
IHC 22C3 pharmDx, Dako, Carpinteria, CA). PD-L1 expression levels were determined by observing complete circumferential or partial linear expression (at any intensity) of PD-L1 on the plasma cell membrane of viable tumor-cells. In parallel, the pattern of staining in CD4 stained slides, which also stain CD4+ lymphocytes and macrophages, was evaluated and compared to PD-L1 stained slides in order to avoid false positive assessment due to PD-L1 expressing macrophages in between tumor cells. Assessment of expression levels was performed in sections that included at least 100 tumor cells that could be evaluated.

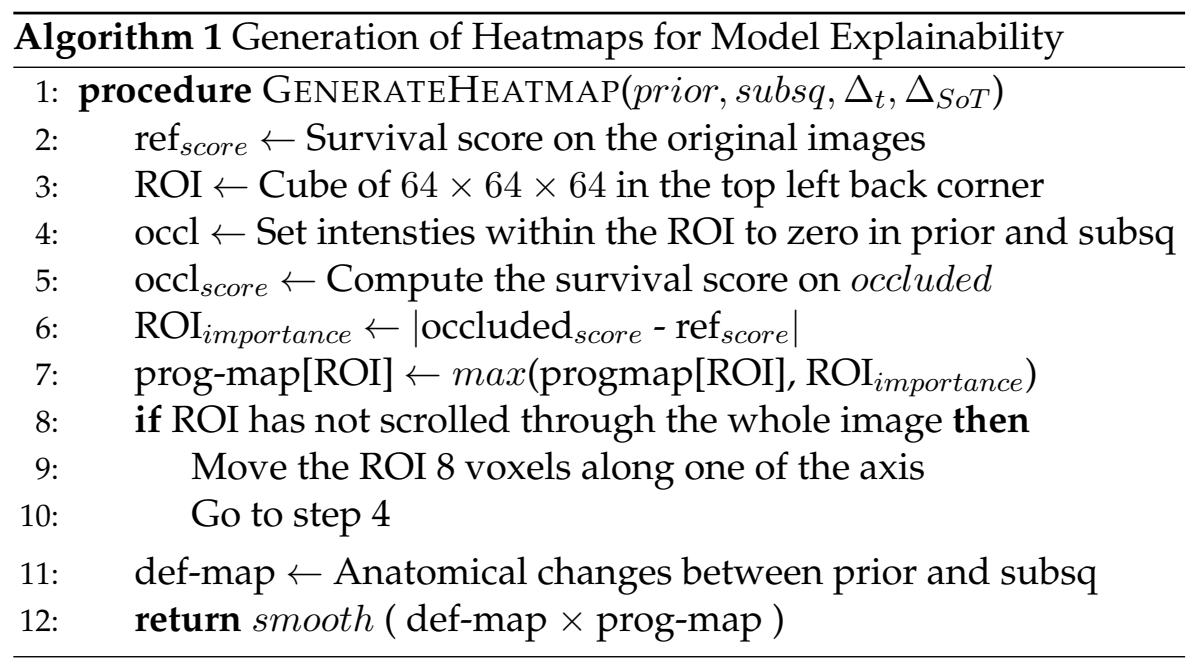

\subsubsection{Statistical analysis}

To assess prognostic performance, the area under the receiver operating curve (ROC-AUC) was used. Confidence intervals were estimated via bootstrapping performed using repeated sampling with replacement (10000 times). Statistical significance was assessed via Mann-Whitney-U test. Kaplan Meier models were employed for survival analysis. Statistical significance of survival metrics 
was assessed via log-rank test. Prognostic (treatment monitoring) performance was quantified in terms of overall survival from the date of the scan. Biomarker performance was quantified in terms of overall survival and durable clinical benefit (complete or partial response, or stable disease, for at least 6 months) from the start of treatment. Cox-Hazards models were used for comparison of known prognostic factors. 

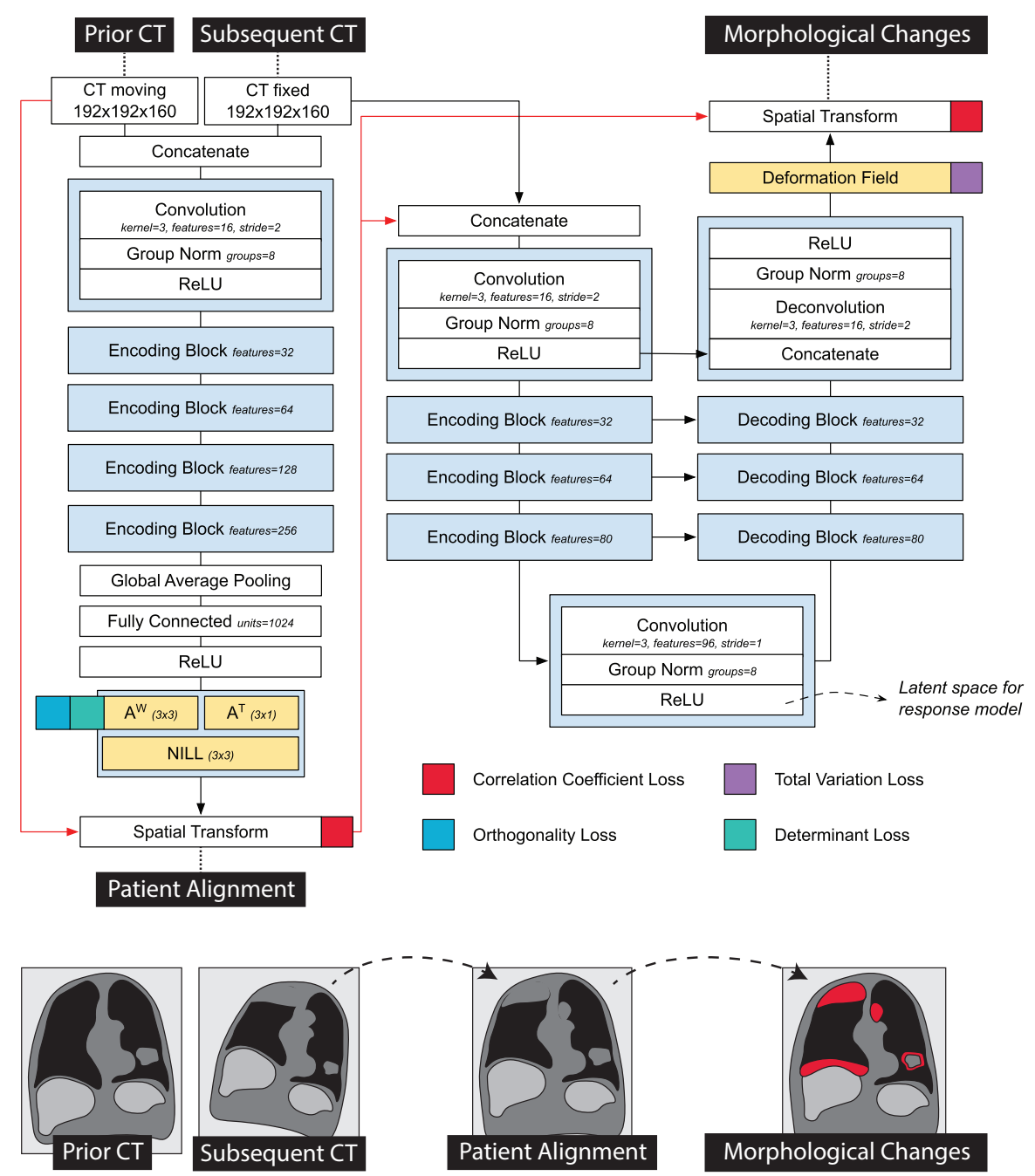

Figure 4.1: Detailed representation of the registration network used in the prognostic AI-monitoring framework. 


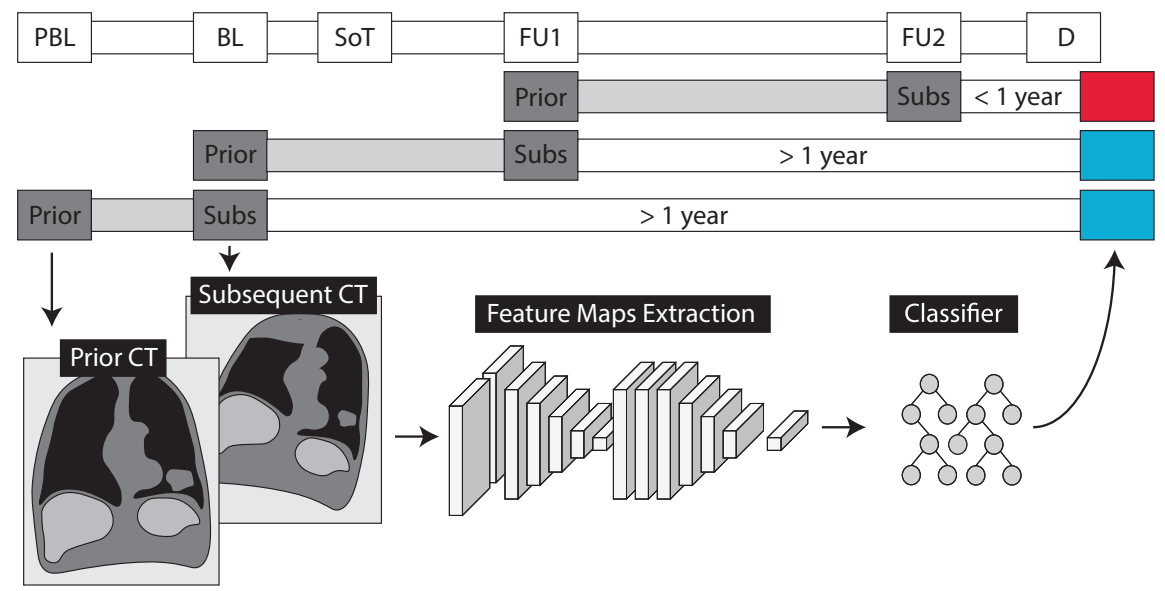

Scan Pairs $\square$ Interval Betwen Scan Pairs $\square$ Survival Time

Survival Classification Outcome

Figure 4.2: Schematic representation of the evaluation of prognostic values through quantitative monitoring. Radiological examinations are shown as pre-baseline (PBL), baseline (BL) and follow-up (FU), with respect to the start of treatment (SoT). Prediction of survival is made based on the time of death (D). For each pair of subsequent scans, we label the earlier one as prior and the subsequent as subsequent (Subs). 


\subsection{Results}

\subsubsection{Study cohort}

A total of $n=152$ patients, $n=903$ CT scans, and $n=611$ scan matched pairs of subsequent CT scans were included in this study (see Figure 4.4). The discovery set consisted of $n=73$ patients (and $n=276$ scan pairs), while the independent validation set had $n=79$ patients (and $\mathrm{n}=335$ scan pairs). Median age of the entire cohort was 64.4 (IQR 57.8 - 68.9), with a higher prevalence of males (57.9\%). Adenocarcinoma was the most common subtype, reported in $61 \%$ of the cohort. No differences in clinical characteristics were encountered between discovery and validation set, except for survival. In comparison to the discovery set, the independent validation set had 180 days longer overall survival, and 101 days longer progression-free survival. Imagingwise, we collected $n=129$ pre-baselines (PBL; 14.3\%), $n=149$ baselines (BL; 16.5\%), $\mathrm{n}=135$ first follow-ups (FU1; 15.0\%), and $\mathrm{n}=103$ second follow-ups (FU2; 11.4\%). Subsequent follow-ups (FU3+) constituted the remaining $42.9 \%$ of the dataset $(n=387)$. Time-wise, $B L$ scans were acquired on average 26 days before the start of treatment (IQR 37 14), while the first FU scan, 68 days after (IQR 46 - 77). Subsequent follow-ups were made on average every 77 days (IQR 55 - 95). Acquisition of non-contrast enhanced PET-CT instead of contrast enhanced $\mathrm{CT}$ was the main reason for lack of imaging during follow-up. Further patient characteristics in Table 4.1 .

\subsubsection{Image registration performance}

We evaluated the performance of the registration algorithm merely to identify the cases where the registration algorithm failed. The evaluation of a registration algorithm is usually performed by evaluating the distance between two known corresponding landmarks in the registered image. This can be done automatically, in a circular fashion. Namely, by selecting $N$ random points in an image, we can transform them to their new coordinates in the target image, and back, using the 
registration functions $T_{A B}$ to represent the transformation from source to target, and $T_{B A}$ as the transformation from target to source. Ideally, these should be the inverse of one another. Practically however there is a registration error propagating from source to target and back. We estimate this error to be proportional to the euclidean distance between $N$ and $T_{B A}\left(T_{A B}(N)\right)$. It is not exactly the registration error, as this depends on two subsequent dependent registration steps. However, as registration is merely the auxiliary task in our model, a full evaluation of the registration procedure - also in terms of architecture and network components - is beyond the scope of this study. The purpose of this analysis is to analyze the worst cases, i.e. the failures of the algorithm.

We ran the evaluation for all scan pairs, with 100 randomly generated points that were transformed from prior to subsequent, and back to prior. The resulting error was $1.67 \mathrm{~cm}$, on average (CI: $0.87-3.18$ ). We selected for visual inspection the three three worst cases, with error 4.54, 3.76 and $3.75 \mathrm{~cm}$, respectively (see Figure in 4.3). This can be considered the closest cases of failure of the algorithm. In each of these cases, we can notice the presence of unlikely deformation, like in the heart or the thoracic wall. Although a penalty was set to deter this behaviour, we would refrain from increasing it, as it might limit the ability of the network to model other deformations. The strength of the algorithm is represented by the classifier able to distinguish informative deformations from non-informative ones. Overall, in other locations of the image, the registration was still successful in matching anatomical structures properly.

\subsubsection{Prognostic performance}

We fed pairs of subsequent follow-up scans to our network trained for (CT chest) image-to-image registration, and trained a random forest classifier (RFC) on its feature maps to investigate the prognostic value of the imaging features learnt by the network. Overall results of the RFC survival score on the independent validation set show an AUC 

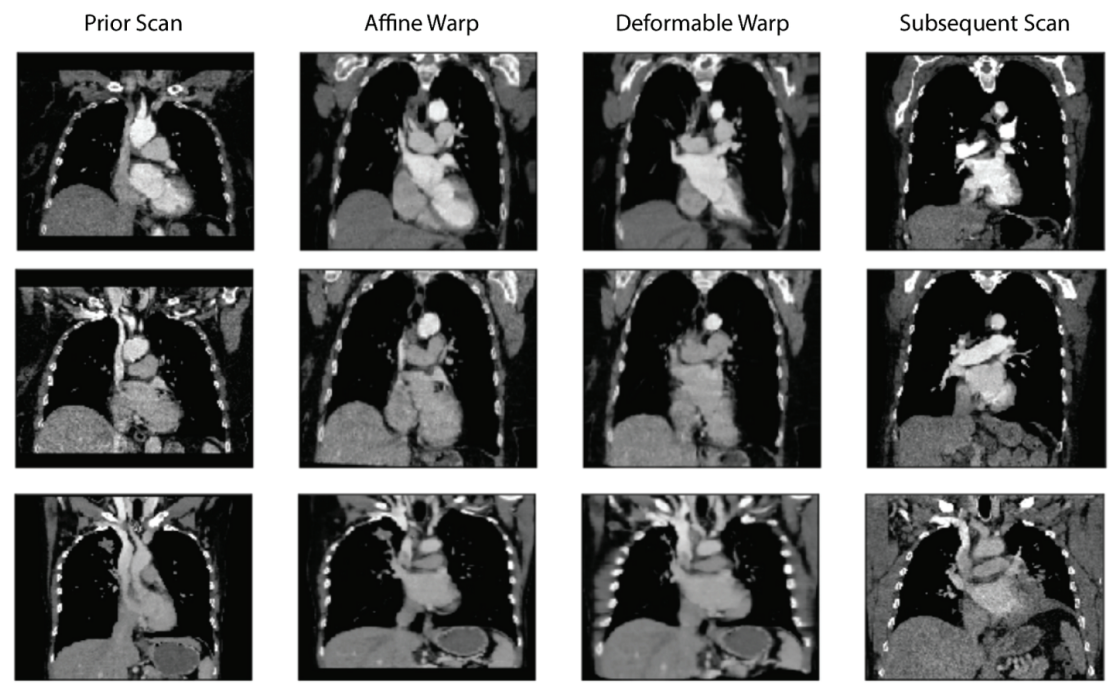

Figure 4.3: The three worst registration cases, and the result at each step of the deep learning registration pipeline.

of 0.68 ( $\mathrm{n}=335$, CI: $0.62-0.74, p<0.001)$ to predict 1 -year overall survival from the date of the later scan of the scan pair (see Figure 4.4b). The highest prognostic value can be found for the scan pair BL-FU1, reaching an AUC of $0.74(\mathrm{n}=61, \mathrm{CI}: 0.61-0.86, p<0.001)$, and for the scan pair FU1-FU2, reaching an AUC of 0.75 ( $n=42, C I$ : $0.58-0.89$, $p=0.002$ ). A decrease in performance is observed during follow-ups, with a 0.71 AUC $(n=42, C I: 0.50-0.89, p=0.02)$ for the pair FU2-FU3. None of these differences however reached statistical significance. Interestingly, RFC survival scores on the pair PBL-BL also showed prognostic value $(0.69$ AUC, $n=51, C I: 0.54-0.83, p=0.01$ ). After the fourth follow-up image, the prognostic performance of the model dropped (0.57 AUC, n=131, CI: $0.47-0.67, p=0.11$ ). This trend becomes evident when looking at the performance with respect to the days between the 
later scan in the scan pair, and the start of the treatment (see Figure 4.4c). In this respect, we divided the exam pairs in five groups, based on the time between the day of the later scan, and the day of start of treatment (i.e. before start of treatment, 0-90 days from start, 90-180 days and $>365$ days), and tested the performance in each group individually. Exam pairs performed before start of treatment showed an AUC of 0.72 ( $n=48, C I: 0.57-0.86, p=0.006)$, between start and 90 days after start of treatment showed an AUC of 0.73 (n=64, CI: $0.59-0.84$, $p<0.001)$, between 90 and 180 days showed an AUC of $0.68(\mathrm{n}=59, \mathrm{CI}$ : $0.51-0.83, p=0.01)$, between 180 and 365 days an AUC of $0.66(n=89$, CI: $0.51-0.79, p=0.01)$. Exam pairs performed in the second year of treatment showed an AUC of 0.63 ( $\mathrm{n}=75, \mathrm{CI}: 0.50-0.75, p=0.04)$. Results summary in Table 4.2.

\subsubsection{Biomarker performance}

To investigate the prognostic value of AI-monitoring as a biomarker we ran a survival analysis on the scan pairs closest to the date of treatment start, i.e. PBL-BL and BL-FU1. High and low risk groups were defined for each scan pair by splitting the RFC survival scores on the median value. The scan pair BL-FU1 offered the highest prognostic performance ( $p=0.02)$, with a median survival difference of 357 days (637 vs 280 days median survival respectively, $p=0.02$, see Figure $4.3 \mathrm{~d}$ ). A similar trend was observed for the PBL-BL pair, with a median survival difference of 239 days ( 467 vs 228 days median survival, respectively, see Figure 4.3e). This, however, did not reach statistical significance $(p=0.16)$. For durable clinical benefit ( 6 months progression-free survival from start of treatment), we ran a classification analysis on the same scan pairs. This yielded a significant performance of 0.67 AUC (CI: $0.52-0.80, p=0.01)$ for the BL-FU1 pair, and a similar trend for the PBL-BL pair (0.61 AUC, CI: $0.44-0.77, p=0.10)$. 


\subsubsection{Combination of multiple time-points}

To investigate the prognostic value of AI-monitoring across multiple time points, we combined the prognostic scores of PBL-BL monitoring, and BL-FU1 monitoring (see Figures 4.3f-g). For this particular analysis, we chose the start of treatment as reference, as differences in follow-up schemas might magnify when combining multiple timepoints. Across the subset of patients analyzed (with PBL, BL and FU1 scans available, $n=43$ ), 53\% survived 1 year after start of treatment $(n=23)$. Patients with high expression of prognostic features during the monitoring of both PBL-BL and BL-FU1 $(n=15)$ showed the highest increase in survival, with an enrichment from the baseline of $27 \%(80 \%$ survived 1 year after start of treatment). On the contrary, patients with low prognostic features on both PBL-BL and BL-FU1 $(n=14)$ showed a diminution from baseline of $24 \%$ (29\% survived 1-year after start of treatment). A point of interest is to be made for patients showing conflicting prognostic scores between PBL-BL and BL-FU1 (positivenegative and negative-positive, $n=7$, respectively). While these groups do not seem to show any deviation from the baseline (50\% survived 1-year after start of treatment), further analysis on OS showed comparable results to the negative-negative group $(p=0.99)$ over a longer time span (2 year, see Figure $4.3 \mathrm{~h}$ ). The positive-positive group, on the other hand, kept showing significantly higher OS compared to both negative-negative $(p=0.01)$ and negative-positive $(p=0.003)$ groups.

\subsubsection{Comparison with known prognostic factors}

To compare the prognostic value of AI-monitoring against other known clinical prognostic factors, we ran a multivariate cox-hazards survival analysis. Specifically, we compared the RFC prognostic scores to age, cancer subtype, volumetric changes in total tumor burden between BL and FU1, and PDL1 expression at baseline. To mitigate collinearity, we reduced PBL-BL / BL-FU1 scores to a single score by principal component analysis. Complete data was available for 22 patients in the independent validation set. Results showed our 
RFC survival score preserved statistical significance $(0.35 \mathrm{HR}, \mathrm{CI}$ : $0.12-0.97, p=0.04)$ against age $(2.69 \mathrm{HR}, \mathrm{CI}: 1.20-6.05, p=0.02)$, volumetric change of total tumor burden $(2.36 \mathrm{HR}, \mathrm{CI}$ : $0.67-8.22$, $\mathrm{p}=0.18),>1 \%$ PDL-1 expression (0.26 HR, CI: $0.03-2.22, p=0.22)$, adenocarcinoma (0.34 HR, CI: $0.03-4.43, p=0.41)$ and squamous subtype (0.14 HR, CI: $0.01-3.01, p=0.21)$.

\subsubsection{Visual inspection of prognostic maps}

The main idea behind predictive maps was to evaluate the predictive value of different regions of the image by removing those regions, one at the time, and estimating the difference in predicted survival. Figure $4.3 \mathrm{i}$ shows an example. The input scans are displayed in the first column. The second column shows the prognostic map generated by the occlusion algorithm (Algorithm 1). The patchy look of the overlay is the result of the cubic ROI, being scrolled around the image. Its intensity values are proportional to the change in predicted survival resulting from occluding that region. The third column is the deformation map, where hotspots correspond to regions of gross morphological changes (i.e. pleural effusion). The fourth column was the visualization presented to the reader. It is the result of the fusion between the prognostic map and the deformation map, and highlights the prognostic changes identified by the algorithm.

At visual inspection, lymph node metastases and lung lesions were common hotspots in the prognostic maps. Nodal metastases were present in $58 \%$ of scan pairs $(n=57)$, and highlighted as prognostic in $81 \%$ of the cases $(n=46)$. The mediastinum contained the most nodal hotspots, being highlighted in $80 \%$ of cases, followed by supraclavicular and hilar nodal metastases, highlighted in $67 \%$ and $57 \%$ of cases respectively. Axillary and pericardial nodal metastases were hotspots in $75 \%$ and $50 \%$ of cases, but found only in $n=4$ and 2 scan pairs respectively. Large lung masses were found in $45 \%$ of scan pairs $(n=39)$, and highlighted as prognostic in $85 \%$ of cases. The same rate was observed for small lung nodules, while being less frequent, found in $30 \%$ of the 
scan pairs $(n=26)$. Bone metastases were found in $20 \%$ of scan pairs $(\mathrm{n}=17)$. Nonetheless, they were deemed prognostic by the algorithm in $82 \%$ of cases. Pleural masses, liver metastases and subcutaneous lesions, while being almost exclusively hotspots in the prognostic maps, accounted together for only 13 scan pairs. Among secondary comorbidities, pleural effusion, consolidations and atelectasis were the most common, accounting for $31 \%, 28 \%$ and $20 \%$ of scans pairs $(n=27,24$, and 17, respectively). Hotspots were found in $94 \%$ cases of atelectasis $(n=16), 93 \%$ cases of pleural effusions $(n=25)$, and $83 \%$ cases of non-specific consolidation $(\mathrm{n}=20)$. Pericardial effusions were hotspots in $75 \%$ of the times, but found only in 8 cases. Only one case of ascites was reported, which the algorithm also highlighted as prognostic. Hotspots in anatomical regions included the spine in $56 \%$ of cases, the thoracic wall in 55\% of cases, and various regions in the upper thorax, including periscapular (51\%), shoulders (49\%), neck (48\%), and supraclavicular (45\%), with the exception of the axilla, highlighted only in $13 \%$ of scan pairs. Normal lung parenchyma was highlighted in $28 \%$ of cases. Remaining hotspots include the great vessels (9\%) and the breast (4\%). Detailed summary reported in Table 4.3. 

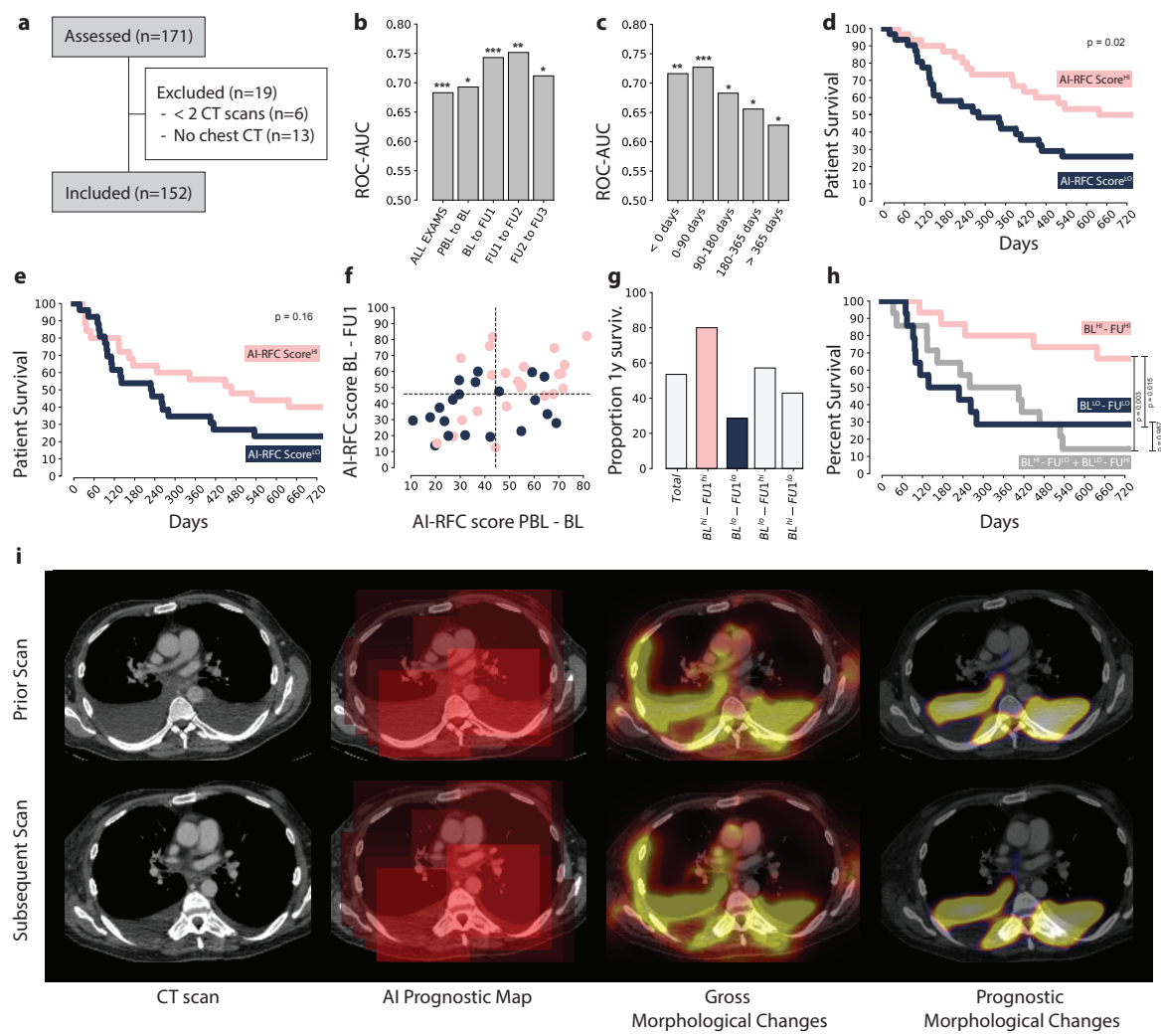

Figure 4.4: (a) CONSORT diagram (b) 1-year survival classification performance on the independent validation set, with respect to the clinical followup routine (highlighted in green the ROC-AUC of the scan pairs used for the 2-years survival analysis) and (c) corrected by time. (d) 2-years Kaplan-Meier curves of the RFC survival score of BL-FU1 and (e) PBL-BL. (f) Combination of the PBL-BL and BL-FU1 RFC survival scores with (g) enrichment of each of the four quadrants (f) and (h) survival of each of the four quadrants. (i) Example of the occlusion sensitivity method used for AI explainability and visualization. 


\subsection{Discussion}

Advanced treatment monitoring through more detailed quantitative descriptors of the overall status of the patient, as visualized on routine imaging scans, could provide valuable prognostic information. Our aim was to investigate the potential prognostic value gained by AIbased treatment monitoring on imaging in NSCLC patients treated by PD-1 checkpoint inhibitors. To test this, we implemented a convolutional neural network for image-to-image registration, and trained it on a large public dataset of chest CT scans. The trained network was then used to longitudinally model gross morphological changes between subsequent scans of NSCLC patients receiving PD1 checkpoint inhibitors. Morphological changes identified by the network were then used to train a classifier to predict 1-year OS from the date of the latest scan.

Our results showed significant performance in the independent test set for the prediction of 1-year OS from the date of image acquisition, with an average AUC of 0.69 , and up to 0.75 AUC for the first 3 to 5 months after start of treatment, and 0.67 AUC for durable clinical benefits, suggesting the presence of (AI-quantified) gross morphological changes encoding prognostic value. These results are comparable to state-of-the-art methods, which currently employs laborious and time-consuming segmentation procedures [Tre+19; Sun+18]. While the field of research has been focusing on single-lesion analysis - leveraging different known factors in cancer growth, including vascularity [Ali+19], oxygenation [Tun+], and metabolic activity [Mu+18] — our approach offers a novel fully automatic procedure which completely eradicates the need of time-consuming segmentations, and simultaneously offers a way to provide a full picture of the patient status as seen on chest imaging. While this does not preclude the usefulness of the single-lesion approach, it proposes a way for future multi-scale solutions that leverage both single lesion imaging biomarkers as well as whole image approaches that provide general quantitative information about the status of the patient receiving treatment. Research ef- 
forts, however, have to be made in order to overcome the bottleneck of manual ROI delineation procedures, either in the form of automatic segmenters [Yan+18], or with implicit AI representations of the total tumor burden.

In addition to the statistical analysis of the performance, we investigated the choices the AI made by means of sensitivity occlusion [ZF14]. This resulted in a set of prognostic heatmaps, highlighting regions of morphological changes that the AI deemed prognostic relevance. Gross morphological changes in nodal and lung lesions held the highest prognostic value, especially nodal lesions in the mediastinum, hilum, and supraclavicular region. Further results suggested additional prognostic value for morphological changes affecting the lungs, either in the form of compression from the thoracic wall (due to pleural effusion or pleural masses), non-specific consolidations, or atelectasis. These results also seemed to extend to other regions, with ascites and pericardial effusions also being highlighted as prognostic, despite their rare occurrence. The AI seemed to pay particular attention to the skeleton, with the spine being the anatomical region most commonly highlighted by the AI in the prognostic maps, and bone metastases deemed prognostic in most cases where those were present. As common imaging follow-up schemas, such as RECIST [Sch+16; Sey+17a], do not account for tumor burden in the bones, our findings suggest that, on the contrary, such phenomena should not be ignored. Further investigations should lead to novel guidelines, which can provide valuable contribution from the imaging beyond diametrical measurements.

Particular attention should also be paid to nodal metastases and nodal growths during treatment. Imaging features of nodal metastases were found already to be correlated with disease progression for NSCLC, melanoma, and head and neck cancer [Tre+19; Yu+19], though no distinction was made between the location of the lymph nodes. However, both our findings and the current literature suggest that this information may be of value. This would be especially interesting in the light of regional (tumor draining) lymph nodes which play a critical role in 
terms of anti-tumor immunity and priming (37), increased expression of cytokines and checkpoint markers [Ho+20a], and changes in the immune compartments resulting in a tumor favorable microenvironment [JPP18]. A major hurdle that remains in the analysis of lymph nodes is represented by the radiological assessment, often in contrast with the pathological one. Most radiomics studies so far focused on the detection of positive nodal metastases rather than their prognostic values [Ho+20b; Sha+20; Tan+19; $\mathrm{Li}+20 ; \mathrm{Zhe}+20 ; \mathrm{Che}+20]$.

The analysis of lung lesions is far more common. Imaging features from lung lesions have been reported to hold prognostic value for patients receiving immunotherapy in several studies [Tre+19; $\mathrm{Mu}+18$; Tan+18; Tun+19; Sun+20b; Pat+19]. Indeed our findings confirm the association between lung lesions and treatment outcome, with about $85 \%$ percent of them being hotspots in the AI-generated prognostic maps, independent of size. Most of the studies published so far focus on the analysis of the tumor region and/or the peritumoral boundary, which may hold valuable information regarding tumor vascularization and inflammatory environment. In this study, the proposed AI model monitors the whole image including both the healthy tissue as well as the tumor(s). As the growth of a cancer lesion does not uniquely depend on the genetic makeup, but rather a complex interaction of microenvironmental features and favorable location for seeding, it would not be surprising to establish a link between a comprehensive modelling tool of cancer growth and its biological features. Even in this case however, further research is needed to establish any link between imaging features and tumor biology.

Following our results, we observed an increase in the prognostic performance of the AI resulting from the combination of multiple time points, namely pre-baseline, baseline and first follow-up. This analysis showed good OS for patients with higher AI-survival scores (AI-RFC ${ }^{\wedge} \mathrm{i}$ ) in both pre-baseline to baseline scan pair, and baseline to first follow-up - and worse OS for the opposite case (AI-RFC ${ }^{\wedge}$ lo). Interestingly, patients with contradicting scores (AI-RFC'hi for pre-baseline to baseline scan pair, and AI-RFC`lo for the baseline 
to follow-up, and vice versa) showed worse survival, similar to the double negative group. These results suggest the existence of a prognostic combination of pretreatment and early-treatment characteristics, both of which should be accounted for during patient stratification. Further insights could be achieved by more advanced AI methods that would account for larger time spans, or even the entirety of patients' treatment history.

The combined score was demonstrated to be an independent prognostic parameter even when corrected for other known prognostic parameters. This is of particular interest when we consider the possible role of such a tool, for example as an additional input to the tumor board during treatment decision making. Further research is required to study its implementation in the clinical settings.

\subsubsection{Limitations and future outlook}

Our study aimed to monitor AI-measured gross morphological changes between imaging follow-up for survival prediction in NSCLC patients receiving PD1 checkpoint antibodies. In this study, we pre-trained a neural network on a large dataset of chest CT scans, and fine-tuned it for survival on our smaller local immunotherapy dataset. Under the current settings, we limited the analysis to chest imaging which, in addition to the chest, frequently included the lower neck and the upper abdomen. While this limitation could hold for lung malignancy, extension to other cancer types would require this technique to be extended to include the whole body - i.e. the abdomen and, when available, the brain. Moreover, due to the limited amount of data, it was not possible to explore more complex machine learning algorithms for the prediction of survival, nor for more precise visualization of the prognostic maps. Expansion of the dataset, both in terms of patients and in terms of time points, would certainly allow for an increase in performance and better explainability of the AI algorithm. Specifically, an extension of the field of view of the algorithm to the whole body, as well as the usage of parameters 
other than imaging, could potentially improve the performance of the algorithm to be usable in the clinics. Further clinical validation of the method is also needed. While this study presented a comparison of this method with response evaluation criteria (e.g. changes in total tumor burden) and biomarkers (e.g. PD-L1 expression), the primary objective for future studies should be a comparison with the clinical standard, namely the RECIST criteria. It remains to be investigated whether this method would be complementary to the current radiological response evaluation (i.e. RECIST). Furthermore, additional investigations are required to link biological features to tumor growth and gross morphological changes. Further analysis should also study the effects of different machine acquisition parameters, and the sensitivity of the method to imaging acquisition parameter variability. Looking into the future, we envision that an AI solution could be set up as a clinical decision support system capable of providing information to the treating physician complementary to traditional clinical and pathological input data.

\subsection{Conclusion}

In this study, we aimed to investigate the potential prognostic value of AI-mediated monitoring in NSCLC patients receiving PD-1 blockade. We hypothesized the existence of quantitative imaging features describing a set of gross morphological changes happening during treatment that hold prognostic information. Our results demonstrate the existence of such factors (as described by the AI on imaging), that are tumor-related, such as nodal, lung and bone lesions, as well as nontumor related, such as pleural effusions, atelectasis and non-specific consolidations. Further investigation should focus on the development of more flexible models that can extend beyond thoracic imaging, as well as on external validations. 


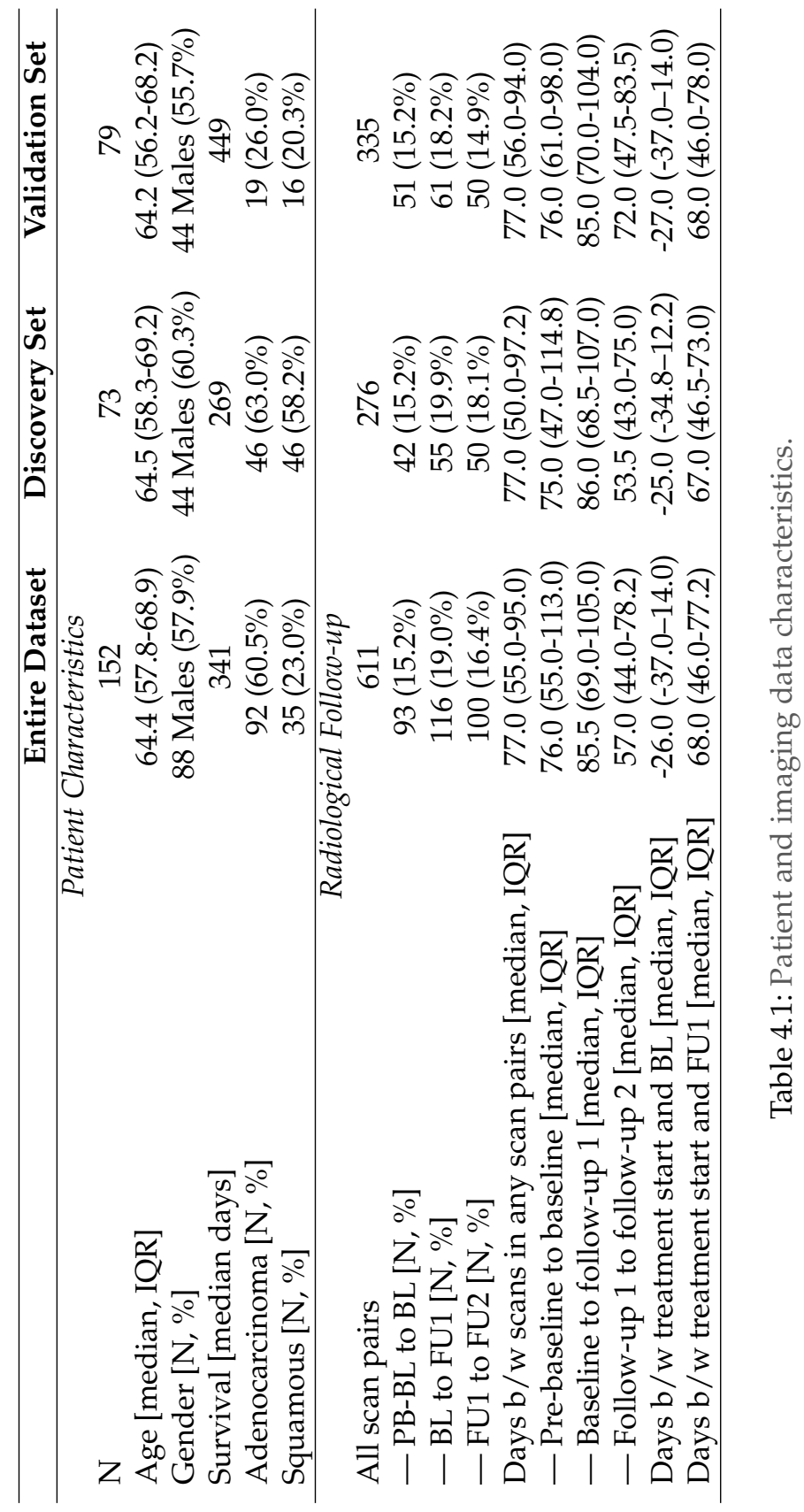




\begin{tabular}{|c|c|c|c|c|}
\hline & N - & $\mathbf{N}+$ & p-value & Area under the ROC curve \\
\hline \multicolumn{5}{|c|}{ With respect to the follow-up sequence } \\
\hline All & 128 & 207 & $<0.001$ & 0.68 (CI: $0.62-0.74)$ \\
\hline $\mathrm{PBL}-\mathrm{BL}$ & 27 & 24 & 0.010 & 0.69 (CI: $0.54-0.83)$ \\
\hline BL - FU1 & 30 & 31 & $<0.001$ & 0.74 (CI: $0.61-0.86)$ \\
\hline FU1 - FU2 & 18 & 32 & 0.002 & 0.75 (CI: $0.58-0.89)$ \\
\hline FU2 - FU3 & 14 & 28 & 0.015 & 0.71 (CI: $0.50-0.89)$ \\
\hline FU3 + & 39 & 92 & 0.112 & 0.57 (CI: $0.47-0.67)$ \\
\hline \multicolumn{5}{|c|}{ With respect to days from start of treatment } \\
\hline$<0$ & 25 & 23 & 0.0057 & 0.72 (CI: $0.56-0.86)$ \\
\hline $0-90$ & 33 & 31 & $<0.001$ & 0.73 (CI: $0.60-0.84)$ \\
\hline $90-180$ & 19 & 40 & 0.013 & 0.68 (CI: $0.51-0.83)$ \\
\hline $180-365$ & 26 & 63 & 0.011 & 0.66 (CI: $0.51-0.79)$ \\
\hline $365+$ & 25 & 50 & 0.037 & 0.63 (CI: $0.50-0.75)$ \\
\hline
\end{tabular}

Table 4.2: Prognostic and predictive performance. 


\begin{tabular}{|c|c|c|c|}
\hline & ALL & PBL - BL & BL - FU1 \\
\hline \multicolumn{4}{|c|}{ Tumor Related } \\
\hline Lymph Nodes & $46 / 57(80.70 \%)$ & $21 / 27(77.78 \%)$ & $25 / 30(83.33 \%)$ \\
\hline — Pericardial & $1 / 2(50.00 \%)$ & $1 / 1(100.00 \%)$ & 0/1 (0.00\%) \\
\hline — Mediastinal & $42 / 53(79.25 \%)$ & $18 / 25(72.00 \%)$ & $24 / 28(85.71 \%)$ \\
\hline - Hilar & $16 / 28(57.14 \%)$ & $7 / 12(58.33 \%)$ & $9 / 16(56.25 \%)$ \\
\hline - Supraclavicular & $16 / 24(66.67 \%)$ & $5 / 10(50.00 \%)$ & $11 / 14(78.57 \%)$ \\
\hline - Axillary & $3 / 4(75.00 \%)$ & $1 / 2(50.00 \%)$ & $2 / 2(100.00 \%)$ \\
\hline Large Lung Nod. & $33 / 39(84.62 \%)$ & $16 / 20(80.00 \%)$ & $17 / 19(89.47 \%)$ \\
\hline Small Lung Nod. & $22 / 26(84.62 \%)$ & $8 / 11(72.73 \%)$ & $14 / 15(93.33 \%)$ \\
\hline Bone Metastases & $14 / 17(82.35 \%)$ & $7 / 7(100.00 \%)$ & $7 / 10(70.00 \%)$ \\
\hline Pleural Masses & $6 / 6(100.00 \%)$ & $3 / 3(100.00 \%)$ & $3 / 3(100.00 \%)$ \\
\hline Liver Metastases & $5 / 6(83.33 \%)$ & $2 / 3(66.67 \%)$ & $3 / 3(100.00 \%)$ \\
\hline Subq. Lesions & $1 / 1(100.00 \%)$ & - & $1 / 1(100.00 \%)$ \\
\hline \multicolumn{4}{|c|}{ Secondary Comorbidities } \\
\hline Pleural Effusion & $25 / 27(92.59 \%)$ & $12 / 12(100.00 \%)$ & $13 / 15(86.67 \%)$ \\
\hline Consolidation & $20 / 24(83.33 \%)$ & $10 / 12(83.33 \%)$ & $10 / 12(83.33 \%)$ \\
\hline - Post-radiation & $3 / 3(100.00 \%)$ & $2 / 2(100.00 \%)$ & $1 / 1(100.00 \%)$ \\
\hline Atelectasis & $16 / 17(94.12 \%)$ & $9 / 9(100.00 \%)$ & $7 / 8(87.50 \%)$ \\
\hline - Post-obstructive & $7 / 8(87.50 \%)$ & $4 / 4(100.00 \%)$ & $3 / 4(75.00 \%)$ \\
\hline Pericardial Effusion & $6 / 8(75.00 \%)$ & $2 / 3(66.67 \%)$ & $4 / 5(80.00 \%)$ \\
\hline Ascites & $1 / 1(100.00 \%)$ & - & $1 / 1(100.00 \%)$ \\
\hline \multicolumn{4}{|c|}{ General Anatomical Areas } \\
\hline Spine & $48 / 86(55.81 \%)$ & $26 / 43(60.47 \%)$ & $22 / 43(51.16 \%)$ \\
\hline Thoracic Wall & $47 / 86(54.65 \%)$ & $25 / 43(58.14 \%)$ & $22 / 43(51.16 \%)$ \\
\hline Periscapular & $44 / 86(51.16 \%)$ & $20 / 43(46.51 \%)$ & $24 / 43(55.81 \%)$ \\
\hline Shoulder & $42 / 86(48.84 \%)$ & $23 / 43(53.49 \%)$ & $19 / 43(44.19 \%)$ \\
\hline Neck & $41 / 86(47.67 \%)$ & $20 / 43(46.51 \%)$ & $21 / 43(48.84 \%)$ \\
\hline Periclavicular & $39 / 86(45.35 \%)$ & $19 / 43(44.19 \%)$ & $20 / 43(46.51 \%)$ \\
\hline Lung Parenchyma & $24 / 86(27.91 \%)$ & $13 / 43(30.23 \%)$ & $11 / 43(25.58 \%)$ \\
\hline Axilla & $11 / 86(12.79 \%)$ & $6 / 43(13.95 \%)$ & $5 / 43(11.63 \%)$ \\
\hline Great Vessels & $8 / 86(9.30 \%)$ & $5 / 43(11.63 \%)$ & $3 / 43(6.98 \%)$ \\
\hline Breast & $3 / 86(3.49 \%)$ & $1 / 43(2.33 \%)$ & $2 / 43(4.65 \%)$ \\
\hline
\end{tabular}

Table 4.3: Results from the visual inspection of the AI-generated prognostic maps. Subq. = subcutaneous, Nod. = Nodule. 


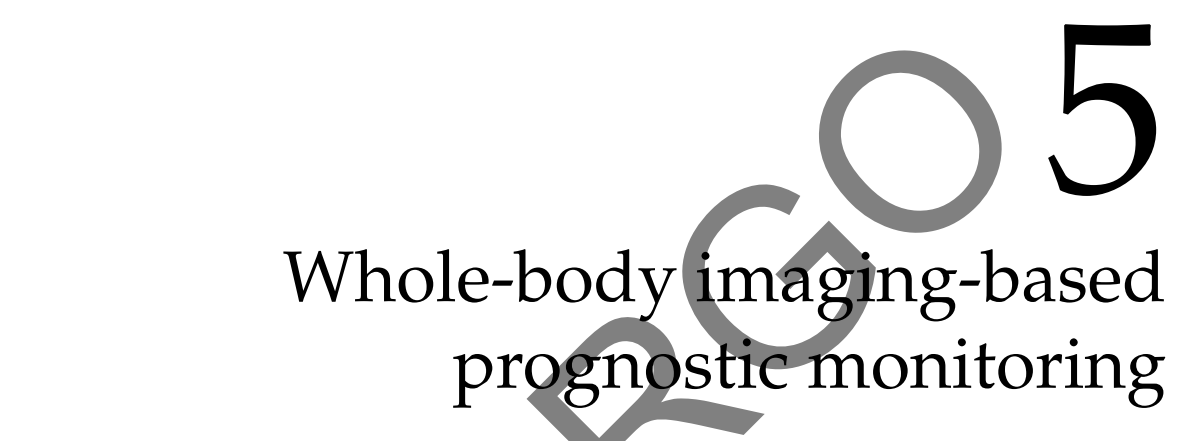

Stefano Trebeschi et al. "Development of a prognostic AI-monitor for metastatic urothelial cancer patients receiving immunotherapy". In: Submitted for publication. (2021). 


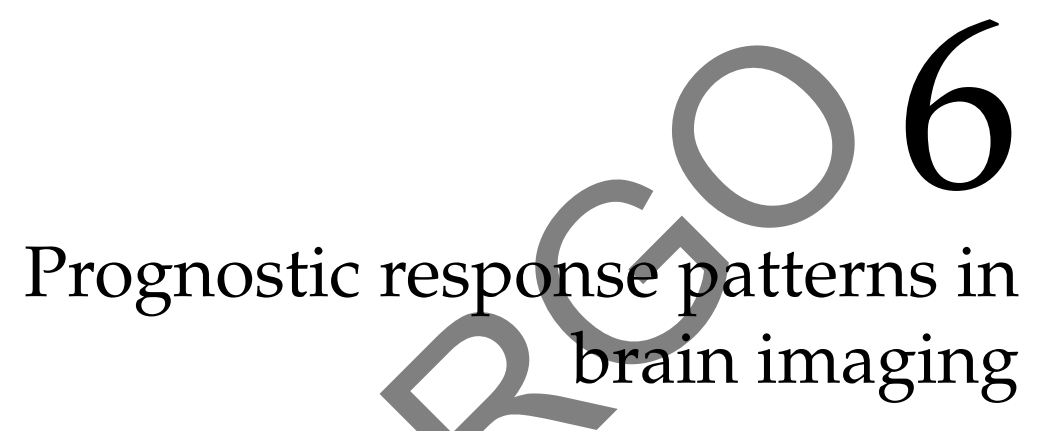

Stefano Trebeschi, Thi Dan Linh Nguyen-Kim et al. "AI-driven identification of prognostic response patterns to immunotherapy of melanoma brain metastases". In: Submitted for publication. (2021). 

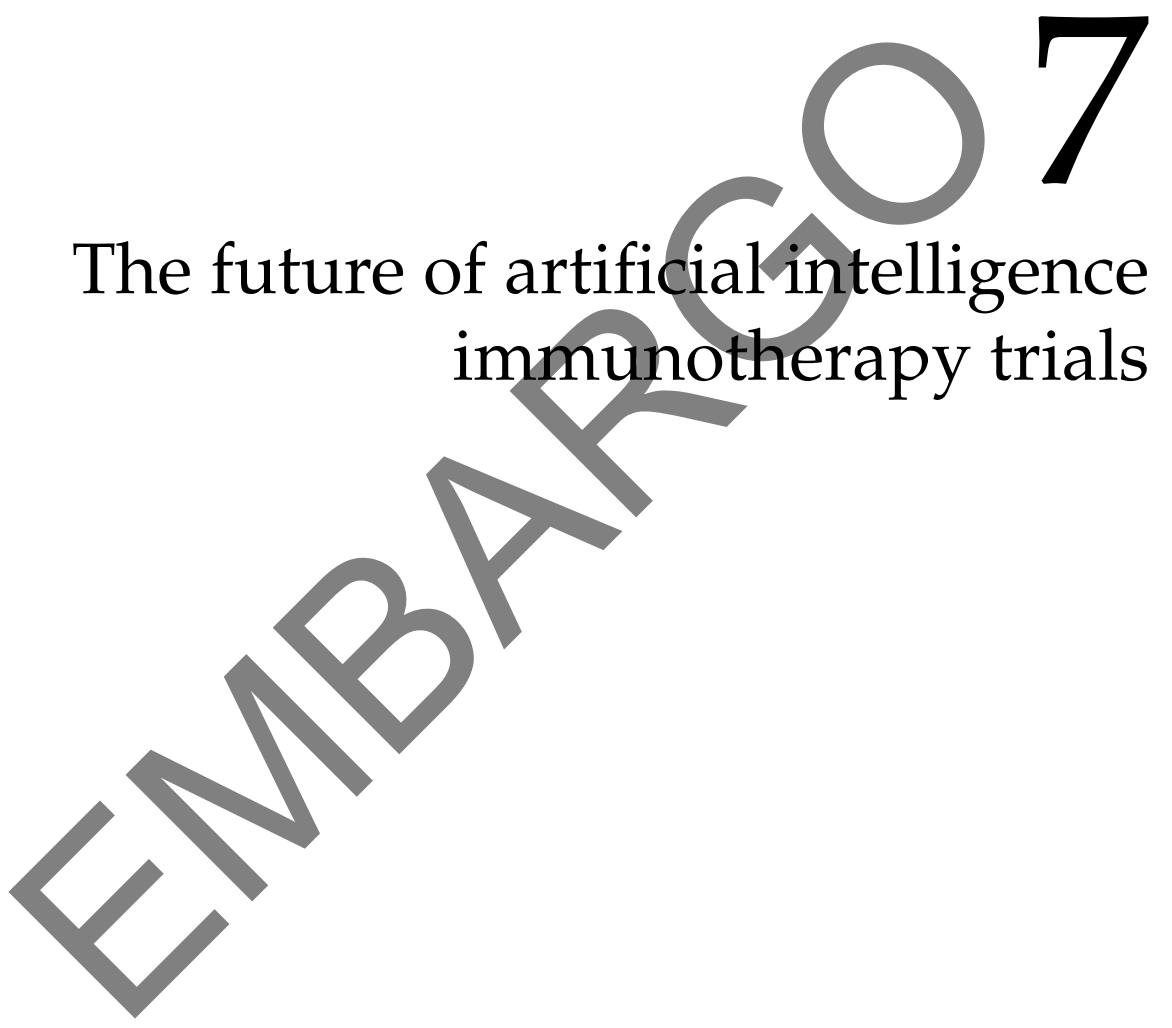

Zuhir Bodalal, Stefano Trebeschi et al. "The future of artificial intelligence applied to immunotherapy trials". In: Neoadjuvant Immunotherapy Treatment of Localized Genitourinary Cancers: Multidisciplinary Management. Book chapter accepted for publication. Springer, 2021. 


\section{8 \\ Towards integrated healthcare}

Zuhir Bodalal, Stefano Trebeschi, and Regina Beets-Tan. "Radiomics: a critical step towards integrated healthcare". In: Insights into imaging 9.6 (2018), pp. 911-914. 


\section{Abstract}

Medical imaging is a vital part of the clinical decision making process, especially in an oncological setting. Radiology has experienced a great wave of change and the advent of quantitative imaging has provided a unique opportunity to analyze patient images objectively. Leveraging radiomics and deep learning, there is increased potential for synergy between physicians and computer networks - via computer aided diagnosis (CAD), computer aided prediction of response (CARP), and computer aided biological profiling (CABP). The ongoing digitalization of other specialties further opens the door for even greater multidisciplinary integration. We envision the development of an integrated system composed of an aggregation of sub-systems interoperating with the aim of achieving an overarching functionality (in this case better CAD, CARP, and CABP). This will require close multidisciplinary cooperation between the clinicians, biomedical scientists, and (bio)engineers as well as an administrative framework where the departments will operate not in isolation but in successful harmony. 


\subsection{Introduction}

Medical imaging has historically played a key role in cancer screening, diagnosis, staging, and therapeutic response monitoring. On a daily basis, treating physicians rely on input from imaging to help formulate patient management plans [Sev+17]. This is especially true within the context of modern oncological guidelines, where patients are stratified into increasingly complex subgroups based on biological, clinical, and radiological parameters.

Historically, qualitative semantic features were used to describe tumour morphology - as observed in the patient image. These descriptions were a reflection of a scoring system based on visual assessment. Semantic features were shown in literature to have correlations with stage, prognosis, and even response prediction [Yip $+17 \mathrm{~b}]$. However, as one could imagine, this method suffered from shortcomings rooted in its dependence on subjective scoring and the limited sensitivity of the human eye.

Modern, ubiquitous imaging modalities, such as CT, MRI, and PET in radiology (and digital images in pathology) are primarily quantitative in nature. This characteristic is harnessed, using computational algorithms, to extract quantitative features and generate mineable data. Rather than relying solely on subjective interpretation of images, these quantitative features can be used to objectively characterize tumour morphology.

In radiomics, medical images are processed to generate quantitative features and this mineable data can then be used for clinical purposes. Radiomic features serve the purpose of describing morphological characteristics (e.g. density distribution, recurrent patterns and textures, shape and outline...) in an objective, quantitative manner. The ambition is to find completely non-invasive radiomic features that could be used as predictive and prognostic biomarkers. 


\subsection{The promise of radiomics}

The advent of radiomics has opened a brand new avenue in cancer research and presents a unique opportunity to data scientists and radiologists alike. Broadly speaking, two prominent potential have emerged for radiomics - tumour characterization and therapeutic response prediction (Figure 8.1).

The search has begun to identify imaging markers that could be used to assess biological parameters (i.e. genetic mutations or surface expression of particular molecules) in the tumour. Normally, such biological assessment of a tumor is achieved by biopsy - a process that is highly invasive, carries potential risk for patient morbidity, and can only elucidate information for lesions in sites easily accessible to surgeons. Radiomics provides the opportunity to non-invasively assess the biological profile (i.e. surface marker expression, genetic mutational status, blood markers etc...) of all the lesions simultaneously and instantaneously. With the increased use of computer models to diagnose conditions and predict response to therapy, this new field where biological parameters can non-invasively be assessed using quantitative features and computer models can be termed Computer Aided Biological Profiling (CABP).

One of the earlier studies to leverage radiomic features in the assessment of genetic mutational status (i.e. radiogenomics) was the work of Segal et al. in human liver cancer where combinations of twentyeight imaging traits were shown to be capable of reconstructing $78 \%$ of the global gene-expression profile (i.e. mRNA levels) of these tumours [Seg+07]. Further research ensued on a number of tumour types - with varying degrees of success.

With rise of deep-learning based image analysis, computer algorithms could be used to extract radiomic features on a large scale which could then be linked to predictive and prognostic biomarkers in cancer (that would otherwise be obtained surgically). 
Unlike more traditional radiomics approaches where feature extraction and data analysis consisted of two separate steps, deep learning fuses these processes together and iteratively optimizes one with respect to the other. In other words, deep learning provides radiomics models with optimal features and optimal data analysis for a specific clinical problem. This advanced form of computer-aided biological profiling (where a neural network can extract features and link them together on a massive scale) can be termed as Deep Learning Mediated Tumour Profiling (DL-TP).

The next application of radiomic features in cancer research was prediction of response to different forms of treatment (i.e. Computer Aided Response Prediction (CARP)). In non-small cell lung cancer (NSCLC), Coroller et al. identified seven features that were predictive for pathological gross residual disease and one feature for pathological complete response [Cor+16]. Further studies later identified other radiomic features that would predict response to conventional treatment (i.e. chemo/radiotherapy) in bladder cancer [Cha+17] and locally advanced rectal cancer [Lov+18]. 


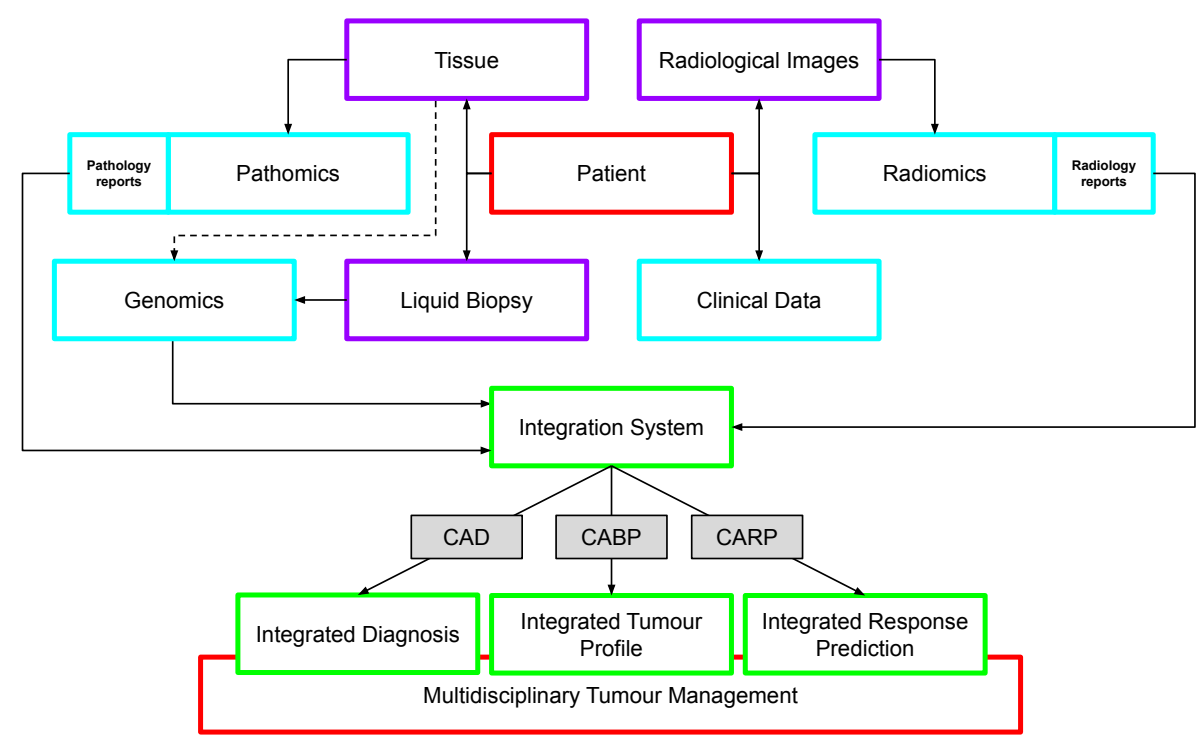

Figure 8.1: A schematic of a future radiomics pipeline highlighting a simplified workflow for CABP and CARP wherein patient images are input into a specialized (series of) AI algorithm(s) and based on the outcome, can be classified. CABP algorithms assess the profile of the tumour (for stratification) while CARP algorithms focus purely on the prediction of response to (and ultimately selection of) therapy. CAD = Computer Aided Diagnosis, CARP = Computer Aided Response Prediction, $\mathbf{C A B P}=$ Computer Aided Biological Profiling.

\subsection{Integrated systems in healthcare}

While the suffix of "-omics" has come to denote the idea of extracting valuable information from datasets, radiomics is only the latest addition to the ever-growing list of new fields of study within the fusion of advanced technology and modern medicine. Images derived from tissue (e.g. general microscopy, immunohistochemistry etc...) have also been subject to quantitative analysis and new information is being generated beyond what would be observed by a pathologist i.e. path- 
omics. Genomics, the branch of molecular biology concerned with the mapping of the human genome, has helped to identify many genetic mutations and pathways that have been used for prognostication or as novel targets for modern therapeutics and has heavily relied on computer models developed by skilled bioinformaticians.

As it currently stands, different medical disciplines have developed different stratification methods, primarily based on their own field (i.e. radiological classifications, pathological and chemical laboratory classifications, clinical checklists used for prognostication etc...) - quite often to the exclusion of other departments. As these traditional scoring systems were often based on subjective interpretations of analogue readouts, combining these disparate outputs is quite challenging. The rise of the quantitative aspects of various medical disciples (i.e. the "-omics") presents a remarkably unique opportunity wherein information from different diagnostic modalities can be objectively integrated.

\subsection{Conclusion}

The long term vision for precision medicine should focus on the development of integration strategies, wherein data derived from the patient themselves could be used to guide the treating physician. Through intricate analyses that integrate clinical data, blood markers, pathomics, radiomics, and genomics, we envision that a patient can be provisionally diagnosed (via computer aided diagnosis), stratified into a molecular subtype of their tumour (via computer aided biological profiling), and have a recommended treatment formulated (via computer aided response prediction). This aggregation of sub-systems cooperating with the aim of achieving an overarching functionality (in this case better CAD, CARP, and CABP) is termed as the integration system (see Figure 8.2). This will require hand-in-hand multidisciplinary collaboration between the biomedical field (i.e. clinicians, geneticists, radiologists, pathologists, clinical chemists), 
and the technical field (i.e. computer scientists, physicists, engineers, statisticians, and mathematicians) as well as an organizational structure wherein the departments will operate not in isolation but in successful integration.
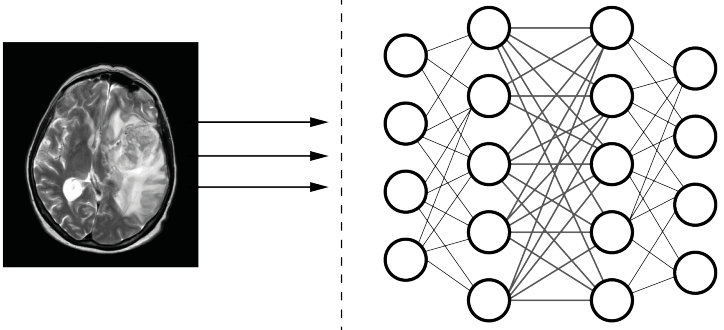

Computer Aided

Biological Profiling

INPUT

PROCESS

OUTPUT

Figure 8.2: A schematic flow chart envisioning the usage of patient-derived data (in light blue) from raw materials (in purple) as a means to improve CAD, CARP, and CABP and ultimately help guide decisions by the multidisciplinary management team. CAD $=$ Computer Aided Diagnosis, $\mathbf{C A R P}=$ Computer Aided Response Prediction, $\mathbf{C A B P}=$ Computer Aided Biological Profiling. 


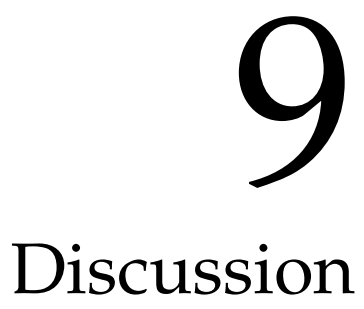


Cancer immunotherapy drugs have been shown to improve outcomes in advanced stage cancer patients [Lar+18; Vok+18; Alb+20; Pai+19]. As these treatments will expand in the future (in terms of the number of monotherapy drugs and their combinations [Bla+18; Mot +19 ; $\mathrm{Hel+19]}$, or by exploiting synergistic effects of non-systemic treatments [PCW19; Twy+15]) the need for personalized medicine, and therefore markers able to select the right treatment for each patient, will increase accordingly [Lüt+20; LN19; MSG17]. Currently, biopsy-derived markers remain the most common type of marker under research [Bai+20; DC19; $\mathrm{McK}+20]$. However, given the invasiveness of the procedure, the risk for sampling error and the fact that it provides information only of few biopsied lesions which does not always reflect the information of the entire tumour load, there is a need for non-invasive biomarkers that could reliable provide us the whole body information on how the patient will respond to treatment [SMH19; Du+19].

We hypothesized that advanced analytical methods of radiomics and artificial intelligence on clinical-routine whole-body imaging data could be used for a non-invasive prediction of treatment response and prognostication of outcome in patients receiving immunotherapy.

To test our hypothesis, we first investigated whether there are lesionwise radiomic computer tomography (CT) features that could be associated with lesion specific biological profiles and tumor growth (chapters 2 and 3). The results would allow us to build an AI model for lesion response prediction in lung cancer and melanoma patients receiving immunotherapy. From these data, we observed that patients with mixed responses (i.e. some lesions growing while others shrinking) were associated with worse survival (chapter 2).

To investigate this further, we set up a different image analysis pipeline, that would enable us to gain insights into different patterns of response, and their relation to survival. We therefore developed an image analytic AI-system, able to quantify whole-body morphological changes in the interval between follow-up scans of the same patient, and investigated whether these changes would be correlated with 
overall survival (chapters 4 and 5). Our results confirmed that the association between response patterns and survival outcomes cannot be explained solely by growth or shrinkage of single-lesions (or a sparse group thereof), nor by a group of tissue-based markers. Instead, our results suggest that specific subsets of lesions, which differ depending on the cancer type, as well as tumour and treatment-induced changes, were equally or better suited to explain overall survival than standard clinical, patho-immunological, and radiological factors (chapters 4, 5 and 6).

In this work, we applied different AI techniques for the study and development of imaging markers and imaging analytic pipelines. Chapter 2 was developed using engineered, handcrafted features, which allowed to focus the analysis to the tumor region only, and prove the existence of predictive imaging features within the tumour - albeit with a significant demand for manual labelling. The region of interest was extended to include the surrounding tissue in chapter 3 . Here, we made use of a more advanced AI method for building the analytic pipeline, namely deep transfer learning. Through visualization techniques, we could observe that the AI algorithm was making use of inner-tumour features, as well as features from the surrounding tissue, highlighting the potential of these advanced techniques to handle more complex data. We took this idea further, by developing an AIalgorithm (chapters 4, 5 and 6 ) able to analyse the whole body - which of course includes tumors and surrounding tissues. We made use of novel deep self-supervised learning to remove the need of manual labelling, and deep learning based image registration to tailor the algorithm to the specific clinical need of response pattern modelling. This led to the development of the prognostic AI-monitor (PAM), which we envision to adapt and extend further into the clinics for imaging and non-imaging data.

The research work in this thesis provided evidence of the existence of morphological patterns in imaging, as processed by AI algorithms, that can function as predictive and prognostic markers in cancer patients receiving immunotherapy. Moreover, our results highlight how 
imaging is the only routinely available tool which allows us to search, localize and connect data patterns across the entire body. Other, more invasive methods, might provide good predictive value as well (despite the fact that this was not observed for both PD-L1 expression, as well as laboratory results), but none of them is able to collect, process and prognosticate based on information that span across the whole body. This is a fundamental aspect in immunotherapy treated patients, as these often present with metastatic disease.

Our work culminates in the development of PAM: an AI-system that allows for comparative analysis between whole-body scans. Conceptually, the closest tool used in the clinics is the Response Evaluation Criteria in Solid Tumour (RECIST) [Sch+16; Eis+09; Sey+17a]. Our findings suggest that aspects that are not included in the current RECISTs can be easily, quickly and fully automatically assessed via AI-radiomics systems, such as PAM. We've proved that in patients receiving immunotherapy, response patterns evaluated from AI methods are equally or better suited to describe survival than approximate tumour growth (i.e. growth corresponds to decreased survival). In this cohort, it is evident that different lesions contribute differently to the prognosis (chapters 4, 5 and 6), suggesting that a different (possibly cancer-specific) selection of target lesions might be needed. It is also evident that non cancer lesions, such as treatmentand cancer-induced complications (chapters 4 and 5) and their diagnostic profiles (chapter 3), should be quantitatively accounted for in response evaluation, as they influence the survival of patients receiving immunotherapy.

A comprehensive overview is also of the current status of immunotherapy imaging marker research is also given in chapter 7. When compared to the work presented in this thesis, we see that single lesions analysis (chapters 2 and 3 ) are the most common in the literature. Response prediction studies have been proposed, with various degrees of novelty, by other studies [Nar+20; $\mathrm{He}+20 ; \mathrm{Maz}+20$; Bas+20; $\mathrm{Mu}+20$; Tun+19; Vai+20; Par+20c], and led to similar results. Most of these studies [Nar+20; Bas+20; $\mathrm{Mu}+20$; Tun+19; Vai+20; 
Par+20c] made use of the original experimental design presented in chapters 2 and 3. Different designs included AI-radiomics models built to predict biological factors relevant to immunotherapy, like tutal mutational burden $[\mathrm{He}+20$; Vee+20], microsatellite instability [Del+20], tumour micro-environment [Maz+20]. Only one study, by Del Re et al. [Del+20] proposed the integration of radiomics to biopsy-derived markers, which led to higher accuracies.

In chapters 4, 5 and 6 we proposed a completely different approach than the one applied in these studies. Conceptually, it could be associated with delta radiomics [Kho+20]. In delta radiomics, the difference between radiomics signature on serial imaging is used for response prediction and prognostication. This however is mostly based on radiomics signatures extracted from time-consuming segmentations, which also limits the analysis to the regions of the tumour, and does not account for any other prognostic or predictive factor that is not a cancer lesion. Instead, our approach is fast, comprehensive, fully automatic, and easily extendable to other imaging types, cancer types and possibly therapies.

\section{The role of $\mathrm{Al}$ in clinical decision making}

None of the aforementioned studies, including ours, address exhaustively how these methods would fit into a possible clinical scenario. The treatment of cancer patients is currently decided in a multidisciplinary meeting of medical specialists, i.e. the tumour board [El +14]. Here, the case of each cancer patient is presented, and discussed. The outcome, a patient-specific treatment and care plan, is the result of diagnostic information, oncologic guidelines [Bes+14; Mic+19; Bel+14] and expert knowledge, carefully knitted together, to achieve an optimal balance of different objectives, namely: prolonged survival, sufficient quality of life, and accomodation of patient's own requests [Sle+90; BTO01; Hir+05; McQ+95; Wee+98; Mar+14]. In this complex interplay of experts and specialties, it might be worth to 
figure out where exactly an AI system like PAM (chapters 4, 5 and 6 ), or the lesion-wise correspondent (chapters 2 and 3), would fit.

The easiest scenario would see the AI being actively interrogated by clinicians in order to estimate the treatment with the highest prognostic likelihood. In this scenario however, it would be unclear to what extent such AI should be trained: should this include only the main treatment, or should this also account for side and palliative ones? The infeasibility of this solution becomes clearer when considering the plethora of treatment combinations that will be available in the near future [JD19; Roc+19; BBB18].

In the short term, a more likely scenario might see the training of main AI systems for the main available drugs (including immunotherapeutic ones). Lesions or side-effects likely to impair the success of the systematic treatment would be treated locally instead, via e.g. radiotherapy or radiofrequency ablation. The usefulness of such an approach is particularly evident in immunotherapy, where patients can remain under treatment up to 1 year, or until there are visible clinical benefits in doing so - namely the disease remains stable and under control [BC18; BKG20; Smi+20]. In this scenario, the AI would therefore accompany the tumour board, quickly analysing baseline and followup data to identify situations of risk to be discussed within the board. Such a scenario would see the clinicians in the board empowered with an "AI-eye", while still retaining control of the treatment planning.

It is important to note that the role of the AI to be played in this scenario does not replace the therapeutic specialities of the tumor board, but rather acts as complementary to them. However, the role of diagnostic specialties in relation to the AI would have to be defined. Our results from the multivariate analyses of chapters IV, V and VI show that currently used clinical methods can be complementary to AI-based methods. While we cannot preclude future development of other AI systems to replace current diagnostic methods and roles, current evidence is insufficient to support the replacement of any clinical role by AI. 


\section{The position of $\mathrm{Al}$ in integrated diagnosis}

The large amount of data generated by current diagnostic departments (chapter 7), as well as the call for integrative diagnostic (chapter 8), requires specialized integrative AI-systems able to process high-dimensional, heterogeneous data, and scale it down, for expert interpretability. In this work, specialists in diagnostic disciplines (i.e. radiologists) were interpreters of AI-machine readings. In a future scenario where an "orchestra" of AIs will be running simultaneously within the department, the diagnostic specialist will have to assume the role of "conductor", collecting the results from different machines, interpreting and filtering them, and sharing them with the tumour board. Only the information relevant to the final objective should be provided to the board.

The objective itself remains the main hurdle. While improvement of survival is an objective, measurable function that can be analyzed mathematically, quality of life and accommodation of patient-specific requests require human comprehension and understanding. To the best of our knowledge, it is still unclear whether, in the near future, there will be an AI-machine able to create treatment plans which, for example, could accommodate even simple requests, such as "regardless of the prognosis, avoid amputation" or "spare bowel control, as much as possible". One could argue that an AI system could be trained to replicate the choices made in past cases. Even assuming that such an AI could be trained, and ignoring all practical issues related to it, such as the lack of necessary data to train such a system (e.g. conversations between physicians and patients), doubts would persist of whether this scenario were the wisest. Current accomplishments in cancer treatment are the results of years of multidisciplinary dialogue between different fields of science, which has the physicians as central coordinating nodes, thanks to their practical experience with patients. Were this node to be replaced by an AI trained to replicate treatment plans from the past (or variation of them), the entire innovation process might collapse, with negative 
long term outcomes far overreaching the money saved from a reduction of personnel. We therefore argue that the optimal, and safest scenario is the one proposed, where the $\mathrm{AI}$ and the physician in the tumour board interact, interrogate and improve each other.

In this work, we aimed to assess the predictive and prognostic value of AI-radiomics on routinely available whole-body imaging in cancer patients receiving immunotherapy. Thanks to the usage of these systems, we gained deeper insights into the relation between tumour morphology and tumour response to immunotherapy, and immunotherapy response patterns to overall survival. This culminated in the development of an AI-system, PAM, tailored for follow-up analysis of cancer patients undergoing immunotherapy, and publicly shared with the community.

We envision the shared model to spark additional development from the clinical, as well as technological side. An extension of the current models, for example, to include more cancer types or stages, and different treatments. This process would be straightforward, and require relatively small fine-tuning procedures. It is to be seen if the same procedures and principles can be applied to different, non-imaging data that are routinely available during follow-up, such as laboratory, genetic and tissue data. Namely, if the same principle of tracking changes, evaluation response and relating it to prognostic outcomes using AIsystems still holds for non-imaging data. Even more, the question remains of how these systems would play in an integrative diagnostic scheme, where different AIs, working with different data types and different objectives are orchestrated together to provide clinically relevant information. We encourage further research in these questions prior to a clinical prospective validation.

In conclusion, this work presents a prospective of how AI-radiomics systems could benefit oncological care, with practical application in immunotherapy: one of the most promising, yet challenging treatment modality currently available. We showed not only how these systems can be used for prediction and prognostications, but also how they 
can be used to gain additional medical and oncological insights, which relevance outreaches the original fields of radiology or computer science. 



\section{Bibliography}

[Aer16] Hugo J W L Aerts. "The Potential of Radiomic-Based Phenotyping in Precision Medicine: A Review". en. In: JAMA Oncol 2.12 (Dec. 2016), pp. 1636-1642.

[AH16] Hugo J W L Aerts and Hugo J W. "The Potential of Radiomic-Based Phenotyping in Precision Medicine". In: JAMA Oncology 2.12 (2016), p. 1636.

[Aer+19] Hugo J W L Aerts et al. Data From NSCLC-Radiomics. 2019.

[Aer+15] Hugo J W L Aerts et al. Data From NSCLC-RadiomicsGenomics. 2015.

[Aer+14] Hugo J W L Aerts et al. "Decoding tumour phenotype by noninvasive imaging using a quantitative radiomics approach". In: Nat. Commun. 5 (2014).

[Afs+19] P Afshar et al. "From Handcrafted to DeepLearning-Based Cancer Radiomics: Challenges and Opportunities". In: IEEE Signal Process. Mag. 36.4 (July 2019), pp. 132-160.

[Ahu19] Abhimanyu S Ahuja. "The impact of artificial intelligence in medicine on the future role of the physician". en. In: PeerJ 7 (Oct. 2019), e7702.

[Alb+20] Laurence Albiges et al. "Nivolumab plus ipilimumab versus sunitinib for first-line treatment of advanced renal cell carcinoma: extended 4-year follow-up of the phase III CheckMate 214 trial". en. In: ESMO Open 5.6 (Nov. 2020).

[Ale+19] Francesco Alessandrino et al. "Frequency and imaging features of abdominal immune-related adverse events in metastatic lung cancer patients treated with PD-1 inhibitor". en. In: Abdom Radiol (NY) 44.5 (May 2019), pp. 1917-1927. 
[Ali+19] Mehdi Alilou et al. Quantitative vessel tortuosity radiomics on baseline non-contrast lung CT predict response to immunotherapy and are prognostic of overall survival. 2019.

[Ana+17] V Anagnostou et al. "Immuno-oncology trial endpoints: capturing clinically meaningful activity". In: (2017).

[Arm+11] Samuel G Armato et al. The Lung Image Database Consortium (LIDC) and Image Database Resource Initiative (IDRI): A Completed Reference Database of Lung Nodules on CT Scans. 2011.

[ABB19] Moran Artzi, Idan Bressler, and Dafna Ben Bashat. “Differentiation between glioblastoma, brain metastasis and subtypes using radiomics analysis". en. In: J. Magn. Reson. Imaging 50.2 (Aug. 2019), pp. 519-528.

[Arv+16] Nils D Arvold et al. "Updates in the management of brain metastases". en. In: Neuro. Oncol. 18.8 (Aug. 2016), pp. 1043-1065.

[Ask+99] Johan Askling et al. "Increased risk for cancer following sarcoidosis". In: American Journal of Respiratory and Critical Care Medicine 160.5 (1999), pp. 1668-1672.

[Aye+17] Mark Ayers et al. "IFN- $\gamma$-related mRNA profile predicts clinical response to PD-1 blockade". In: J. Clin. Invest. 127.8 (Aug. 2017), pp. 2930-2940.

[Bai+20] Rilan Bai et al. "Predictive biomarkers for cancer immunotherapy with immune checkpoint inhibitors". en. In: Biomark Res 8 (Aug. 2020), p. 34.

[Bak+17] Shaimaa Bakr et al. Data for NSCLC Radiogenomics Collection. 2017.

[Bal+19] Guha Balakrishnan et al. "VoxelMorph: A Learning Framework for Deformable Medical Image Registration". en. In: IEEE Trans. Med. Imaging (Feb. 2019). 
[Bal+17] Arjun V Balar et al. "Atezolizumab as first-line treatment in cisplatin-ineligible patients with locally advanced and metastatic urothelial carcinoma: a single-arm, multicentre, phase 2 trial". In: Lancet 389.10064 (Jan. 2017), pp. 6776.

[BTO01] C E Balmer, P Thomas, and R J Osborne. "Who wants second-line, palliative chemotherapy?" en. In: Psychooncology 10.5 (Sept. 2001), pp. 410-418.

[BC18] Shanta Bantia and Nirmal Choradia. "Treatment duration with immune-based therapies in Cancer: an enigma". en. In: J Immunother Cancer 6.1 (Dec. 2018), p. 143.

[Bas+20] Lucas Basler et al. "Radiomics, Tumor Volume, and Blood Biomarkers for Early Prediction of Pseudoprogression in Patients with Metastatic Melanoma Treated with Immune Checkpoint Inhibition". en. In: Clin. Cancer Res. 26.16 (Aug. 2020), pp. 4414-4425.

[Bei+15] Reinhard R Beichel et al. Data From QIN-HEADNECK. 2015.

[Bej+17] Babak Ehteshami Bejnordi et al. "DEEP LEARNINGBASED ASSESSMENT OF TUMOR-ASSOCIATED STROMA FOR DIAGNOSING BREAST CANCER IN HISTOPATHOLOGY IMAGES". en. In: Proc. IEEE Int. Symp. Biomed. Imaging 2017 (Apr. 2017), pp. 929-932.

[Bel+14] J Bellmunt et al. "Bladder cancer: ESMO Practice Guidelines for diagnosis, treatment and follow-up". en. In: Ann. Oncol. 25 Suppl 3 (Sept. 2014), pp. iii40-8.

[Ber+16] Anna S Berghoff et al. "Immune Checkpoint Inhibitors in Brain Metastases: From Biology to Treatment". en. In: Am Soc Clin Oncol Educ Book 35 (2016), e116-22. 
[Bes+14] B Besse et al. "2nd ESMO Consensus Conference on Lung Cancer: non-small-cell lung cancer first-line/second and further lines of treatment in advanced disease". en. In: Ann. Oncol. 25.8 (Aug. 2014), pp. 1475-1484.

[BBB18] Neeraj Bhalla, Rachel Brooker, and Michael Brada. "Combining immunotherapy and radiotherapy in lung cancer". en. In: J. Thorac. Dis. 10.Suppl 13 (May 2018), S1447-S1460.

[Bha+19] Ankush Bhatia et al. "MRI radiomic features are associated with survival in melanoma brain metastases treated with immune checkpoint inhibitors". en. In: Neuro. Oncol. 21.12 (Dec. 2019), pp. 1578-1586.

[Bi+19a] Wenya Linda Bi et al. "Artificial intelligence in cancer imaging: Clinical challenges and applications". en. In: CA Cancer J. Clin. 69.2 (Mar. 2019), pp. 127-157.

[Bi+19b] Wenya Linda Bi et al. "Artificial intelligence in cancer imaging: clinical challenges and applications". In: CA: $a$ cancer journal for clinicians 69.2 (2019), pp. 127-157.

[Bil+19] Mehmet A Bilen et al. "The prognostic and predictive impact of inflammatory biomarkers in patients who have advanced-stage cancer treated with immunotherapy: Inflammatory Biomarkers in Immunotherapy". In: Cancer 125.1 (2019), pp. 127-134.

[BKG20] Salem Billan, Orit Kaidar-Person, and Ziv Gil. "Treatment after progression in the era of immunotherapy". en. In: Lancet Oncol. 21.10 (Oct. 2020), e463-e476.

[Bir+17] Mathew R Birnbaum et al. "Nivolumab-related cutaneous sarcoidosis in a patient with lung adenocarcinoma". In: JAAD Case Reports 3.3 (2017), pp. 208-211.

[Bla+18] Christian U Blank et al. "Neoadjuvant versus adjuvant ipilimumab plus nivolumab in macroscopic stage III melanoma". en. In: Nat. Med. 24.11 (Nov. 2018), pp. $1655-1661$. 
[BJJ15] B Nicolas Bloch, Ashali Jain, and Conrade Carl Jaffe. Data From BREAST-DIAGNOSIS. 2015.

[BTB18] Zuhir Bodalal, Stefano Trebeschi, and Regina Beets-Tan. "Radiomics: a critical step towards integrated healthcare". In: Insights into imaging 9.6 (2018), pp. 911-914.

[Bod+19] Zuhir Bodalal et al. "Radiogenomics: bridging imaging and genomics". In: Abdominal Radiology 44.6 (2019), pp. 1960-1984.

[Bon+15] Martina Bonifazi et al. "Sarcoidosis and cancer risk: systematic review and meta-analysis of observational studies". In: Chest 147.3 (2015), pp. 778-791.

[Bor+15] Hossein Borghaei et al. "Nivolumab versus Docetaxel in Advanced Nonsquamous Non-Small-Cell Lung Cancer". en. In: N. Engl. J. Med. 373.17 (Oct. 2015), pp. 1627-1639.

[Bos+15] Walter R Bosch et al. Data From Head-Neck_Cetuximab. 2015.

[Bra+15] Julie Brahmer et al. "Nivolumab versus Docetaxel in Advanced Squamous-Cell Non-Small-Cell Lung Cancer". en. In: N. Engl. J. Med. 373.2 (July 2015), pp. 123-135.

[B201] L Breiman - Machine learning and 2001. "Random forests". In: Springer (2001).

[Bro+20] Tom B Brown et al. "Language Models are Few-Shot Learners". In: (May 2020).

[CB18] Federico Cabitza and Giuseppe Banfi. "Machine learning in laboratory medicine: waiting for the flood?" In: Clin. Chem. Lab. Med. 56.4 (2018), pp. 516-524.

[Cap+16] Selene Capitanio et al. "PET/CT in nononcological lung diseases: current applications and future perspectives". In: European Respiratory Review 25.141 (2016), pp. 247-258. 
[Car+17] David P Carbone et al. "First-Line Nivolumab in Stage IV or Recurrent Non-Small-Cell Lung Cancer". en. In: N. Engl. J. Med. 376.25 (June 2017), pp. 2415-2426.

[Cha+17] Kenny H Cha et al. "Bladder cancer treatment response assessment in CT using radiomics with deep-learning". In: Scientific reports 7.1 (2017), pp. 1-12.

[Cha+18a] Yu Jin Cha et al. "Prediction of Response to Stereotactic Radiosurgery for Brain Metastases Using Convolutional Neural Networks". en. In: Anticancer Res. 38.9 (Sept. 2018), pp. 5437-5445.

[Cha+18b] Stéphane Champiat et al. "Hyperprogressive disease: recognizing a novel pattern to improve patient management". en. In: Nat. Rev. Clin. Oncol. 15.12 (Dec. 2018), pp. 748-762.

[Che+17] Bojiang Chen et al. "Development and clinical application of radiomics in lung cancer". en. In: Radiat. Oncol. 12.1 (Sept. 2017), p. 154.

[Che+20] Jiaming Chen et al. "Noninvasive CT radiomic model for preoperative prediction of lymph node metastasis in early cervical carcinoma". en. In: Br. J. Radiol. 93.1108 (Apr. 2020), p. 20190558.

[Cla+13] Kenneth Clark et al. “The Cancer Imaging Archive (TCIA): maintaining and operating a public information repository". en. In: J. Digit. Imaging 26.6 (Dec. 2013), pp. 1045-1057.

[Coc+19] Kim Cocks et al. “A Q-TWiST Analysis Comparing Nivolumab and Therapy of Investigator's Choice in Patients with Recurrent/Metastatic Platinum-Refractory Squamous Cell Carcinoma of the Head and Neck". en. In: Pharmacoeconomics 37.8 (Aug. 2019), pp. 1041-1047. 
[Coh+20] I Glenn Cohen et al. "The European artificial intelligence strategy: implications and challenges for digital health". en. In: Lancet Digit Health 2.7 (July 2020), e376-e379.

[Coh+16] J V Cohen et al. Melanoma Brain Metastasis Pseudoprogression after Pembrolizumab Treatment. 2016.

[CK07] Philip R Cohen and Razelle Kurzrock. "Sarcoidosis and malignancy". In: Clinics in dermatology 25.3 (2007), pp. 326-333.

[Cor+16] Thibaud P Coroller et al. "Radiomic phenotype features predict pathological response in non-small cell lung cancer". In: Radiotherapy and oncology 119.3 (2016), pp. 480486.

[CT19] Tricia Cottrell and Janis M Taube. "PD-L1 and Emerging Biomarkers in PD-1/PD-L1 Blockade Therapy". In: (2019), p. 14.

[Cou+18] Nicolas Coudray et al. "Classification and mutation prediction from non-small cell lung cancer histopathology images using deep learning". en. In: Nat. Med. 24.10 (Oct. 2018), pp. 1559-1567.

[Cox58] D R Cox. "The Regression Analysis of Binary Sequences". In: J. R. Stat. Soc. Series B Stat. Methodol. 20.2 (1958), pp. 215-242.

[CSA19] Ashleigh Cruickshank, Geoff Stieler, and Faisal Ameer. "Evaluation of the solitary pulmonary nodule". In: Internal Medicine Journal 49.3 (2019), pp. 306-315.

[Cun+15] J J Cunningham et al. Divergent and convergent evolution in metastases suggest treatment strategies based on specific metastatic sites. 2015.

[CA20] Vesna Cuplov and Nicolas André. "Machine Learning Approach to Forecast Chemotherapy-Induced Haematological Toxicities in Patients with Rhabdomyosarcoma". In: Cancers 12.7 (2020), p. 1944. 
"Cutaneous melanoma: ESMO Clinical Practice Guidelines for diagnosis, treatment and follow-up". In: Ann. Oncol. 30.12 (Dec. 2019), pp. 1884-1901.

[Dan+16] François-Xavier Danlos et al. "Nivolumab-induced sarcoid-like granulomatous reaction in a patient with advanced melanoma". In: Chest 149.5 (2016), e133-e136.

[Del+20] Marzia Del Re et al. "A multiparametric approach to improve the prediction of response to immunotherapy in patients with metastatic NSCLC". en. In: Cancer Immunol. Immunother. (Dec. 2020).

[Del+19] Marta Della Seta et al. “A 3D quantitative imaging biomarker in pre-treatment MRI predicts overall survival after stereotactic radiation therapy of patients with a singular brain metastasis". en. In: Acta radiol. 60.11 (Nov. 2019), pp. 1496-1503.

[Dim+18] Florentia Dimitriou et al. "Sarcoid-like reactions in patients receiving modern melanoma treatment". In: Melanoma research 28.3 (2018), p. 230.

[Du+19] Yang $\mathrm{Du}$ et al. "Noninvasive imaging in cancer immunotherapy: The way to precision medicine". en. In: Cancer Lett. 466 (Dec. 2019), pp. 13-22.

[Du+18] Yue Du et al. "Classification of Tumor Epithelium and Stroma by Exploiting Image Features Learned by Deep Convolutional Neural Networks". en. In: Ann. Biomed. Eng. 46.12 (Dec. 2018), pp. 1988-1999.

[DC19] Michael J Duffy and John Crown. "Biomarkers for Predicting Response to Immunotherapy with Immune Checkpoint Inhibitors in Cancer Patients". en. In: Clin. Chem. 65.10 (Oct. 2019), pp. 1228-1238. 
[Egg+19] Hendrik Eggers et al. "Sarcoid-Like Lesions Mimicking Pulmonary Metastasis: A Case Series and Review of the Literature". In: Oncology research and treatment 42.7/8 (2019), pp. 382-386.

[Eht+17] Babak Ehteshami Bejnordi et al. "Diagnostic Assessment of Deep Learning Algorithms for Detection of Lymph Node Metastases in Women With Breast Cancer". en. In: JAMA 318.22 (Dec. 2017), pp. 2199-2210.

[Eis+09] E A Eisenhauer et al. "New response evaluation criteria in solid tumours: revised RECIST guideline (version 1.1)". en. In: Eur. J. Cancer 45.2 (Jan. 2009), pp. 228-247.

[El +14] Nagi S El Saghir et al. "Tumor boards: optimizing the structure and improving efficiency of multidisciplinary management of patients with cancer worldwide". en. In: Am Soc Clin Oncol Educ Book (2014), e461-6.

[Eri+17] Bradley Erickson et al. Data from LGG-1p19qDeletion. 2017.

[Eura] European Medicines Agency. Keytruda: EPAR - Product Information. http : / / www . ema . europa . eu / docs / en_GB / document_library / EPAR____Product _ Information / human / 003820 / WC500190990.pdf. Accessed: 2018-9-5.

[Eurb] European Medicines Agency. Opdivo: EPAR - Product Information. http : / / www . ema . europa . eu / docs / en_GB / document_library / EPAR_-_Product__ Information / human / 003985 / WC500189765 . pdf. Accessed: 2018-9-5.

[Eurc] European Medicines Agency. Yervoy. https : / / www . ema . europa . eu / en / medicines / human / EPAR / yervoy. Accessed: 2020-12-17.

[Fal+19] Thorsten Falk et al. "U-Net: deep learning for cell counting, detection, and morphometry". en. In: Nat. Methods 16.1 (Jan. 2019), pp. 67-70. 
[Fav+17] X Fave et al. "Using Pretreatment Radiomics and DeltaRadiomics Features to Predict Non-Small Cell Lung Cancer Patient Outcomes". In: Int. J. Radiat. Oncol. Biol. Phys. 98.1 (May 2017), p. 249.

[Fel15] S Feller. "One in four cancer trials fails to enroll enough participants". In: UPI. (2015).

[Fer+18] Robert L Ferris et al. "Nivolumab vs investigator's choice in recurrent or metastatic squamous cell carcinoma of the head and neck: 2-year long-term survival update of CheckMate 141 with analyses by tumor PD-L1 expression". en. In: Oral Oncol. 81 (June 2018), pp. 45-51.

[FB81] Martin A Fischler and Robert C Bolles. "Random sample consensus: a paradigm for model fitting with applications to image analysis and automated cartography". In: Commun. ACM 24.6 (June 1981), pp. 381-395.

[Fog18] David B Fogel. "Factors associated with clinical trials that fail and opportunities for improving the likelihood of success: A review". en. In: Contemp Clin Trials Commun 11 (Sept. 2018), pp. 156-164.

[Fou+13] Mona N Fouad et al. "Enrollment of patients with lung and colorectal cancers onto clinical trials". en. In: J. Oncol. Pract. 9.2 (Mar. 2013), e40-7.

[Fra+18] Daniel Franzen et al. "Ipilimumab and early signs of pulmonary toxicity in patients with metastastic melanoma: a prospective observational study". In: Cancer Immunology, Immunotherapy 67.1 (2018), pp. 127-134.

[FPP16] Claire F Friedman, Tracy A Proverbs-Singh, and Michael A Postow. "Treatment of the immune-related adverse effects of immune checkpoint inhibitors: a review". In: JAMA oncology 2.10 (2016), pp. 1346-1353. 
[Gal+20] Norbert Galldiks et al. "Imaging challenges of immunotherapy and targeted therapy in patients with brain metastases: response, progression, and pseudoprogression". en. In: Neuro. Oncol. 22.1 (Jan. 2020), pp. 17-30.

[Gam+18] T Gambichler et al. "Baseline laboratory parameters predicting clinical outcome in melanoma patients treated with ipilimumab: a single-centre analysis". In: J. Eur. Acad. Dermatol. Venereol. 32.6 (2018), pp. 972-977.

[Gan+18a] David R Gandara et al. “Blood-based tumor mutational burden as a predictor of clinical benefit in non-small-cell lung cancer patients treated with atezolizumab". In: Nat. Med. 24.9 (2018), pp. 1441-1448.

[Gan+18b] Leena Gandhi et al. "Pembrolizumab plus Chemotherapy in Metastatic Non-Small-Cell Lung Cancer". In: N. Engl. J. Med. 378.22 (Apr. 2018), pp. 2078-2092.

[Gar+19] Roberto Garcia-Figueiras et al. How clinical imaging can assess cancer biology. 2019.

[Gha+18] Sameer R Ghate et al. "Economic Burden of Adverse Events Associated with Immunotherapy and Targeted Therapy for Metastatic Melanoma in the Elderly". en. In: Am Health Drug Benefits 11.7 (Oct. 2018), pp. 334-343.

[GWA16] Geoffrey T Gibney, Louis M Weiner, and Michael B Atkins. "Predictive biomarkers for checkpoint inhibitor-based immunotherapy". en. In: Lancet Oncol. 17.12 (Dec. 2016), e542-e551.

[GKH16] Robert J Gillies, Paul E Kinahan, and Hedvig Hricak. "Radiomics: Images Are More than Pictures, They Are Data". en. In: Radiology 278.2 (Feb. 2016), pp. 563-577.

[Gol+16] Simone M Goldinger et al. "Cytotoxic cutaneous adverse drug reactions during anti-PD-1 therapy". In: Clinical Cancer Research 22.16 (2016), pp. 4023-4029. 
[Gra+16] Richard NJ Graham et al. "Return of the pulmonary nodule: the radiologist's key role in implementing the 2015 BTS guidelines on the investigation and management of pulmonary nodules". In: The British Journal of Radiology 89.1059 (2016), p. 20150776.

[GM12] Mel Greaves and Carlo C Maley. "Clonal evolution in cancer". en. In: Nature 481.7381 (Jan. 2012), pp. 306-313.

[Gre+17] Richard Green et al. "Management of pulmonary nodules in head and neck cancer patients-Our experience and interpretation of the British Thoracic Society Guidelines". In: The Surgeon 15.4 (2017), pp. 227-230.

[Gri+17a] Joost J M van Griethuysen et al. "Computational Radiomics System to Decode the Radiographic Phenotype". en. In: Cancer Res. 77.21 (Nov. 2017), e104-e107.

[Gri+17b] Joost J M van Griethuysen et al. "Computational Radiomics System to Decode the Radiographic Phenotype". en. In: Cancer Res. 77.21 (Nov. 2017), e104-e107.

[G217] J J Griethuysen - Cancer Research and 2017. "Computational Radiomics System to Decode the Radiographic Phenotype". In: (2017).

[Gro+20] Aaron Grossberg et al. HNSCC. 2020.

[Gro+17] Patrick Grossmann et al. "Defining the biological basis of radiomic phenotypes in lung cancer". en. In: Elife 6 (July 2017).

[Gru+19] Damien Gruson et al. "Data science, artificial intelligence, and machine learning: Opportunities for laboratory medicine and the value of positive regulation". In: Clin. Biochem. 69 (2019), pp. 1-7. 
[Hag+19] Akihiro Haga et al. "Standardization of imaging features for radiomics analysis". In: J. Med. Invest. 66.1.2 (2019), pp. 35-37.

[Hal+14] Darragh F Halpenny et al. "Are there imaging characteristics associated with lung adenocarcinomas harboring ALK rearrangements?" en. In: Lung Cancer 86.2 (Nov. 2014), pp. 190-194.

[Har+19] Stefan Harrer et al. "Artificial Intelligence for Clinical Trial Design". en. In: Trends Pharmacol. Sci. 40.8 (Aug. 2019), pp. 577-591.

[HKY20] Grant Haskins, Uwe Kruger, and Pingkun Yan. Deep learning in medical image registration: a survey. 2020.

[HB17] Nuh Hatipoglu and Gokhan Bilgin. "Cell segmentation in histopathological images with deep learning algorithms by utilizing spatial relationships". en. In: Med. Biol. Eng. Comput. 55.10 (Oct. 2017), pp. 1829-1848.

[HCC19a] Jonathan J Havel, Diego Chowell, and Timothy A Chan. "The evolving landscape of biomarkers for checkpoint inhibitor immunotherapy". en. In: Nat. Rev. Cancer 19.3 (Mar. 2019), pp. 133-150.

[HCC19b] Jonathan J Havel, Diego Chowell, and Timothy A Chan. "The evolving landscape of biomarkers for checkpoint inhibitor immunotherapy". en. In: Nat. Rev. Cancer 19.3 (Mar. 2019), pp. 133-150.

[Hay+77] J L Hayward et al. "Assessment of response to therapy in advanced breast cancer". en. In: Br. J. Cancer 35.3 (Mar. 1977), pp. 292-298.

[He+20] Bingxi He et al. "Predicting response to immunotherapy in advanced non-small-cell lung cancer using tumor mutational burden radiomic biomarker". In: Journal for ImmunoTherapy of Cancer 8.2 (2020), pp. 1-10. 
[He+17] Yayi He et al. "PD-1, PD-L1 Protein Expression in Non-Small Cell Lung Cancer and Their Relationship with Tumor-Infiltrating Lymphocytes". en. In: Med. Sci. Monit. 23 (Mar. 2017), pp. 1208-1216.

[Hec+20] Stefanie J Hectors et al. "MRI radiomics features predict immuno-oncological characteristics of hepatocellular carcinoma". en. In: Eur. Radiol. 30.7 (July 2020), pp. 3759 3769 .

[Hel+19] Nicholas Heller et al. C4KC KiTS Challenge Kidney Tumor Segmentation Dataset. 2019.

[Hel+18a] Matthew D Hellmann et al. "Nivolumab plus Ipilimumab in Lung Cancer with a High Tumor Mutational Burden". en. In: N. Engl. J. Med. 378.22 (May 2018), pp. 2093-2104.

[Hel+18b] Matthew D Hellmann et al. "Nivolumab plus Ipilimumab in Lung Cancer with a High Tumor Mutational Burden". In: N. Engl. J. Med. 378.22 (2018), pp. 2093-2104.

[Hen+17] Shona Hendry et al. "Assessing Tumor-infiltrating Lymphocytes in Solid Tumors: A Practical Review for Pathologists and Proposal for a Standardized Method From the International Immunooncology Biomarkers Working Group". In: Adv. Anat. Pathol. 24.5 (2017), pp. 235-251.

[HGM17] Carl Heneghan, Ben Goldacre, and Kamal R Mahtani. "Why clinical trial outcomes fail to translate into benefits for patients". en. In: Trials 18.1 (Mar. 2017), p. 122.

[Hen+02] Claudia I Henschke et al. "CT screening for lung cancer: frequency and significance of part-solid and nonsolid nodules". In: American Journal of Roentgenology 178.5 (2002), pp. 1053-1057. 
[Her+16] Roy S Herbst et al. "Pembrolizumab versus docetaxel for previously treated, PD-L1-positive, advanced non-small-cell lung cancer (KEYNOTE-010): a randomised controlled trial". en. In: Lancet 387.10027 (Apr. 2016), pp. 1540-1550.

[Hir+05] Takashi Hirose et al. "Patients preferences in chemotherapy for advanced non-small-cell lung cancer". en. In: Intern. Med. 44.2 (Feb. 2005), pp. 107-113.

[Ho+20a] Tsung-Ying Ho et al. “Classifying Neck Lymph Nodes of Head and Neck Squamous Cell Carcinoma in MRI Images with Radiomic Features". en. In: J. Digit. Imaging (Jan. 2020).

[Ho+20b] Won Jin Ho et al. Multipanel mass cytometry reveals antiPD-1 therapy-mediated $B$ and $T$ cell compartment remodeling in tumor-draining lymph nodes. 2020.

[Hod+10] F Stephen Hodi et al. "Improved survival with ipilimumab in patients with metastatic melanoma". In: New England Journal of Medicine 363.8 (2010), pp. 711-723.

[Hof19] Paul Hofman. "The challenges of evaluating predictive biomarkers using small biopsy tissue samples and liquid biopsies from non-small cell lung cancer patients". en. In: J. Thorac. Dis. 11.Suppl 1 (Jan. 2019), S57-S64.

[Hos+18] Ahmed Hosny et al. "Artificial intelligence in radiology". en. In: Nat. Rev. Cancer (May 2018).

[HMM19] Masatoshi Hotta, Ryogo Minamimoto, and Kenta Miwa. "11C-methionine-PET for differentiating recurrent brain tumor from radiation necrosis: radiomics approach with random forest classifier". en. In: Sci. Rep. 9.1 (Oct. 2019), p. 15666.

[Hri11] Hedvig Hricak. "Oncologic imaging: a guiding hand of personalized cancer care". en. In: Radiology 259.3 (June 2011), pp. 633-640. 
[Hua+13] Yuhui Huang et al. "Vascular normalization as an emerging strategy to enhance cancer immunotherapy". en. In: Cancer Res. 73.10 (May 2013), pp. 2943-2948.

[Hwa+16] Thomas J Hwang et al. "Failure of Investigational Drugs in Late-Stage Clinical Development and Publication of Trial Results". en. In: JAMA Intern. Med. 176.12 (Dec. 2016), pp. 1826-1833.

[IRT07] MC Iannuzzi, BA Rybicki, and AS Teirstein. "Sarcoidosis". In: J Engl J Med 357 (2007), pp. 2153-2165.

[Ion+93] Y Ionov et al. "Ubiquitous somatic mutations in simple repeated sequences reveal a new mechanism for colonic carcinogenesis". en. In: Nature 363.6429 (June 1993), pp. 558561.

[Ish+92] Y Ishida et al. "Induced expression of PD-1, a novel member of the immunoglobulin gene superfamily, upon programmed cell death". en. In: EMBO J. 11.11 (Nov. 1992), pp. 3887-3895.

[JM] Mika S Jain and Tarik F Massoud. Predicting tumour mutational burden from histopathological images using multiscale deep learning.

[Joh+16] Douglas B Johnson et al. "Fulminant Myocarditis with Combination Immune Checkpoint Blockade". en. In: N. Engl. J. Med. 375.18 (Nov. 2016), pp. 1749-1755.

[JPP18] Dennis Jones, Ethel R Pereira, and Timothy P Padera. "Growth and Immune Evasion of Lymph Node Metastasis". en. In: Front. Oncol. 8 (Feb. 2018), p. 36.

[JD19] Shweta Joshi and Donald L Durden. "Combinatorial Approach to Improve Cancer Immunotherapy: Rational Drug Design Strategy to Simultaneously Hit Multiple Targets to Kill Tumor Cells and to Activate the Immune System". en. In: J. Oncol. 2019 (Feb. 2019), p. 5245034. 
[Jua+18] Tiffany M Juarez et al. "Understanding the brain tumor microenvironment: Considerations to applying systems biology and immunotherapy". en. In: International Journal of Neurooncology 1.1 (Jan. 2018), p. 25.

[Kat+19] Jakob Nikolas Kather et al. “Deep learning can predict microsatellite instability directly from histology in gastrointestinal cancer". en. In: Nat. Med. 25.7 (July 2019), pp. 1054-1056.

[Kel+19] Christopher J Kelly et al. "Key challenges for delivering clinical impact with artificial intelligence". en. In: BMC Med. 17.1 (Oct. 2019), p. 195.

[Ker+15] Keith M Kerr et al. "Programmed Death-Ligand 1 Immunohistochemistry in Lung Cancer: In what state is this art?" en. In: J. Thorac. Oncol. 10.7 (July 2015), pp. 985-989.

[Kho+20] Mohammadhadi Khorrami et al. "Changes in CT radiomic features associated with lymphocyte distribution predict overall survival and response to immunotherapy in non-small cell lung cancer". In: Cancer Immunology Research 8.1 (2020), pp. 108-119.

[Kic+16] Philipp Kickingereder et al. "Radiomic Profiling of Glioblastoma: Identifying an Imaging Predictor of Patient Survival with Improved Performance over Established Clinical and Radiologic Risk Models". en. In: Radiology 280.3 (Sept. 2016), pp. 880-889.

[Kim+13] Sae Byol Kim et al. "Ground-glass opacity in lung metastasis from breast cancer: a case report". In: Tuberculosis and Respiratory Diseases 74.1 (2013), pp. 32-36.

[Kim19] Kwang Gi Kimg. “Deep Learning”. In: Healthc. Inform. Res. 22.4 (2019), pp. 351-354.

[Kin+17] Paul Kinahan et al. Data from ACRIN-FLT-Breast. 2017.

[Kin+18] Paul Kinahan et al. Data from ACRIN-FMISO-Brain. 2018. 
[Kin+19] Paul Kinahan et al. Data from the ACRIN 6668 Trial NSCLCFDG-PET. 2019.

[KB14] Diederik P Kingma and Jimmy Ba. "Adam: A method for stochastic optimization". In: arXiv preprint arXiv:1412.6980 (2014).

[Kir+17] Margarita Kirienko et al. "Prediction of disease-free survival by the PET/CT radiomic signature in non-small cell lung cancer patients undergoing surgery". en. In: Eur. J. Nucl. Med. Mol. Imaging (Sept. 2017).

[Kis+16] Pavel Kisilev et al. "Semantic description of medical image findings: structured learning approach". In: Proceedings of the Britisch Machine Vision Conference (2016), pp. 171.1-171.11.

[Kle+10] Stefan Klein et al. "elastix: a toolbox for intensity-based medical image registration". en. In: IEEE Trans. Med. Imaging 29.1 (Jan. 2010), pp. 196-205.

[Kni+19] Helge C Kniep et al. "Radiomics of Brain MRI: Utility in Prediction of Metastatic Tumor Type". en. In: Radiology 290.2 (Feb. 2019), pp. 479-487.

[Al-+18] Yousef Al-Kofahi et al. "A deep learning-based algorithm for 2-D cell segmentation in microscopy images". en. In: BMC Bioinformatics 19.1 (Oct. 2018), p. 365.

[KJ14] Michael D Kuo and Neema Jamshidi. "Behind the numbers: Decoding molecular phenotypes with radiogenomics- guiding principles and technical considerations". In: Radiology 270.2 (2014), pp. 320-325.

[Kur+15] Karen A Kurdziel et al. Data From NaF PROSTATE. 2015.

[Lar+18] James Larkin et al. “Overall Survival in Patients With Advanced Melanoma Who Received Nivolumab Versus Investigator's Choice Chemotherapy in CheckMate 037: A Randomized, Controlled, Open-Label Phase III Trial". en. In: J. Clin. Oncol. 36.4 (Feb. 2018), pp. 383-390. 
[LKA96] D R Leach, M F Krummel, and J P Allison. "Enhancement of antitumor immunity by CTLA-4 blockade". en. In: Science 271.5256 (Mar. 1996), pp. 1734-1736.

[Li+16] Hui Li et al. "MR Imaging Radiomics Signatures for Predicting the Risk of Breast Cancer Recurrence as Given by Research Versions of MammaPrint, Oncotype DX, and PAM50 Gene Assays". en. In: Radiology 281.2 (Nov. 2016), pp. 382-391.

[Li+20] Menglei Li et al. "A clinical-radiomics nomogram for the preoperative prediction of lymph node metastasis in colorectal cancer". en. In: J. Transl. Med. 18.1 (Jan. 2020), p. 46.

[Li+18] Qian $\mathrm{Li}$ et al. "Comparison Between Radiological Semantic Features and Lung-RADS in Predicting Malignancy of Screen-Detected Lung Nodules in the National Lung Screening Trial". en. In: Clin. Lung Cancer 19.2 (Mar. 2018), 148-156.e3.

[Lib+11] Arthur Liberzon et al. "Molecular signatures database (MSigDB) 3.0". en. In: Bioinformatics 27.12 (June 2011), pp. 1739-1740.

[Lin+17] Tsung-Yi Lin et al. "Focal loss for dense object detection". In: Proceedings of the IEEE international conference on computer vision. 2017, pp. 2980-2988.

[LG16] Helena Linardou and Helen Gogas. "Toxicity management of immunotherapy for patients with metastatic melanoma". In: Annals of translational medicine 4.14 (2016).

[Liu+20] Xiaoxuan Liu et al. Reporting guidelines for clinical trial reports for interventions involving artificial intelligence: the CONSORT-AI extension. 2020. 
[Liu+19] Zhenyu Liu et al. "The Applications of Radiomics in Precision Diagnosis and Treatment of Oncology: Opportunities and Challenges". en. In: Theranostics 9.5 (Feb. 2019), pp. 1303-1322.

[Loh+18] Philipp Lohmann et al. "Combined FET PET/MRI radiomics differentiates radiation injury from recurrent brain metastasis". en. In: Neuroimage Clin 20 (Aug. 2018), pp. 537-542.

[Lov+18] Pierre Lovinfosse et al. "FDG PET/CT radiomics for predicting the outcome of locally advanced rectal cancer". In: European Journal of Nuclear Medicine and Molecular Imaging 45.3 (2018), pp. 365-375.

[LN19] Siew-Kee Low and Yusuke Nakamura. "The road map of cancer precision medicine with the innovation of advanced cancer detection technology and personalized immunotherapy". en. In: Jpn. J. Clin. Oncol. 49.7 (July 2019), pp. 596-603.

[Lu+19] Victor $\mathrm{M} \mathrm{Lu}$ et al. "Concurrent versus non-concurrent immune checkpoint inhibition with stereotactic radiosurgery for metastatic brain disease: a systematic review and meta-analysis". In: J. Neurooncol. 141.1 (Jan. 2019), pp. 1-12.

[Luc+19] C Luchini et al. "ESMO recommendations on microsatellite instability testing for immunotherapy in cancer, and its relationship with PD-1/PD-L1 expression and tumour mutational burden: a systematic review-based approach". en. In: Ann. Oncol. 30.8 (Aug. 2019), pp. 1232-1243.

[Lüt+20] Susanne Lütje et al. "Immune Checkpoint Imaging in Oncology: A Game Changer Toward Personalized Immunotherapy?" en. In: J. Nucl. Med. 61.8 (Aug. 2020), pp. 1137-1144. 
[Lv+18] J Lv et al. "Comparison of CT radiogenomic and clinical characteristics between EGFR and KRAS mutations in lung adenocarcinomas". en. In: Clin. Radiol. 73.6 (June 2018), 590.e1-590.e8.

[Lyl+16] L T Lyle et al. “Alterations in pericyte subpopulations are associated with elevated blood-tumor barrier permeability in experimental brain metastasis of breast cancer". In: Clin. Cancer Res. (2016).

[Ma+16] Weijie Ma et al. "Current status and perspectives in translational biomarker research for PD-1/PD-L1 immune checkpoint blockade therapy". en. In: J. Hematol. Oncol. 9.1 (May 2016), p. 47.

[MSG17] Laura Maciejko, Munisha Smalley, and Aaron Goldman. "Cancer Immunotherapy and Personalized Medicine: Emerging Technologies and Biomarker-Based Approaches". en. In: J. Mol. Biomark. Diagn. 8.5 (Sept. 2017).

[Mah+19] Faisal Mahmood et al. “Deep Adversarial Training for Multi-Organ Nuclei Segmentation in Histopathology Images". en. In: IEEE Trans. Med. Imaging PP (July 2019).

[MK16] Artem B Mamonov and Jayashree Kalpathy-Cramer. Data From QIN GBM Treatment Response. 2016.

[Man+13] Subramani Mani et al. "Machine learning for predicting the response of breast cancer to neoadjuvant chemotherapy". In: J. Am. Med. Inform. Assoc. 20.4 (2013), pp. 688695.

[Man11] Matthew Mansh. "Ipilimumab and cancer immunotherapy: a new hope for advanced stage melanoma". In: The Yale journal of biology and medicine 84.4 (2011), p. 381. 
[Mar+19] Leigh Marcus et al. "FDA approval summary: pembrolizumab for the treatment of microsatellite instability-high solid tumors". In: Clinical Cancer Research 25.13 (2019), pp. 3753-3758.

[Mar+14] Guilherme Nader Marta et al. "Treatment priorities in oncology: do we want to live longer or better?" en. In: Clinics 69.8 (Aug. 2014), pp. 509-514.

[May+20] Marius E Mayerhoefer et al. "Introduction to radiomics". In: J. Nucl. Med. 61.4 (2020), pp. 488-495.

[Maz+19] Maciej A Mazurowski et al. “Deep learning in radiology: An overview of the concepts and a survey of the state of the art with focus on MRI". In: J. Magn. Reson. Imaging 49.4 (2019), pp. 939-954.

[Maz+20] Giulia Mazzaschi et al. "Integrated CT imaging and tissue immune features disclose a radio-immune signature with high prognostic impact on surgically resected NSCLC". en. In: Lung Cancer 144 (June 2020), pp. 30-39.

[McG+16] Nicholas McGranahan et al. "Clonal neoantigens elicit $\mathrm{T}$ cell immunoreactivity and sensitivity to immune checkpoint blockade". en. In: Science 351.6280 (Mar. 2016), pp. 1463-1469.

[McK+20] William B McKean et al. "Biomarkers in Precision Cancer Immunotherapy: Promise and Challenges". en. In: Am Soc Clin Oncol Educ Book 40 (May 2020), e275-e291.

[McN+07] M F McNitt-Gray et al. The Lung Image Database Consortium (LIDC) data collection process for nodule detection and annotation. 2007.

[McQ+95] R P McQuellon et al. "Patient preferences for treatment of metastatic breast cancer: a study of women with earlystage breast cancer". en. In: J. Clin. Oncol. 13.4 (Apr. 1995), pp. 858-868. 
[Mek+18] Ahmed Mekki et al. "Detection of immune-related adverse events by medical imaging in patients treated with anti-programmed cell death 1". en. In: Eur. J. Cancer 96 (June 2018), pp. 91-104.

[Men+15] Xiangjiao Meng et al. "Predictive biomarkers in PD-1/PD-L1 checkpoint blockade immunotherapy". en. In: Cancer Treat. Rev. 41.10 (Dec. 2015), pp. 868-876.

[Mic+19] O Michielin et al. “Cutaneous melanoma: ESMO Clinical Practice Guidelines for diagnosis, treatment and followup". In: Ann. Oncol. 30.12 (2019), pp. 1884-1901.

[Al-+19] Zahraa Al-Milaji et al. Integrating segmentation with deep learning for enhanced classification of epithelial and stromal tissues in HEE images. 2019.

[MS05] Wallace T Miller Jr and Rosita M Shah. "Isolated diffuse ground-glass opacity in thoracic CT: causes and clinical presentations". In: American Journal of Roentgenology 184.2 (2005), pp. 613-622.

[Möl+20] Miriam Möller et al. "Blood Immune Cell Biomarkers in Patient With Lung Cancer Undergoing Treatment With Checkpoint Blockade". In: J. Immunother. 43.2 (2020), pp. 57-66.

[Mot+19] Robert J Motzer et al. “Nivolumab plus ipilimumab versus sunitinib in first-line treatment for advanced renal cell carcinoma: extended follow-up of efficacy and safety results from a randomised, controlled, phase 3 trial". en. In: Lancet Oncol. 20.10 (Oct. 2019), pp. 1370-1385.

[Mot+20] Robert J Motzer et al. "Survival outcomes and independent response assessment with nivolumab plus ipilimumab versus sunitinib in patients with advanced renal cell carcinoma: 42-month follow-up of a randomized phase 3 clinical trial". en. In: J Immunother Cancer 8.2 (July 2020). 
[Mu+18] Wei $\mathrm{Mu}$ et al. Radiomic biomarkers from PET/CT multi-modality fusion images for the prediction of immunotherapy response in advanced non-small cell lung cancer patients. 2018.

[Mu+20] Wei $\mathrm{Mu}$ et al. "Radiomics of 18F-FDG PET/CT images predicts clinical benefit of advanced NSCLC patients to checkpoint blockade immunotherapy". en. In: Eur. J. Nucl. Med. Mol. Imaging 47.5 (May 2020), pp. 1168-1182.

[MWK15] Peter Muzi, Michelle Wanner, and Paul Kinahan. Data From RIDER Lung PET-CT. 2015.

[Nai+18] Arjun Nair et al. "Variable radiological lung nodule evaluation leads to divergent management recommendations". In: European Respiratory Journal 52.6 (2018).

[Nar+20] Valerio Nardone et al. "Radiomics predicts survival of patients with advanced non-small cell lung cancer undergoing PD-1 blockade using Nivolumab". en. In: Oncol. Lett. 19.2 (Feb. 2020), pp. 1559-1566.

[NO19] Faria Nasim and David E Ost. "Management of the solitary pulmonary nodule". In: Current opinion in pulmonary medicine 25.4 (2019), pp. 344-353.

[Nay+17] Peter Naylor et al. Nuclei segmentation in histopathology images using deep neural networks. 2017.

[Ner+20] Emanuele Neri et al. "Involvement of radiologists in oncologic multidisciplinary team meetings: an international survey by the European Society of Oncologic Imaging". en. In: Eur. Radiol. (Aug. 2020).

[Nie+19] Ke Nie et al. "NCTN Assessment on Current Applications of Radiomics in Oncology". en. In: Int. J. Radiat. Oncol. Biol. Phys. 104.2 (June 2019), pp. 302-315. 
[NHH19] Mizuki Nishino, Hiroto Hatabu, and F Stephen Hodi. "Imaging of Cancer Immunotherapy: Current Approaches and Future Directions". en. In: Radiology 290.1 (Jan. 2019), pp. 9-22.

[Nis+15] Mizuki Nishino et al. "Cancer immunotherapy and immune-related response assessment: the role of radiologists in the new arena of cancer treatment". In: European journal of radiology 84.7 (2015), pp. 1259-1268.

[Nis+13] Mizuki Nishino et al. "Developing a common language for tumor response to immunotherapy: immune-related response criteria using unidimensional measurements". en. In: Clin. Cancer Res. 19.14 (July 2013), pp. 3936-3943.

[OCo+17] James P B O'Connor et al. "Imaging biomarker roadmap for cancer studies". en. In: Nat. Rev. Clin. Oncol. 14.3 (Mar. 2017), pp. 169-186.

[Ohs+17] Shinichiro Ohshimo et al. "Differential diagnosis of granulomatous lung disease: clues and pitfalls: Number 4 in the Series "Pathology for the clinician" Edited by Peter Dorfmüller and Alberto Cavazza". In: European Respiratory Review 26.145 (2017), p. 170012.

[Oka+15] Hideho Okada et al. "Immunotherapy response assessment in neuro-oncology: a report of the RANO working group". en. In: Lancet Oncol. 16.15 (Nov. 2015), e534-e542.

[Ols+16] Randal S Olson et al. "Evaluation of a Tree-based Pipeline Optimization Tool for Automating Data Science". In: Proceedings of the Genetic and Evolutionary Computation Conference 2016. GECCO '16. Denver, Colorado, USA: ACM, 2016, pp. 485-492.

[Opi+20] Mark P van Opijnen et al. "The impact of current treatment modalities on the outcomes of patients with melanoma brain metastases: A systematic review". en. In: Int. J. Cancer 146.6 (Mar. 2020), pp. 1479-1489. 
[OGB20] Ohad Oren, Bernard J Gersh, and Deepak L Bhatt. "Artificial intelligence in medical imaging: switching from radiographic pathological data to clinically meaningful endpoints". In: The Lancet Digital Health 2.9 (2020), e486-e488.

[Org+79] World Health Organization et al. WHO handbook for reporting results of cancer treatment. World Health Organization, 1979.

[Ort+17] R Ortiz-Ramón et al. "A radiomics evaluation of 2D and 3D MRI texture features to classify brain metastases from lung cancer and melanoma". In: 2017 39th Annual International Conference of the IEEE Engineering in Medicine and Biology Society (EMBC). July 2017, pp. 493-496.

[Ort+18] Rafael Ortiz-Ramón et al. "Classifying brain metastases by their primary site of origin using a radiomics approach based on texture analysis: a feasibility study". en. In: Eur. Radiol. 28.11 (Nov. 2018), pp. 4514-4523.

[Ove+13] Michael J Overman et al. "Use of research biopsies in clinical trials: are risks and benefits adequately discussed?" en. In: J. Clin. Oncol. 31.1 (Jan. 2013), pp. 17-22.

[Pai+19] Sara I Pai et al. "Comparative analysis of the phase III clinical trials of anti-PD1 monotherapy in head and neck squamous cell carcinoma patients (CheckMate 141 and KEYNOTE 040)". en. In: J Immunother Cancer 7.1 (Apr. 2019), p. 96.

[PGW95] HS Pandha, H Griffiths, and J Waxman. "Sarcoidosis and cancer". In: Clinical Oncology 7.5 (1995), pp. 277-278.

[PMK20] Nikolaos Papanikolaou, Celso Matos, and Dow Mu Koh. "How to develop a meaningful radiomic signature for clinical use in oncologic patients". In: Cancer Imaging 20.1 (2020), pp. 1-10. 
[Par+20a] Changhee Park et al. "Tumor immune profiles noninvasively estimated by FDG PET with deep learning correlate with immunotherapy response in lung adenocarcinoma". en. In: Theranostics 10.23 (Aug. 2020), pp. 10838-10848.

[Par+05] JES Park et al. "The HRCT appearances of granulomatous pulmonary disease in common variable immune deficiency". In: European journal of radiology 54.3 (2005), pp. 359-364.

[Par+20b] Kye Jin Park et al. "Radiomics-based prediction model for outcomes of PD-1/PD-L1 immunotherapy in metastatic urothelial carcinoma". en. In: Eur. Radiol. 30.10 (Oct. 2020), pp. 5392-5403.

[Par+20c] Kye Jin Park et al. "Radiomics-based prediction model for outcomes of PD-1/PD-L1 immunotherapy in metastatic urothelial carcinoma". en. In: Eur. Radiol. 30.10 (Oct. 2020), pp. 5392-5403.

[Par+15] Chintan Parmar et al. "Radiomic Machine-Learning Classifiers for Prognostic Biomarkers of Head and Neck Cancer". en. In: Front. Oncol. 5 (Dec. 2015), p. 272.

[PU19] Luigi Pasini and Paola Ulivi. "Liquid Biopsy for the Detection of Resistance Mechanisms in NSCLC: Comparison of Different Blood Biomarkers". In: J. Clin. Med. Res. 8.7 (2019), p. 998.

[Pat+19] Pradnya Patil et al. A combination of intra- and peritumoral features on baseline CT scans is associated with overall survival in non-small cell lung cancer patients treated with immune checkpoint inhibitors: a multi-agent multi-site study. 2019.

[PPT19a] Madhavi Patnana, Sapna Patel, and Anne Tsao. Anti-PD-1 Immunotherapy Melanoma Dataset. 2019.

[PPT19b] Madhavi Patnana, Sapna Patel, and Anne S Tsao. Data from Anti-PD-1 Immunotherapy Lung. 2019. 
[Pee+18] Jan C Peeken et al. "Semantic imaging features predict disease progression and survival in glioblastoma multiforme patients". In: Strahlenther. Onkol. 194.6 (2018), pp. 580-590.

[Pen20] Lihong Peng. "Peripheral blood markers predictive of outcome and immune-related adverse events in advanced non-small cell lung cancer treated with PD-1 inhibitors". In: Cancer Immunol. Immunother. (2020), p. 10.

[Pen+18] Luke Peng et al. "Distinguishing True Progression From Radionecrosis After Stereotactic Radiation Therapy for Brain Metastases With Machine Learning and Radiomics". In: International Journal of Radiation Oncology*Biology*Physics 102.4 (Nov. 2018), pp. 1236-1243.

[Pet+19] Fausto Petrelli et al. "Combination of radiotherapy and immunotherapy for brain metastases: A systematic review and meta-analysis". en. In: Crit. Rev. Oncol. Hematol. 144 (Dec. 2019), p. 102830.

[Pet+] Mila P Petrova et al. "Neutrophil to lymphocyte ratio as a potential predictive marker for treatment with pembrolizumab as a second line treatment in patients with non-small cell lung cancer". In: Biosci. Trends (), p. 8.

[PCW19] Sean P Pitroda, Steven J Chmura, and Ralph R Weichselbaum. "Integration of radiotherapy and immunotherapy for treatment of oligometastases". en. In: Lancet Oncol. 20.8 (Aug. 2019), e434-e442.

[Pla+18] D Planchard et al. "Metastatic non-small cell lung cancer: ESMO Clinical Practice Guidelines for diagnosis, treatment and follow-up". en. In: Ann. Oncol. 29.Suppl 4 (Oct. 2018), pp. iv192-iv237. 
[Pol+20] Giulia Polverari et al. "18F-FDG Pet Parameters and Radiomics Features Analysis in Advanced Nsclc Treated with Immunotherapy as Predictors of Therapy Response and Survival". en. In: Cancers 12.5 (May 2020).

[Pop+05] Whitney B Pope et al. "MR imaging correlates of survival in patients with high-grade gliomas". In: AJNR Am. J. Neuroradiol. 26.10 (2005), pp. 2466-2474.

[PT17] Zoran B Popovic and James D Thomas. "Assessing observer variability: A user's guide". In: Cardiovascular Diagnosis and Therapy 7.3 (2017), pp. 317-324.

[Pra+16] Prateek Prasanna et al. "Radiomic features from the peritumoral brain parenchyma on treatment-nave multi-parametric MR imaging predict long versus short-term survival in glioblastoma multiforme: Preliminary findings". en. In: Eur. Radiol. (Oct. 2016).

[Qia+19] Zenghui Qian et al. "Differentiation of glioblastoma from solitary brain metastases using radiomic machine-learning classifiers". en. In: Cancer Lett. 451 (June 2019), pp. 128-135.

[Rah+19] Paul Rahul et al. "Explaining Deep Features Using Radiologist-Defined Semantic Features and Traditional Quantitative Features". In: Tomography (Ann Arbor, Mich. ) 5.1 (2019), pp. 192-200.

[Ram+20] Santiago Ramón y Cajal et al. "Clinical implications of intratumor heterogeneity: challenges and opportunities". In: J. Mol. Med. 98.2 (Feb. 2020), pp. 161-177.

[Ran+18] Aman Rana et al. Computational Histological Staining and Destaining of Prostate Core Biopsy RGB Images with Generative Adversarial Neural Networks. 2018. 
[Rec+20] Michael P Recht et al. "Integrating artificial intelligence into the clinical practice of radiology: challenges and recommendations". In: Eur. Radiol. 30.6 (2020), pp. 3576-3584.

[Rei06] Jerome M Reich. "Neoplasia in the etiology of sarcoidosis". In: European Journal of Internal Medicine 17.2 (2006), pp. 81-87.

[Rid+16] Carole A Ridge et al. "Differentiating between subsolid and solid pulmonary nodules at CT: Inter- and intraobserver agreement between experienced thoracic radiologists". In: Radiology 278.3 (2016), pp. 888-896.

[Rio+17] Emmanuel Rios Velazquez et al. "Somatic Mutations Drive Distinct Imaging Phenotypes in Lung Cancer". en. In: Cancer Res. 77.14 (July 2017), pp. 3922-3930.

[Riv+20] Samantha Cruz Rivera et al. Guidelines for clinical trial protocols for interventions involving artificial intelligence: the SPIRIT-AI extension. 2020.

[Riz+15] Naiyer A Rizvi et al. "Cancer immunology. Mutational landscape determines sensitivity to PD-1 blockade in non-small cell lung cancer". en. In: Science 348.6230 (Apr. 2015), pp. 124-128.

[Riz+16] Stefania Rizzo et al. "CT Radiogenomic Characterization of EGFR, K-RAS, and ALK Mutations in Non-Small Cell Lung Cancer". en. In: Eur. Radiol. 26.1 (Jan. 2016), pp. 32 42.

[Riz+18] Stefania Rizzo et al. "Radiomics: the facts and the challenges of image analysis". In: European Radiology Experimental 2.1 (2018).

[Roc+19] Danilo Rocco et al. "The role of combination chemo-immunotherapy in advanced non-small cell lung cancer". en. In: Expert Rev. Anticancer Ther. 19.7 (July 2019), pp. 561-568. 
[Rog+20] William Rogers et al. "Radiomics: from qualitative to quantitative imaging". In: Br. J. Radiol. 93.1108 (Apr. 2020), p. 20190948.

[RFB15] Olaf Ronneberger, Philipp Fischer, and Thomas Brox. "U-Net: Convolutional Networks for Biomedical Image Segmentation". en. In: Medical Image Computing and Computer-Assisted Intervention - MICCAI 2015. Springer, Cham, Oct. 2015, pp. 234-241.

[Ros+19] Javier Ros et al. Review of immunogenomics and the role of tumor mutational burden as a biomarker for immunotherapy response. 2019.

[Ros+15] Andrew B Rosenkrantz et al. "Clinical utility of quantitative imaging". en. In: Acad. Radiol. 22.1 (Jan. 2015), pp. 3349.

[Ros+18] Samuel Rosner et al. "Peripheral blood clinical laboratory variables associated with outcomes following combination nivolumab and ipilimumab immunotherapy in melanoma". In: Cancer Med. 7.3 (2018), pp. 690-697.

[Rot+15] Holger Roth et al. A new 2.5 D representation for lymph node detection in CT. 2015.

[Rul+19] Eliana Rulli et al. The impact of targeted therapies and immunotherapy in melanoma brain metastases: A systematic review and meta-analysis. 2019.

[Run+19] Francesco Rundo et al. "Advanced deep learning embedded motion radiomics pipeline for predicting anti-PD-1/PD-L1 immunotherapy response in the treatment of bladder cancer: Preliminary results". In: Electronics (Switzerland) 8.10 (2019).

[RN03] Stuart Jonathan Russell and Peter Norvig. Artificial Intelligence: A Modern Approach. en. Prentice Hall/Pearson Education, 2003. 
[RK09] Aaron M Rutman and Michael D Kuo. "Radiogenomics: Creating a link between molecular diagnostics and diagnostic imaging". In: Eur. J. Radiol. 70.2 (2009), pp. 232-241.

[Sab+19] Nabil F Saba et al. "Nivolumab versus investigator's choice in patients with recurrent or metastatic squamous cell carcinoma of the head and neck: Efficacy and safety in CheckMate 141 by age". en. In: Oral Oncol. 96 (Sept. 2019), pp. 7-14.

[Sal+18] Joel Saltz et al. "Spatial Organization and Molecular Correlation of Tumor-Infiltrating Lymphocytes Using Deep Learning on Pathology Images". en. In: Cell Rep. 23.1 (Apr. 2018), 181-193.e7.

[SC18] Christine R Sanderson and David C Currow. "Palliative care meets immunotherapy: what happens as cancer paradigms change?" en. In: BMJ Support. Palliat. Care 8.4 (Dec. 2018), pp. 431-432.

[Sca+19] Lisa Scarpace et al. Data From REMBRANDT. 2019.

[SP19] Kathleen Schmainda and Melissa Prah. Data from BrainTumor-Progression. 2019.

[SS16] Ton N Schumacher and Wouter Scheper. "A liquid biopsy for cancer immunotherapy". en. In: Nat. Med. 22.4 (Apr. 2016), pp. 340-341.

[Sch+16] Lawrence H Schwartz et al. "RECIST 1.1-Update and clarification: From the RECIST committee". In: Eur. J. Cancer 62 (July 2016), pp. 132-137.

[Seg+07] Eran Segal et al. "Decoding global gene expression programs in liver cancer by noninvasive imaging". In: Nature biotechnology 25.6 (2007), pp. 675-680.

[Sev+17] Eva M Sevick-Muraca et al. "Moonshot acceleration factor: medical imaging". In: Cancer Research 77.21 (2017), pp. 5717-5720. 
[Sey+17a] Lesley Seymour et al. iRECIST: guidelines for response criteria for use in trials testing immunotherapeutics. 2017.

[Sey+17b] Lesley Seymour et al. iRECIST: guidelines for response criteria for use in trials testing immunotherapeutics. 2017.

[Sha+19] Lingdao Sha et al. "Multi-Field-of-View Deep Learning Model Predicts Nonsmall Cell Lung Cancer Programmed Death-Ligand 1 Status from Whole-Slide Hematoxylin and Eosin Images". en. In: J. Pathol. Inform. 10 (July 2019), p. 24.

[Sha+20] Xue Sha et al. "Discrimination of mediastinal metastatic lymph nodes in NSCLC based on radiomic features in different phases of CT imaging". en. In: BMC Med. Imaging 20.1 (Feb. 2020), p. 12.

[Sha+16] Nameeta Shah et al. Data from Ivy GAP. 2016.

[Sha+13] Denis P Shamonin et al. "Fast parallel image registration on CPU and GPU for diagnostic classification of Alzheimer's disease". en. In: Front. Neuroinform. 7 (2013), p. 50.

[Shi+15] Brianh Shirts et al. "Clinical laboratory analytics: Challenges and promise for an emerging discipline". In: J. Pathol. Inform. 6.1 (2015), p. 9.

[Sid+17] Parveen Sidhu et al. "Radiological manifestations of immune-related adverse effects observed in patients with melanoma undergoing immunotherapy". In: Journal of Medical Imaging and Radiation Oncology 61.6 (2017), pp. 759-766.

[SVZ13] Karen Simonyan, Andrea Vedaldi, and Andrew Zisserman. "Deep inside convolutional networks: Visualising image classification models and saliency maps". In: arXiv preprint arXiv:1312.6034 (2013). 
[Sir+16] Korsuk Sirinukunwattana et al. "Locality Sensitive Deep Learning for Detection and Classification of Nuclei in Routine Colon Cancer Histology Images". en. In: IEEE Trans. Med. Imaging 35.5 (May 2016), pp. 1196-1206.

[Siu+17] L L Siu et al. "Challenges and opportunities in adapting clinical trial design for immunotherapies". In: (2017).

[Sle+90] M L Slevin et al. "Attitudes to chemotherapy: comparing views of patients with cancer with those of doctors, nurses, and general public". en. In: BMJ 300.6737 (June 1990), pp. 1458-1460.

[Smi+20] Annabel Smith et al. "Duration of immunotherapy should we continue ad infinitum?" en. In: Intern. Med. J. 50.7 (July 2020), pp. 865-868.

[Smi15] Clark K Smith K. Data From CT_COLONOGRAPHY. 2015.

[SMH19] Alexandra Snyder, Michael P Morrissey, and Matthew D Hellmann. "Use of Circulating Tumor DNA for Cancer Immunotherapy". en. In: Clin. Cancer Res. 25.23 (Dec. 2019), pp. 6909-6915.

[Soy+18] Aixa E Soyano et al. "Peripheral blood biomarkers correlate with outcomes in advanced non-small cell lung Cancer patients treated with anti-PD-1 antibodies". In: Journal for ImmunoTherapy of Cancer 6.1 (2018), p. 129.

[SDL16] Lavinia Spain, Stefan Diem, and James Larkin. "Management of toxicities of immune checkpoint inhibitors". In: Cancer treatment reviews 44 (2016), pp. 51-60.

[SM19] Mary H Stanfill and David T Marc. "Health Information Management: Implications of Artificial Intelligence on Healthcare Data and Information Management". en. In: Yearb. Med. Inform. 28.1 (Aug. 2019), pp. 56-64. 
[Ste+21] Stefano Trebeschi, Thi Dan Linh Nguyen-Kim et al. "AIdriven identification of prognostic response patterns to immunotherapy of melanoma brain metastases". In: Submitted for publication. (2021).

[Ste+20] Julie E Stein et al. Pan-Tumor Pathologic Scoring of Response to PD-(L)1 Blockade. 2020.

[Sub+05] Aravind Subramanian et al. "Gene set enrichment analysis: a knowledge-based approach for interpreting genome-wide expression profiles". en. In: Proc. Natl. Acad. Sci. U. S. A. 102.43 (Oct. 2005), pp. 15545-15550.

[Sun+20a] Kai-Yu Sun et al. "CT-based radiomics scores predict response to neoadjuvant chemotherapy and survival in patients with gastric cancer". en. In: BMC Cancer 20.1 (May 2020), p. 468.

[Sun+18] Roger Sun et al. "A radiomics approach to assess tumour-infiltrating CD8 cells and response to anti-PD-1 or anti-PD-L1 immunotherapy: an imaging biomarker, retrospective multicohort study". In: Lancet Oncol. 19.9 (2018), pp. 1180-1191.

[Sun+20b] Zongqiong Sun et al. Radiomics study for predicting the expression of PD-L1 in non-small cell lung cancer based on CT images and clinicopathologic features. 2020.

[Suo+16] Kathleen C Suozzi et al. "Immune-related sarcoidosis observed in combination ipilimumab and nivolumab therapy". In: JAAD case reports 2.3 (2016), pp. 264-268.

[Tan+19] Xianzheng Tan et al. "Radiomics nomogram outperforms size criteria in discriminating lymph node metastasis in resectable esophageal squamous cell carcinoma". en. In: Eur. Radiol. 29.1 (Jan. 2019), pp. 392-400.

[Tan+18] Chad Tang et al. "Development of an Immune-Pathology Informed Radiomics Model for Non-Small Cell Lung Cancer". en. In: Sci. Rep. 8.1 (Jan. 2018), p. 1922. 
[Tar+16] Francesca Tartari et al. "Economic sustainability of antiPD-1 agents nivolumab and pembrolizumab in cancer patients: Recent insights and future challenges"'. en. In: Cancer Treat. Rev. 48 (July 2016), pp. 20-24.

[Ten+18] Feifei Teng et al. "Progress and challenges of predictive biomarkers of anti PD-1/PD-L1 immunotherapy: A systematic review". en. In: Cancer Lett. 414 (Feb. 2018), pp. 166-173.

[TM20] Rajat Thawani and Syed Atif Mustafa. "The future of radiomics in lung cancer". In: The Lancet Digital Health 2.3 (2020), e103.

[The+00] Patrick Therasse et al. "New Guidelines to Evaluate the Response to Treatment in Solid Tumors". en. In: J. Natl. Cancer Inst. 92.3 (Feb. 2000), pp. 205-216.

[TBS93] S N Thibodeau, G Bren, and D Schaid. "Microsatellite instability in cancer of the proximal colon". en. In: Science 260.5109 (May 1993), pp. 816-819.

[Tie20] Jeanne Tie. "Tailoring immunotherapy with liquid biopsy". In: Nature Cancer 1.9 (Sept. 2020), pp. 857-859.

[Tim+20] Janita E van Timmeren et al. "Radiomics in medical imaging-"how-to" guide and critical reflection". en. In: Insights Imaging 11.1 (Aug. 2020), p. 91.

[Tir+15] Sree Harsha Tirumani et al. "Radiographic Profiling of Immune-Related Adverse Events in Advanced Melanoma Patients Treated with Ipilimumab". en. In: Cancer Immunol Res 3.10 (Oct. 2015), pp. 1185-1192.

[Tre+20] Stefano Trebeschi et al. "Deep learning distinguishing pulmonary progression from pulmonary sarcoid-like lesions in immunotherapy-treated melanoma patients". In: British Journal of Cancer, accepted for publication (2020). 
[Tre+21a] Stefano Trebeschi et al. "Development of a prognostic AImonitor for metastatic urothelial cancer patients receiving immunotherapy". In: Submitted for publication. (2021).

[Tre+19] Stefano Trebeschi et al. "Predicting response to cancer immunotherapy using noninvasive radiomic biomarkers". In: Ann. Oncol. 30.6 (2019), pp. 998-1004.

[Tre+21b] Stefano Trebeschi et al. "Prognostic value of deep learning mediated treatment monitoring in lung cancer patients receiving immunotherapy". In: Frontiers in Oncology, accepted for publication (2021).

[Tun+] Ilke Tunali et al. Hypoxia-related radiomics predict immunotherapy response: A multi-cohort study of NSCLC.

[Tun+19] Ilke Tunali et al. "Novel clinical and radiomic predictors of rapid disease progression phenotypes among lung cancer patients treated with immunotherapy: An early report". In: Lung Cancer 129.January (2019), pp. 75-79.

[TS16] Samra Turajlic and Charles Swanton. "Metastasis as an evolutionary process". en. In: Science 352.6282 (Apr. 2016), pp. 169-175.

[Tur+16] Riku Turkki et al. "Antibody-supervised deep learning for quantification of tumor-infiltrating immune cells in hematoxylin and eosin stained breast cancer samples". en. In: J. Pathol. Inform. 7 (Sept. 2016), p. 38.

[Twy+15] Christina Twyman-Saint Victor et al. "Radiation and dual checkpoint blockade activate non-redundant immune mechanisms in cancer". en. In: Nature 520.7547 (Apr. 2015), pp. 373-377.

[USa] U S Food And. U.S. Food and Drug Administration. Drugs (KEYTRUDA Label). https : / / www . accessdata . fda . gov / drugsatfda _ docs / label / 2017/125514s014lbl . pdf. Accessed: 2018-9-5. 
[U Sb] U S Food And. U.S. Food and Drug Administration. Drugs (OPDIVO Label). https : / / www . accessdata . fda . gov / drugsatfda _ docs / label / 2017 / 125554s0551bl .pdf. Accessed: 2018-9-5.

[Ume+20] Yoshie Umemura et al. DCE-MRI perfusion predicts pseudoprogression in metastatic melanoma treated with immunotherapy. 2020.

[Ung+16] Joseph M Unger et al. "The Role of Clinical Trial Participation in Cancer Research: Barriers, Evidence, and Strategies". en. In: Am Soc Clin Oncol Educ Book 35 (2016), pp. 185-198.

[US ] US Food And Drug Administation. Drug approval package. Bidil (Isosorbide Dinitrate and Hydralazine Hydrochloride) Tablets. https : //www.accessdata.fda.gov/drugsatfda_docs / nda/2005/020727_s000_BidilTOC.cfm. Accessed: 2020-12-17.

[Vai+20] Pranjal Vaidya et al. "Novel, non-invasive imaging approach to identify patients with advanced non-small cell lung cancer at risk of hyperprogressive disease with immune checkpoint blockade". en. In: I Immunother Cancer 8.2 (Oct. 2020).

[Val+15] Martin Vallières et al. A radiomics model from joint FDGPET and MRI texture features for the prediction of lung metastases in soft-tissue sarcomas of the extremities. 2015.

[Val+17] Martin Vallières et al. Data from Head-Neck-PET-CT. 2017.

[Van+15] Sarah J Van Riel et al. "Observer variability for classification of pulmonary nodules on low-dose CT Images and its effect on nodule management1". In: Radiology 277.3 (2015), pp. 863-871. 
[Vee+20] Harini Veeraraghavan et al. "Machine learning-based prediction of microsatellite instability and high tumor mutation burden from contrast-enhanced computed tomography in endometrial cancers". en. In: Sci. Rep. 10.1 (Oct. 2020), p. 17769.

[Ver+18] Vivek Verma et al. "A systematic review of the cost and cost-effectiveness studies of immune checkpoint inhibitors". en. In: J Immunother Cancer 6.1 (Nov. 2018), p. 128.

[VS13] Liza C Villaruz and Mark A Socinski. "The clinical viewpoint: definitions, limitations of RECIST, practical considerations of measurement". en. In: Clin. Cancer Res. 19.10 (May 2013), pp. 2629-2636.

[Vok+18] E E Vokes et al. "Nivolumab versus docetaxel in previously treated advanced non-small-cell lung cancer (CheckMate 017 and CheckMate 057): 3-year update and outcomes in patients with liver metastases". en. In: Ann. Oncol. 29.4 (Apr. 2018), pp. 959-965.

[Voo+17] Khinh Ranh Voong et al. "Beyond PD-L1 testingemerging biomarkers for immunotherapy in non-small cell lung cancer". In: Annals of translational medicine 5.18 (2017).

[Vou20] Ioannis A Voutsadakis. "Prediction of Immune checkpoint inhibitors benefit from routinely measurable peripheral blood parameters". In: Chinese Clinical Oncology 9.2 (2020), pp. 19-19.

[Wan+20] Liansheng Wang et al. A novel approach combined transfer learning and deep learning to predict TMB from histology image. 2020.

[WN11] Yi-Xiang J Wang and Chin K Ng. "The impact of quantitative imaging in medicine and surgery: Charting our course for the future." In: Quant. Imaging Med. Surg. 1.1 (2011), pp. 1-3. 
[Wan+19] Zhijie Wang et al. "Assessment of Blood Tumor Mutational Burden as a Potential Biomarker for Immunotherapy in Patients With Non-Small Cell Lung Cancer With Use of a Next-Generation Sequencing Cancer Gene Panel". en. In: JAMA Oncol 5.5 (May 2019), pp. 696-702.

[Web+15] Jeffrey S Weber et al. "Nivolumab versus chemotherapy in patients with advanced melanoma who progressed after anti-CTLA-4 treatment (CheckMate 037): a randomised, controlled, open-label, phase 3 trial". en. In: Lancet Oncol. 16.4 (Apr. 2015), pp. 375-384.

[Wee+19] Leonard Wee et al. Data from NSCLC-RadiomicsInterobserver1. 2019.

[Wee+98] J C Weeks et al. "Relationship between cancer patients' predictions of prognosis and their treatment preferences". en. In: JAMA 279.21 (June 1998), pp. 1709-1714.

[Wet+02] Stephan G Wetzel et al. "Relative cerebral blood volume measurements in intracranial mass lesions: Interobserver and intraobserver reproducibility study". In: Radiology 224.3 (2002), pp. 797-803.

[Whi08] T L Whiteside. "The tumor microenvironment and its role in promoting tumor growth". en. In: Oncogene 27.45 (Oct. 2008), pp. 5904-5912.

[Wol+15] Jedd D Wolchok et al. "Efficacy and safety results from a phase III trial of nivolumab (NIVO) alone or combined with ipilimumab (IPI) versus IPI alone in treatment-naive patients (pts) with advanced melanoma (MEL) (CheckMate 067)". In: J. Clin. Orthod. 33.18_suppl (June 2015), LBA1-LBA1.

[Wol+09] Jedd D Wolchok et al. "Guidelines for the evaluation of immune therapy activity in solid tumors: immune-related response criteria". en. In: Clin. Cancer Res. 15.23 (Dec. 2009), pp. 7412-7420. 
[Woo19] Marcus Woo. "An AI boost for clinical trials". en. In: $\mathrm{Na}$ ture 573.7775 (Sept. 2019), S100-S102.

[Wu09] Anna M Wu. "Antibodies and antimatter: the resurgence of immuno-PET". en. In: J. Nucl. Med. 50.1 (Jan. 2009), pp. 2-5.

[Wu+19] Wei Wu et al. "Comparison of prediction models with radiological semantic features and radiomics in lung cancer diagnosis of the pulmonary nodules: a case-control study". en. In: Eur. Radiol. 29.11 (Nov. 2019), pp. 61006108.

[WH18] Yuxin Wu and Kaiming He. "Group Normalization: 15th European Conference, Munich, Germany, September 8-14, 2018, Proceedings, Part XIII". In: Computer Vision - ECCV 2018. Ed. by Vittorio Ferrari et al. Vol. 11217. Lecture Notes in Computer Science. Cham: Springer International Publishing, 2018, pp. 3-19.

[Yan+18] Ke Yan et al. "DeepLesion: automated mining of large-scale lesion annotations and universal lesion detection with deep learning". en. In: J Med Imaging (Bellingham) 5.3 (July 2018), p. 036501.

[Yin08] Jennifer G Dy Ying Cui. “Orthogonal Principal Feature Selection". In: (2008).

[Yip+17a] Stephen S F Yip et al. Associations between radiologistdefined semantic and automatically computed radiomic features in non-small cell lung cancer. Tech. rep. 2017.

[Yip+17b] Stephen SF Yip et al. "Associations between radiologistdefined semantic and automatically computed radiomic features in non-small cell lung cancer". In: Scientific reports 7.1 (2017), pp. 1-11.

[Yor+19] Afua A Yorke et al. Pelvic Reference Data [Dataset]. 2019. 
[Yu+19] Y Yu et al. Predictors of Early Response to Immunotherapy in Head and Neck Cancer: A Secondary Clinical and Radiomic Analysis of a Prospective Randomized Trial with Nivolumab. 2019.

[ZF14] Matthew D Zeiler and Rob Fergus. "Visualizing and Understanding Convolutional Networks". In: Computer Vision - ECCV 2014. Springer International Publishing, 2014, pp. 818-833.

[ZWZ17] Pengyue Zhang, Fusheng Wang, and Yefeng Zheng. Self supervised deep representation learning for fine-grained body part recognition. 2017.

[Zha+18] Zijian Zhang et al. “A predictive model for distinguishing radiation necrosis from tumour progression after gamma knife radiosurgery based on radiomic features from MR images". en. In: Eur. Radiol. 28.6 (June 2018), pp. 22552263.

[Zha+19a] Zizhao Zhang et al. "Pathologist-level interpretable whole-slide cancer diagnosis with deep learning". In: Nature Machine Intelligence 1.5 (May 2019), pp. 236-245.

[Zha+19b] Pengfei Zhao et al. "Mismatch repair deficiency/microsatellite instability-high as a predictor for anti-PD-1/PD-L1 immunotherapy efficacy". en. In: J. Hematol. Oncol. 12.1 (May 2019), p. 54.

[Zha+20] Shengyu Zhao et al. "Unsupervised 3D End-to-End Medical Image Registration With Volume Tweening Network". en. In: IEEE J Biomed Health Inform 24.5 (May 2020), pp. 1394-1404.

[Zhe+20] Xueyi Zheng et al. Deep learning radiomics can predict axillary lymph node status in early-stage breast cancer. 2020. 
[Zho+20] Xiaoxiang Zhou et al. "Are immune-related adverse events associated with the efficacy of immune checkpoint inhibitors in patients with cancer? A systematic review and meta-analysis". en. In: BMC Med. 18.1 (Apr. 2020), p. 87.

[Zit+17] Federica Zito Marino et al. "Are tumor-infiltrating lymphocytes protagonists or background actors in patient selection for cancer immunotherapy?" en. In: Expert Opin. Biol. Ther. 17.6 (June 2017), pp. 735-746.

[Zuh+21] Zuhir Bodalal, Stefano Trebeschi et al. "The future of artificial intelligence applied to immunotherapy trials". In: Neoadjuvant Immunotherapy Treatment of Localized Genitourinary Cancers: Multidisciplinary Management. Book chapter accepted for publication. Springer, 2021. 



\section{Valorisation}

\section{Relevance}

Cancer immunotherapy is becoming a standard treatment for advanced-stage cancer patients. While results from numerous clinical trials show significantly higher response and survival rate compared to the standard chemoradiotherapy, there is still a number of patients who do not benefit from starting (or continuing) the treatment. In the meanwhile, patients can experience treatment side-effects, normally manifested as auto-immune and inflammatory disorders, such as dermatitis, pneumonitis, colitis, hepatitis, and granulomatous disease [Zho+20]. While the impact of treatment side-effects on the response is still under investigation, with meta-analyses pointing to them as positive prognostic factors [Zho+20], it is well known that the cost of immune checkpoint inhibitors can pose a significant strain on hospital resources, and public resources in general, especially in Europe where access to healthcare services are guaranteed, regulated and subsidised by the government. Immunotherapy is known to cost up to ten times more than chemotherapeutic options. The average immunotherapeutic treatment costs in the order of the hundreds of thousands of euros, compared to the tens of thousands needed for traditional chemotherapy [Ver+18]. In patients that are not likely to derive any clinical benefit from immunotherapy, these resources could be reallocated for other therapeutic options. In this thesis, we propose the use of artificial intelligence on already-available routine clinical imaging. By enhancing the analytic process with AI, we reach an accurate, and cost-effective marker that, by virtue of being trained on routine imaging, can scale well from academic centers to peripheral hospitals. 


\section{Target population}

This thesis has a wide target population. It includes radiologists who are already using medical imaging as a fundamental tool in their diagnostic process, and who will likely be in charge of the new technologies that will be developed out of this thesis and in the field of artificial intelligence in general. Some of the techniques described and proposed here, such radiomics analysis of cancer lesions (Chapters 2 and 3), or whole-body prognostic monitoring (Chapters 4, 5, and 6), offer a new way to analyze radiological scans. Radiologists will be at the forefront of the implementation (Chapter 7 and 8). Their contribution will be vital in developing the interaction of these technologies within the clinical team.

Radiologists will also play a role when these technologies will enter the multidisciplinary tumor board. As mentioned in Chapters 7 and 9, the envisioned usage of the AI methods developed within this thesis reflects this point. We envision our system to be an interactive window into the status of the patient, their potential risks for survival, and suggestions for most likely response to different treatment options. Treatment planning will be defined according to this data and the input of the clinicians in the board. Members of the tumour board will be essential in the study of the interaction between AI and clinicians, and the development of a coordinated approach for its development and implementation.

In this, tech companies, specifically the ones that are involved in the development of AI-based healthcare solutions will also play a role. These entities possess the know-how of the actual implementation process, which should cover multiple practical aspects, from the hardware requirement to the steps for approval from the healthcare regulators (e.g. the European Medicines Agency [Coh+20]).

The methodology presented in this thesis targets mostly cancer patients with advanced disease, namely spread outside of the original location and throughout the body. In the majority of the cases, the 
main clinical intent in these patients is palliative, in other words, to improve quality of life more than survival. Even in immunotherapy, where we observed higher survival rates and even some complete responses, palliative intent remains the primary focus [SC18]. AI-based technologies, such as the one presented, aside from suggesting the most effective treatment which would in turn improve response and overall survival, could also help pin-point localized conditions of risk, which could impair quality of life, and give the clinician indications to address the issue. In summary, the overall expected outcome from employing AI-based evidence decision-making is improved survival and improved quality of life.

To the scientific community, we provide evidence not only of the efficacy of AI-mediated pipelines, but also the success of multidisciplinarity in research, with all the work published in this thesis relying on the joint, coordinated work of experts from different fields.

\section{Innovation and future}

The results of this thesis show how prognostic and predictive factors can be found by artificial intelligence-based image analysis on routine radiological scans of cancer patients receiving immunotherapy. We filled the knowledge gap, and demonstrated a link between imagingderived morphological features to its biological profile and treatment outcome in immunotherapy, being one of the first investigations to do so. We further identified the limitation of the classical image analysis pipeline that we employed in the initial study, which involves a radiologist identifying a region of interest to analyse, and improved it to a new analytic pipeline, termed prognostic monitoring, which does not require manual input, which is not limited to static baseline imaging, and which utilises the entire whole-body scan. This represents a shift from the image analysis pipelines that have been proposed so far, not for its full automatic aspect, but rather because we shift the identification of factors to the AI, removing any bias that may come from 
human analytics. In other words, we are not developing a pipeline which automates the manual work already performed in the clinics, but rather developing something that was not even present in the clinics, i.e. tracking of all morphological changes in the body. We are not employing AI to reduce the information included in a whole-body scan to a limited set of factors easily understandable by humans, such as change in tumor size, but we rather let the AI-algorithm run independently to determine what are the factors that correlate with worse prognosis. We are not training an AI-model to imitate us, or think like us (i.e. smaller tumor, better outcomes), rather we let the AI-algorithm draw its own conclusions from the data.

We envision more studies following this idea, as it would produce novel solutions, which are complementary to, and not disruptive of, the current clinical world. The AI-algorithms developed in this study are still prototypes. Future propostive will be to extend them to a fully functional medical device, tested on pan-cancer and pan-treatment clinical trials, and approved by the appropriate regulatory agencies. 


\section{Summary}

Chapter 2. Lesion response prediction to immunotherapy.

In this chapter, we aimed to link imaging-derived, radiomics features with outcome, lesion-wise. Our findings suggest associations between radiomics features and immunotherapy response. Lesions that are more likely to respond to immunotherapy typically present with more heterogeneous morphological profiles with non-uniform density patterns and compact borders. Moreover, a machine learning model is provided that could be used within the context of lesion response to treatment, patient treatment response, and response pattern characterization.

Chapter 3. Lesion diagnosis to therapy-induced lung disease.

Aim of this chapter was to apply artificial intelligence analytic pipelines on routine medical imaging for the diagnosis of sarcoid-like granulomatous lesions induced by novel cancer immunotherapeutic agents. We found significant performance in the diagnosis of sarcoid-like granulomatous lesions, while simultaneously significantly improving the performance of the original screening network for the diagnosis of pulmonary metastases. Moreover, the network was able to distinguish between sarcoid-like granulomatous lesions and non-specific post-infection granulomas. Diagnostic signatures were also found to possess prognostic relevance.

Chapter 4. Prognostic value of chest-imaging monitoring.

In this chapter, we aimed to investigate the potential prognostic value of AI-mediated monitoring in NSCLC patients receiving PD-1 blockade. We hypothesized the existence of quantitative imaging features describing a set of gross morphological changes happening during treatment that hold prognostic information. Based on image-to-image registration, we develop a deep learning algorithm for the detection 
of changes between serial imaging of the same patient. Our results demonstrate the existence of such factors (as described by the AI on imaging), that are tumor-related, such as nodal, lung and bone lesions, as well as non-tumor related, such as pleural effusions, atelectasis and non-specific consolidations.

Chapter 5. Whole-body imaging-based prognostic monitoring.

In this chapter, we investigated the prognostic information of AI-derived whole-body imaging monitoring markers in advanced urothelial cancer receiving checkpoint inhibitors. We hypothesised that quantitative AI-derived features describing morphological changes happening during the course of treatment could hold prognostic information. To this end, we designed and implemented a prognostic AI-monitor (PAM), based on the prototype design of Chapter 4, and extended to handle heterogeneous datasets and abdominal imaging. Our findings demonstrate that PAM is complementary to existing monitoring methods, while reaching comparable or superior accuracy. We argue that this could be the result of PAM's ability to analyze the whole body, including non-target cancer lesions and non-cancer lesions.

Chapter 6. Prognostic response patterns in brain imaging.

In this chapter, we present an expansion of the PAM analytic pipeline to brain imaging of BM patients receiving immunotherapy. Our results demonstrate that PAM can be extended to imaging modalities beyond $\mathrm{CT}$, and be used to capture prognostic response patterns that are unique and complementary to a wide range of different brain-specific markers, currently used in the clinics.

Chapter 7. The future of artificial intelligence immunotherapy trials.

Clinical trials serve as a barrier of entry for new interventions and treatments prior to implementation in routine clinical practice. At its essence, the primary role of a clinical trial is to monitor a patient longitudinally using the diagnostic disciplines (radiology, pathology, and laboratory medicine) to assess clinical outcomes. As the diagnostic 
fields have begun to fully digitalise, large volumes of data are being generated per patient - creating a ripe environment for the implementation of AI. In this chapter, we will explore how AI has been applied in each of these diagnostic disciplines and discuss how this may influence clinical trials in the future.

Chapter 8. Towards integrated healthcare.

Medical imaging is a vital part of the clinical decision making process, especially in an oncological setting. Radiology has experienced a great wave of change and the advent of quantitative imaging has provided a unique opportunity to analyze patient images objectively. Leveraging AI, there is increased potential for synergy between physicians and computer networks - via computer aided diagnosis (CAD), computer aided prediction of response (CARP), and computer aided biological profiling (CABP). The ongoing digitalization of other specialties further opens the door for even greater multidisciplinary integration. In this chapter, we envision the development of an integrated system composed of an aggregation of sub-systems interoperating with the aim of achieving an overarching functionality (in this case better CAD, CARP, and CABP). This will require close multidisciplinary cooperation between the clinicians, biomedical scientists, and (bio)engineers as well as an administrative framework where the departments will operate not in isolation but in successful harmony. 



\section{Acknowledgments}

Firstly, I would like to express my sincere gratitude to my protomors, Prof. Regina Beets-Tan and Prof. Hugo Aerts, for their continuous support of my $\mathrm{PhD}$ research, for their time, patience, motivation, and for all knowledge I have acquired from them in the last five years. Their guidance helped me in the time of research and writing of this thesis, as well as in the planning and development of my own research line, which I will continue pursuing after my PhD. Their mentorship helped me to become an independent scientist.

Besides my protomots, I would like to thank the rest of my thesis committee. This includes the members of the assessment committee, Prof. Dirk de Ruysscher, Prof. Paul Hofman, Prof. Paul Baas, Prof. Klaus Maier-Hein and Dr. Peters, as well as the PhD assessment committee from the Oncology Graduate School of Amsterdam, Prof. Uulke van der Heide, Prof. Koen Hartemink, and Dr. Wouter Vogel. Their insightful comments and availability during my $\mathrm{PhD}$ trajectory allowed me to strengthen my thesis, and my research in general.

My sincere thanks also goes to the oncologists we have had the pleasure and honor to work with in the last five years, Prof. John Haanen, Prof. Christian Blank, Prof. Egbert Smit, and Prof. Michiel van der Heijden, and their collaborators and lab members, and our own clinicians within the department of radiology, the radiologists, and the technicians, for their immense help, support, and guidance.

I would also like to extend a word of gratitude to our Swiss and American collaborators and co-workers from the University Hospital of Zurich and the Harvard Medical School, with whom we have instantiated a solid and fruitful collaboration, which I'm sure will be strengthened in the years to come.

I thank my fellow lab mates and colleagues from the department of Radiology, and the immunotherapy imaging team, who surrounded 
me in the last half decade. Thank you for your company, advice, help and support, making every day at the office a pleasant experience. I could have not asked for better colleagues. A special thank to my direct colleagues of the immunotherapy team Dan Linh, Zuhir, and Teresa for the time taken in answering all my questions about radiology, immunology, oncology, and biology, for the brainstorming on our revolutionary ideas, the late hours working, and the laughter. I could have not made it without you.

Last but not least, I would like to thank my friends here in Amsterdam, who made my stay so far one of the best experiences of my life, and who enabled me to discover myself, and to grow. I would like to extend my gratitude to friends, teachers, and co-workers I left in Germany and Italy. I would like to thank my family, especially my sister, and their support during my childhood and (rebellious) adolescence.

To all the amazing, diverse, and crazy people in my life, thank you. 


\section{Published work}

Stefano Trebeschi et al. "Predicting response to cancer immunotherapy using noninvasive radiomic biomarkers". In: Ann. Oncol. 30.6 (2019), pp. 998-1004

Stefano Trebeschi et al. "Deep learning distinguishing pulmonary progression from pulmonary sarcoid-like lesions in immunotherapytreated melanoma patients". In: British Journal of Cancer, accepted for publication (2020)

Stefano Trebeschi et al. "Prognostic value of deep learning mediated treatment monitoring in lung cancer patients receiving immunotherapy". In: Frontiers in Oncology, accepted for publication (2021)

Stefano Trebeschi et al. "Development of a prognostic AI-monitor for metastatic urothelial cancer patients receiving immunotherapy". In: Submitted for publication. (2021)

Stefano Trebeschi, Thi Dan Linh Nguyen-Kim et al. "AI-driven identification of prognostic response patterns to immunotherapy of melanoma brain metastases". In: Submitted for publication. (2021)

Zuhir Bodalal, Stefano Trebeschi et al. "The future of artificial intelligence applied to immunotherapy trials". In: Neoadjuvant Immunotherapy Treatment of Localized Genitourinary Cancers: Multidisciplinary Management. Book chapter accepted for publication. Springer, 2021

Zuhir Bodalal, Stefano Trebeschi, and Regina Beets-Tan. "Radiomics: a critical step towards integrated healthcare". In: Insights into imaging 9.6 (2018), pp. 911-914 



\section{About the author}

Stefano Trebeschi was born on the 20th of June, 1991, in Desenzano del Garda, Italy. He grew up in Cavriana, Italy. At the age of 19, he moved to South Tyrol, where he started his bachelor degree in computer science and engineering at the Free University of Bolzano-Bozen. During this period, he got involved in the research on software engireering, within the Department of Computer Science. He graduated in 2013, supervised by Dr. Ilenia Fronza and Dr. Andrea Janes, with a thesis on data analytics for software development monitoring.

In 2013 he moved to Munich, Germany, to start his master in computer science at the Technical University of Munich. As part of his master program, he got involved in a series of projects in medical imaging, and machine learning, both within the Department of Computer Science, as well as the Department of Neurology at the Klinikum Rechts der Isar. Here he graduated in 2016, under the supervision of Dr. Alexander Valentinitsch, Prof. dr. Jan Kirschke (geb. Bauer), and Prof. dr. Bjoern Menze, for the identification of osteoporosis patients at risk of vertebral fracture. He moved to Amsterdam, the Netherlands, on the same year to start his PhD at the Netherlands Cancer Institute, under the supervision of Prof. dr. Regina Beets-Tan and Prof. Hugo Aerts, from the Harvard Medical School.

During his $\mathrm{PhD}$, Stefano has focused his research line in imaging of cancer patients receving immunotherapy, on which he has authored six papers, a book chapter, and talks at the European Society of Radiology (ESR), European Hematology Association (EHA), and European Society of Medical Imaging Informatics (EUSOMII). In these five years, he has supervised internships and theses from master students of the University of Twente, among other national and international coollaborations. 